\title{
Straight-Line Rectangular Drawings of Clustered Graphs
}

\author{
Patrizio Angelini • Fabrizio Frati • \\ Michael Kaufmann
}

Received: 15 August 2009 / Revised: 19 May 2010 / Accepted: 13 September 2010 /

Published online: 5 October 2010

(C) Springer Science+Business Media, LLC 2010

\begin{abstract}
We show that every $c$-planar clustered graph has a straight-line $c$-planar drawing in which each cluster is represented by an axis-parallel rectangle, thus solving a problem posed by Eades, Feng, Lin, and Nagamochi (Algorithmica 44(1):1-32, 2006).
\end{abstract}

Keywords Clustered graphs $\cdot$ Planar graphs $\cdot$ Straight-line drawings $\cdot$ Rectangles

\section{Introduction}

A clustered graph is a pair $C(G, T)$, where $G$ is a graph, called underlying graph, and $T$ is a rooted tree, called inclusion tree, such that the leaves of $T$ are the vertices of $G$. Each internal node $v$ of $T$ corresponds to the subset of vertices of $G$, called cluster, that are the leaves of the subtree of $T$ rooted at $v$.

Clustered graphs are widely used in applications where it is needed at the same time to represent relationships between entities and to group entities with semantic affinities. For example, in the Internet network, links among routers give rise to a graph; geographically close routers are grouped into areas, which in turn are grouped into Autonomous Systems.

Visualizing clustered graphs turns out to be a difficult problem, due to the simultaneous need for a readable drawing of the underlying structure and for a good

P. Angelini · F. Frati $(\bowtie)$

Departimento di Informatica e Automazione, Roma Tre University, Rome, Italy

e-mail: frati@dia.uniroma3.it

P. Angelini

e-mail: angelini@dia.uniroma3.it

M. Kaufmann

Wilhelm-Schickard-Institut für Informatik, Universität Tübingen, Tübingen, Germany

e-mail: mk@informatik.uni-tuebingen.de 
rendering of the recursive clustering relationship. As for the visualization of graphs, the most important aesthetic criterion for a drawing of a clustered graph to be "nice" is commonly regarded to be the planarity, which however needs a refinement in the contest of clustered graphs, in order to deal also with the clustering structure.

A drawing $\Gamma$ of a clustered graph $C(G, T)$ consists of a drawing of $G$ (each vertex is a point in the plane and each edge is as Jordan curve between its end-vertices) and of a representation of each node $\mu$ of $T$ as a simple closed region containing all and only the vertices that belong to $\mu$. A drawing is $c$-planar if it has no edge crossings (i.e., the drawing of the underlying graph is planar), no edge-region crossings (i.e., an edge intersects the boundary of a cluster at most once), and no region-region crossings (i.e., no two cluster boundaries cross).

Given a clustered graph, testing whether it admits a $c$-planar drawing is a problem of unknown complexity, perhaps the most studied problem in the Graph Drawing community during the last ten years [1-5, 8, 15, 17-22].

Suppose that a $c$-planar clustered graph $C$ is given together with a $c$-planar embedding, that is, together with an equivalence class of $c$-planar drawings of $C$, where two $c$-planar drawings are equivalent if they have the same order of the edges incident to each vertex and the same order of the edges incident to each cluster. How can the graph be drawn? Such a problem has been intensively studied in the literature and a number of papers have been presented for constructing $c$-planar drawings of $c$-planar clustered graphs within many drawing conventions.

Eades et al. show in [12] how to construct $O\left(n^{2}\right)$-area $c$-planar orthogonal drawings and $O\left(n^{2}\right)$-area $c$-planar poly-line drawings of $c$-planar clustered graphs with clusters drawn as axis-parallel rectangles. Di Battista et al. [6] show algorithms and bounds for constructing small-area drawings of $c$-planar clustered trees within several drawing styles. The strongest result in the area is perhaps the one that Eades et al. present in [11]. Namely, the authors show an algorithm for constructing $c$-planar straight-line drawings of $c$-planar clustered graphs in which each cluster is drawn as a convex region (see also a paper of Nagamochi and Kuroya [25]). Such an algorithm requires, in general, exponential area. However, in [16] Feng et al. have shown that such a bound is asymptotically optimal in the worst case.

In this paper we address a problem posed by Eades et al. in [11, 13, 15], namely whether every $c$-planar clustered graph has a straight-line rectangular drawing, i.e., a $c$-planar straight-line drawing in which each cluster is drawn as an axis-parallel rectangle. An example of a straight-line rectangular drawing of a $c$-planar clustered graph is given in Fig. 1. Eades et al. observe how pleasant and readable straight-line rectangular drawings are; however, they provide evidence that their algorithm [11] for constructing $c$-planar straight-line convex drawings of clustered graphs cannot be modified in order to get rectangular clusters without introducing edge-region crossings.

We show that every $c$-planar clustered graph has a straight-line rectangular drawing. We obtain such a result as a corollary of a stronger theorem stating that a straightline rectangular drawing of a $c$-planar clustered graph exists for an arbitrary convexseparated drawing of its outer face, that is, a drawing satisfying some properties of convexity and of visibility among vertices and clusters.

Such a stronger result is proved by means of an inductive algorithm reminiscent of Fáry's drawing algorithm for planar graphs [14]. Namely, the algorithm consists 
Fig. 1 A straight-line rectangular drawing of a $c$-planar clustered graph

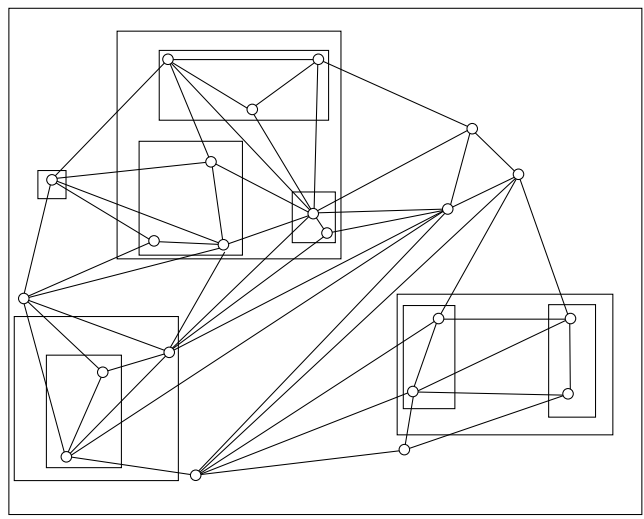

of three inductive cases. Each case considers a clustered graph $C$ and performs an operation (removal of a cluster, split of the graph in correspondence of a separating 3 -cycle, or contraction of an edge) turning $C$ into a smaller clustered graph $C^{\prime}$ for which a straight-line rectangular drawing can be inductively constructed. Then, such a drawing can be easily augmented to a straight-line rectangular drawing of $C$.

When none of the three inductive cases applies, every cluster contains a vertex incident to the outer face. We call outerclustered graph a clustered graph satisfying this property. We prove that every outerclustered graph admits a straight-line rectangular drawing even if a convex-separated drawing of its outer face is arbitrarily fixed, thus providing a base case for the above inductive algorithm for general clustered graphs. In order to draw an outerclustered graph $C$, we split it into three linearly-ordered outerclustered graphs (an even more restricted family of clustered graphs), we separately draw such graphs, and we compose the obtained drawings to get a drawing of $C$.

A linearly-ordered outerclustered graph $C(G, T)$ is an outerclustered graph in which the inclusion tree $T$ is such that removing its leaves (corresponding to the vertices of $G$ ) and their incident edges yields a path. A drawing algorithm is provided for constructing a straight-line rectangular drawing of any linearly-ordered outerclustered graph $C(G, T)$ for an arbitrary convex-separated drawing of its outer face. Such an inductive algorithm splits $C(G, T)$ into smaller linearly-ordered outerclustered graphs and combines the inductively-constructed drawings of such subgraphs to obtain a drawing of $C(G, T)$.

The rest of the paper is organized as follows. In Sect. 2 we introduce some preliminaries and definitions; in Sect. 3 we show a drawing algorithm for linearly-ordered outerclustered graphs; in Sect. 4 we show a drawing algorithm for outerclustered graphs; in Sect. 5 we show a drawing algorithm for general clustered graphs; finally, in Sect. 6 we conclude and present some open problems.

\section{Preliminaries}

We assume familiarity with graphs, clustered graphs and their drawings (see, e.g., $[7,24])$. 
A graph $G$ is $k$-connected if removing any $k-1$ vertices leaves $G$ connected; 3 -connected and 2-connected graphs are also called triconnected and biconnected graphs, respectively.

Let $C(G, T)$ be a clustered graph. An edge $(u, v)$ of $G$ is incident to a cluster $\mu$ of $T$ if $u$ belongs to $\mu$ and $v$ does not belong to $\mu$. We denote by $\sigma\left(u_{1}, u_{2}, \ldots, u_{k}\right)$ the smallest cluster of $T$ containing vertices $u_{1}, u_{2}, \ldots, u_{k}$ of $G$, i.e., the node of $T$ containing all vertices $u_{1}, u_{2}, \ldots, u_{k}$ and such that none of its children in $T$, if any, contains all vertices $u_{1}, u_{2}, \ldots, u_{k}$. A cluster is minimal if it contains no other clusters. A cluster $\mu$ is an ancestor (descendant) of a cluster $v$ if $\mu$ is an ancestor (descendant) of $v$ in the inclusion tree $T$. A clustered graph $C(G, T)$ is $c$-connected if each cluster induces a connected subgraph of $G$.

Throughout the paper, we allow a clustered graph $C(G, T)$ to have several clusters containing exactly the same set of vertices of $G$. Then, a set of clusters all containing the same set of vertices of $G$ induces a path in $T$.

A drawing $\Gamma$ of a clustered graph $C(G, T)$ consists of a drawing of $G$ (each vertex is represented by a point in the plane and each edge is represented by a Jordan curve between its end-vertices) and of a representation of each node $\mu$ of $T$ as a simple closed region that contains all and only the vertices that belong to $\mu$. If two clusters $\mu$ and $\nu$ contain the same set of vertices and $\nu$ is an ancestor of $\mu$, then the region representing $v$ encloses the region representing $\mu$. In the following, when we say "cluster", we refer both to the set of vertices contained in the cluster and to the region representing the cluster in a drawing, the meaning of the word being clear from the context. A drawing $\Gamma$ has an edge crossing if two edges of $G$ cross; $\Gamma$ has an edge-region crossing if an edge crosses the boundary of a cluster more than once; $\Gamma$ has a region-region crossing if the boundaries of two clusters cross. A drawing of a clustered graph is $c$-planar if it does not have edge crossings, edge-region crossings, and region-region crossings. A graph is $c$-planar if it has a $c$-planar drawing. In this paper we are interested in straight-line rectangular drawings of clustered graphs, i.e., $c$-planar drawings such that each edge is represented by a straight-line segment and each cluster is represented by an axis-parallel rectangle. From now on, even when not explicitly specified, "clustered graph" will always mean $c$-planar clustered graph, while "drawing" will always mean straight-line rectangular drawing.

A clustered graph $C(G, T)$ is maximal if $G$ is a maximal planar graph. In order to prove that every clustered graph admits a straight-line rectangular drawing, it suffices to prove that every maximal clustered graph admits a straight-line rectangular drawing. In fact, every non-maximal $c$-planar clustered graph $C(G, T)$ can be augmented in linear time to a maximal $c$-planar clustered graph $C^{\prime}\left(G^{\prime}, T\right)$ by adding dummy edges to $G$ [23]. Notice that every maximal $c$-planar clustered graph is $c$-connected. Namely, Feng et al. proved in [17] that a clustered graph $C(G, T)$ is $c$-planar if and only if edges can be added to $G$ so that the resulting clustered graph $C^{\prime}\left(G^{\prime}, T\right)$ is $c$-planar and $c$-connected. As no edge can be added to a maximal $c$-planar clustered graph without losing $c$-planarity, every maximal $c$-planar clustered graph is $c$-connected. From now on, we will always assume that the embedding (that is, the order of the edges incident to each vertex) and the outer face of any considered graph $G$ is fixed in advance (hence, $G$ has to be meant as a plane graph). We denote by $o(G)$ the outer face of $G$. A clustered graph $C(G, T)$ is internally-triangulated if 


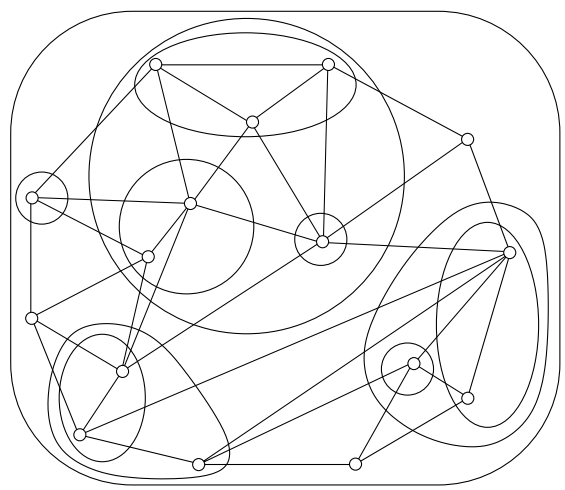

(a)

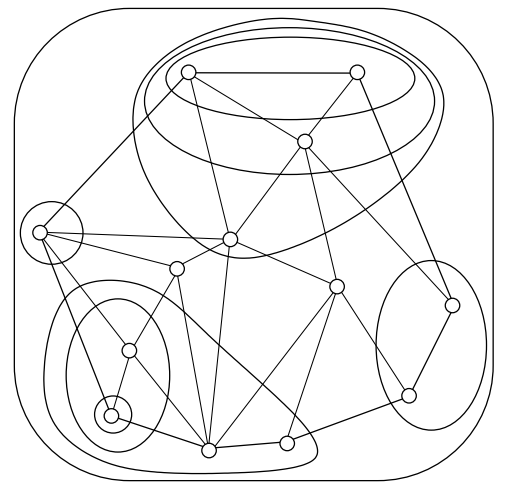

(b)

Fig. 2 (a) A biconnected internally-triangulated clustered graph. (b) A biconnected internally-triangulated outerclustered graph

every internal face of $G$ is delimited by a 3-cycle. Figure 2(a) shows a biconnected internally-triangulated clustered graph.

Let $C(G, T)$ be a clustered graph. Let $f$ be any face of $G$. Denote by $C_{f}\left(G_{f}, T_{f}\right)$ the clustered graph whose underlying graph $G_{f}$ is the cycle induced by the vertices incident to $f$ and whose inclusion tree $T_{f}$ is the subtree of $T$ induced by the clusters containing vertices incident to $f$. In particular, the outer face of $C(G, T)$ is the clustered graph $C_{o(G)}\left(G_{o(G)}, T_{o(G)}\right)$, that is simply denoted by $C_{o}$ in the following. In Sects. 3, 4, and 5, we will prove that a drawing of a clustered graph can be constructed for an arbitrary drawing of its outer face that satisfies some geometric properties to be described below. Then, we say that a straight-line rectangular drawing $\Gamma(C)$ of $C$ completes a straight-line rectangular drawing $\Gamma\left(C_{o}\right)$ of $C_{o}$ if the part of $\Gamma(C)$ representing $C_{o}$ coincides with $\Gamma\left(C_{o}\right)$.

In order to prove that every clustered graph admits a straight-line rectangular drawing, it will be useful to study the class of clustered graphs that is defined below (see Fig. 2(b)).

Definition 1 A $c$-planar clustered graph $C(G, T)$ is an outerclustered graph if:

- O1: every cluster contains at least one vertex incident to $o(G)$;

- O2: the boundary of every cluster $\mu$ that does not contain all the vertices incident to $o(G)$ intersects $o(G)$ exactly twice, that is, it intersects exactly two edges $e_{1}(\mu)$ and $e_{2}(\mu)$ incident to $o(G)$; and

- O3: every edge $(u, v)$ with $\sigma(u)=\sigma(v)$ is incident to $o(G)$.

Throughout the paper, for every cluster $\mu$ of a clustered graph $C(G, T)$ such that the boundary of $\mu$ intersects $o(G)$ exactly twice, we denote by $e_{1}(\mu)$ and $e_{2}(\mu)$ the edges incident to $o(G)$ that are intersected by the boundary of $\mu$.

A maximal outerclustered graph is an outerclustered graph $C(G, T)$ such that $G$ is a maximal plane graph. Observe that the outer face of the underlying graph of a maximal clustered graph is delimited by a 3-cycle. 
Let $C(G, T)$ be a biconnected internally-triangulated outerclustered graph and let $\mathcal{C}$ be any simple cycle in $G$ such that the boundary of every cluster in $T$ containing some but not all the vertices of $\mathcal{C}$ intersects $\mathcal{C}$ exactly twice. Denote by $C^{\prime}\left(G^{\prime}, T^{\prime}\right)$ the clustered graph such that $G^{\prime}$ is the subgraph of $G$ induced by the vertices incident to and internal to $\mathcal{C}$, and such that $T^{\prime}$ is the subtree of $T$ induced by the clusters containing vertices of $G^{\prime}$.

Lemma $1 C^{\prime}\left(G^{\prime}, T^{\prime}\right)$ is a biconnected internally-triangulated outerclustered graph.

Proof Since $G$ is biconnected and internally-triangulated, $G^{\prime}$ is biconnected and internally-triangulated, as well. We prove that $C^{\prime}$ satisfies Property O1 of Definition 1. Suppose that there exists a cluster $\mu$ in $T^{\prime}$ that does not contain any vertex incident to $o\left(G^{\prime}\right)$. Then, $\mu$ contains a vertex internal to $G^{\prime}$, otherwise it would not be a cluster in $T^{\prime}$. Also, $\mu$ contains a vertex incident to $o(G)$, otherwise $C$ would not be an outerclustered graph. Since the boundary of $\mu$ is a closed curve containing a vertex inside $\mathcal{C}$ and a vertex outside $\mathcal{C}$, then either $\mu$ contains a vertex of $\mathcal{C}$ or it intersects twice the same edge of $\mathcal{C}$, in both cases contradicting the $c$-planarity of $C$. Clustered graph $C^{\prime}$ satisfies Property $\mathrm{O} 2$ by hypothesis. We prove that $C^{\prime}$ satisfies Property O3. Suppose that there exists an edge $\left(u^{\prime}, v^{\prime}\right)$ such that $\sigma\left(u^{\prime}\right)=\sigma\left(v^{\prime}\right)$ and $u^{\prime}$ is an internal vertex of $G^{\prime}$. Then, $u^{\prime}$ is an internal vertex of $G$, as well, and $C$ is not an outerclustered graph, a contradiction.

An interesting subclass of the outerclustered graphs is considered in the following (see Fig. 3).

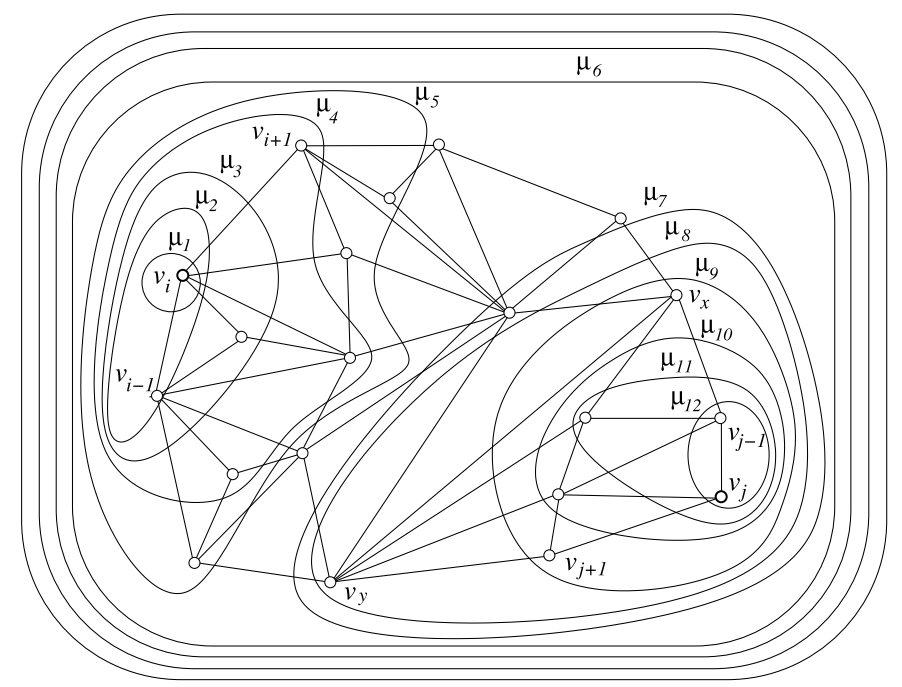

Fig. 3 An outerclustered graph that is linearly-ordered according to the cluster sequence $\mu_{1}, \mu_{2}, \ldots, \mu_{12}$. Notice that $h=6$ 
Definition 2 A biconnected internally-triangulated outerclustered graph $C(G, T)$ is linearly-ordered if there exists a sequence $\mu_{1}, \mu_{2}, \ldots, \mu_{k}$ of clusters in $T$ and an index $1 \leq h \leq k$, such that:

- LO1: for each vertex $v_{j}$ of $G, \sigma\left(v_{j}\right)=\mu_{i}$, for some $1 \leq i \leq k$;

- LO2: let $v_{i}$ and $v_{j}$ be any two vertices incident to $o(G)$ such that $\sigma\left(v_{i}\right)=$ $\mu_{1}$ and $\sigma\left(v_{j}\right)=\mu_{k}$; then $o(G)$ is delimited by two monotone paths $\mathcal{P}_{1}=$ $\left(v_{i}, v_{i+1}, \ldots, v_{j-1}, v_{j}\right)$ and $\mathcal{P}_{2}=\left(v_{i}, v_{i-1}, \ldots, v_{j+1}, v_{j}\right)$, i.e., paths such that, if $\sigma\left(v_{t}\right)=\mu_{a}$ and $\sigma\left(v_{t+1}\right)=\mu_{b}$, then $a \leq b$ if $\left(v_{t}, v_{t+1}\right) \in \mathcal{P}_{1}$ and $b \leq a$ if $\left(v_{t}, v_{t+1}\right) \in \mathcal{P}_{2} ;$ and

- LO3: $\mu_{i+1}$ is the parent of $\mu_{i}$, for each $1 \leq i<h$, and $\mu_{i+1}$ is a child of $\mu_{i}$, for each $h \leq i<k$.

Let $C(G, T)$ be a biconnected internally-triangulated outerclustered graph and suppose that $C$ is linearly-ordered according to a sequence $\mu_{1}, \mu_{2}, \ldots, \mu_{k}$ of clusters of $T$. Let $v_{i} \in \mu_{1}$ and $v_{j} \in \mu_{k}$. Then, denote by $V_{1}$ (resp. by $V_{2}$ ) the vertex set containing all the internal vertices of $\mathcal{P}_{1}$ (resp. of $\mathcal{P}_{2}$ ).

In Sect. 3 we will prove that a drawing of any linearly-ordered outerclustered graph $C(G, T)$ can be obtained even if the drawing of $C_{o}$ is arbitrarily fixed. However, such a drawing of $C_{o}$ will be assumed to be convex-separated, i.e., the drawing of $C_{o}$ satisfies certain geometric properties, as the one that the polygon representing $o(G)$ is convex and as the one that, for every cluster $\mu \in T$, there exists a convex region $R(\mu, v)$ "separating" the vertices belonging to a certain cluster $v$ from the vertices not belonging to such a cluster (in the notation $R(\mu, \nu), \mu$ is the parent of $v$ ). Convex-separated drawings are formally defined below (see Fig. 4).

Definition 3 A straight-line rectangular drawing $\Gamma\left(C_{o}\right)$ of $C_{o}$ is a convex-separated drawing if:

- CS1: the polygon $P$ representing $o(G)$ is convex;

Fig. 4 A convex-separated drawing of the outer face of a linearly-ordered outerclustered graph. In this and other pictures of the paper, the polygonal lines composed of horizontal and vertical segments represent parts of the boundaries of the rectangular clusters

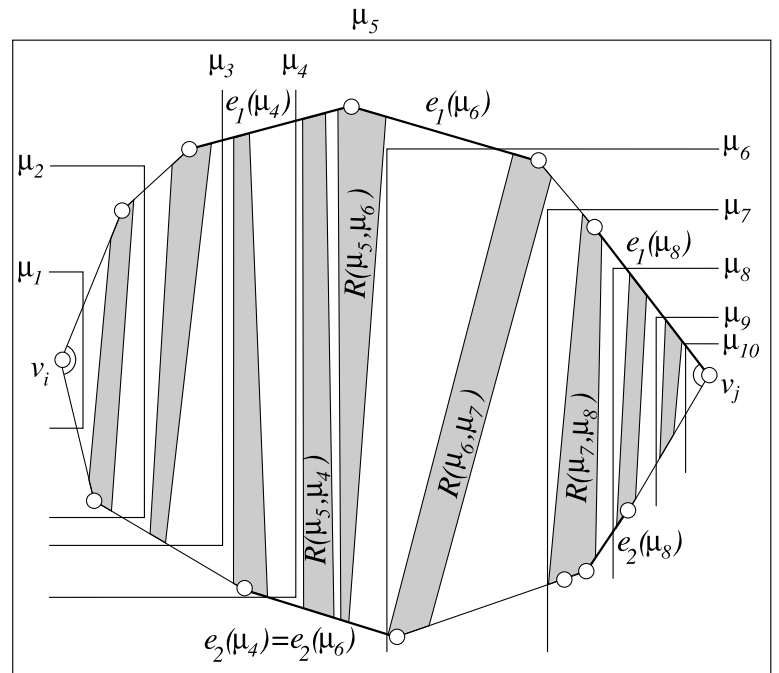


- CS2: there exist two vertices $v_{i}$ and $v_{j}$, with $\sigma\left(v_{i}\right)=\mu_{1}$ and $\sigma\left(v_{j}\right)=\mu_{k}$, such that the angle of $P$ incident to $v_{i}$ and the angle of $P$ incident to $v_{j}$ are strictly less than $180^{\circ}$; and

- CS3: for every pair of clusters $\mu$ and $v$ such that $\mu$ is the parent of $v$ in $T$ and such that $\mu$ is not an ancestor of the smallest cluster containing all the vertices of $o(G)$, there exists a convex region $R(\mu, v)$ such that: (i) $R(\mu, v)$ is entirely contained inside $\mu \cap(P \cup \operatorname{int}(P))$, where $\operatorname{int}(P)$ denotes the interior of polygon P; (ii) for any cluster $\mu^{\prime} \neq \mu$ and any child $\nu^{\prime}$ of $\mu^{\prime}, R(\mu, v)$ intersects neither $R\left(\mu^{\prime}, v^{\prime}\right)$ nor the boundary of $\mu^{\prime}$; (iii) $R(\mu, v) \cap P$ consists of two polygonal lines $l_{1}(\mu, v)$ and $l_{2}(\mu, \nu)$ such that $l_{1}(\mu, \nu)$ belongs to the polygonal line representing $\mathcal{P}_{1}$ in $\Gamma\left(C_{o}\right)$ and $l_{2}(\mu, v)$ belongs to the polygonal line representing $\mathcal{P}_{2}$ in $\Gamma\left(C_{o}\right)$; further, at least one endpoint of $l_{1}(\mu, v)$ (resp. of $l_{2}(\mu, v)$ ) lies on $e_{1}(v)$ (resp. on $e_{2}(v)$ ).

Let $C(G, T)$ be an outerclustered graph with outer face $o(G)$ delimited by a cycle $\mathcal{C}=\left(v_{i}, v_{i+1}, \ldots, v_{j-1}, v_{j}, v_{j+1}, \ldots, v_{i-1}, v_{i}\right)$. Suppose that $C$ is linearly-ordered according to a sequence $\Sigma=\mu_{1}, \mu_{2}, \ldots, \mu_{k}$ of clusters in $T$. Let $\left(v_{x}, v_{y}\right)$ be a chord of $\mathcal{C}$. Consider the clustered graphs $C^{1}\left(G^{1}, T^{1}\right)$ and $C^{2}\left(G^{2}, T^{2}\right)$ such that $G^{1}$ (resp. $G^{2}$ ) is the subgraph of $G$ induced by the vertices incident to and internal to cycle $\mathcal{C}^{1}=\left(v_{x}, v_{x+1}, \ldots, v_{y-1}, v_{y}, v_{x}\right)$ (resp. to cycle $\left.\mathcal{C}^{2}=\left(v_{y}, v_{y+1}, \ldots, v_{x-1}, v_{x}, v_{y}\right)\right)$, and such that $T^{1}$ (resp. $T^{2}$ ) is the subtree of $T$ induced by the clusters containing vertices of $G^{1}$ (resp. of $G^{2}$ ).

Lemma $2 C^{1}\left(G^{1}, T^{1}\right)$ and $C^{2}\left(G^{2}, T^{2}\right)$ are linearly-ordered outerclustered graphs.

Proof We prove the statement for $C^{1}$, the proof for $C^{2}$ being analogous. Refer to Fig. 5.

We claim that $C^{1}$ is a biconnected internally-triangulated outerclustered graph. In order to prove the claim, by Lemma 1 , it suffices to show that no cluster in $T^{1}$ exists that contains some but not all the vertices of $\mathcal{C}^{1}$ and that does not intersect $\mathcal{C}^{1}$

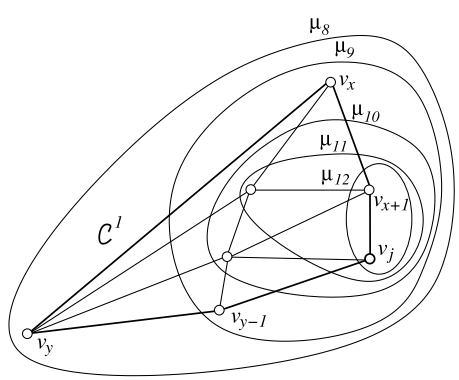

(a)

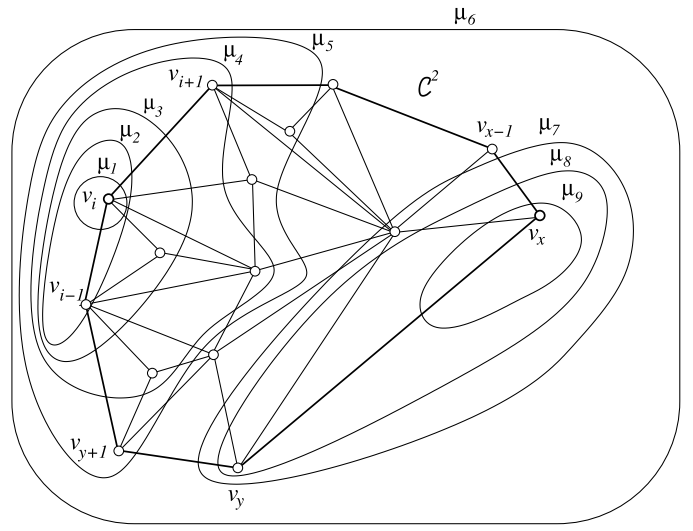

(b)

Fig. 5 The linearly-ordered outerclustered graphs obtained by splitting the linearly-ordered outerclustered graph of Fig. 3 by chord $\left(v_{x}, v_{y}\right)$. (a) $C^{1}\left(G^{1}, T^{1}\right)$. (b) $C^{2}\left(G^{2}, T^{2}\right)$ 
exactly twice. Namely, the boundary of every cluster $\mu$ is a simple closed curve, and hence it intersects $\mathcal{C}^{1}$ an even number of times. Suppose that the boundary of $\mu$ does not intersect $\mathcal{C}^{1}$. Then, since $\mu$ contains vertices of $G^{1}$, it contains all such vertices. Suppose that $\mu$ intersects $\mathcal{C}^{1}$ at least four times. At most two of such intersections can be on the edges of $\mathcal{C}^{1} \backslash\left(v_{x}, v_{y}\right)$, since $C$ is an outerclustered graph. Then, the boundary of $\mu$ intersects $\left(v_{x}, v_{y}\right)$ at least twice, contradicting the $c$-planarity of $C$.

Consider the subsequence $\Sigma_{1}$ of $\Sigma$ induced by the clusters in $T^{1}$. Obtain a sequence $\Sigma_{1}^{\prime}$ by removing from $\Sigma_{1}$ all the clusters that contain all the vertices of $G^{1}$ and that are different from $\sigma\left(v_{x}, v_{x+1}, \ldots, v_{y}\right)$, if any such a cluster exists. We claim that $C^{1}$ is linearly-ordered according to $\Sigma_{1}^{\prime}$.

We prove that $C^{1}$ satisfies Property LO1 of Definition 2. By definition, $\Sigma_{1}^{\prime}$ contains all the clusters of $T^{1}$ that contain vertices of $G^{1}$, except for each cluster $\mu$ that contains all the vertices of $G^{1}$ and that is different from $\sigma\left(v_{x}, v_{x+1}, \ldots, v_{y}\right)$. However, for any vertex $v$ of $G^{1}, \sigma(v) \neq \mu$, because $\sigma\left(v_{x}, v_{x+1}, \ldots, v_{y}\right)$ is a descendant of $\mu$ and contains $v$.

We prove that $C^{1}$ satisfies Property LO2. Since $C$ is linearly-ordered, $o(G)$ is delimited by two monotone paths $\mathcal{P}_{1}=\left(v_{i}, v_{i+1}, \ldots, v_{j}\right)$ and $\mathcal{P}_{2}=\left(v_{i}, v_{i-1}, \ldots, v_{j}\right)$. Suppose that (see Fig. 6(a)) both $v_{x}$ and $v_{y}$ belong to $\mathcal{P}_{1}$ and $v_{x}$ precedes $v_{y}$ in $\mathcal{P}_{1}$ (the other cases in which both $v_{x}$ and $v_{y}$ belong to $\mathcal{P}_{1}$ or both belong to $\mathcal{P}_{2}$ being analogous); then, the subpath of $\mathcal{P}_{1}$ between $v_{x}$ and $v_{y}$ and edge $\left(v_{x}, v_{y}\right)$ are monotone paths delimiting $o\left(G^{1}\right)$; further, $\mathcal{P}_{2}$ and the path obtained from $\mathcal{P}_{1}$ by replacing the subpath between $v_{x}$ and $v_{y}$ with edge $\left(v_{x}, v_{y}\right)$ are monotone paths delimiting $o\left(G^{2}\right)$. Suppose that $v_{x}$ belongs to $\mathcal{P}_{1}$ and $v_{y}$ belongs to $\mathcal{P}_{2}$. If $\sigma\left(v_{x}\right)$ precedes $\sigma\left(v_{y}\right)$ in $\Sigma$ (see Fig. 6(b)), then the two monotone paths delimiting $o\left(G^{1}\right)$ are (i) the subpath of $\mathcal{P}_{1}$ between $v_{x}$ and $v_{j}$ and (ii) edge $\left(v_{x}, v_{y}\right)$ plus the subpath of $\mathcal{P}_{2}$ between $v_{y}$ and $v_{j}$; the two monotone paths delimiting $o\left(G^{2}\right)$ are (i) the subpath of $\mathcal{P}_{1}$ between $v_{i}$ and $v_{x}$ plus edge $\left(v_{x}, v_{y}\right)$ and (ii) the subpath of $\mathcal{P}_{2}$ between $v_{i}$ and $v_{y}$. If $\sigma\left(v_{y}\right)$ precedes $\sigma\left(v_{x}\right)$ in $\Sigma$, then the two monotone paths delimiting $o\left(G^{1}\right)$ are (i) edge $\left(v_{y}, v_{x}\right)$ plus the subpath of $\mathcal{P}_{1}$ between $v_{x}$ and $v_{j}$ and (ii) the subpath of $\mathcal{P}_{2}$ between $v_{y}$ and $v_{j}$;

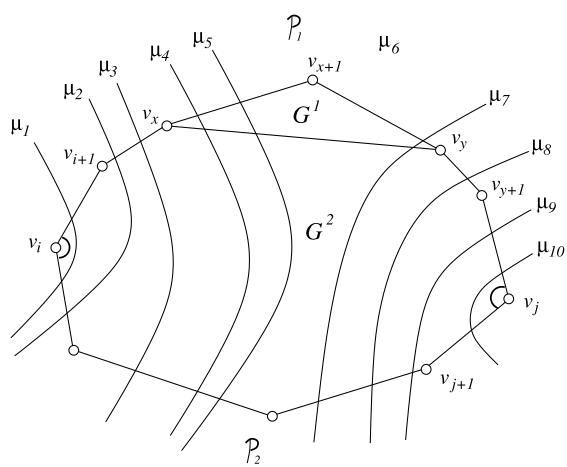

(a)

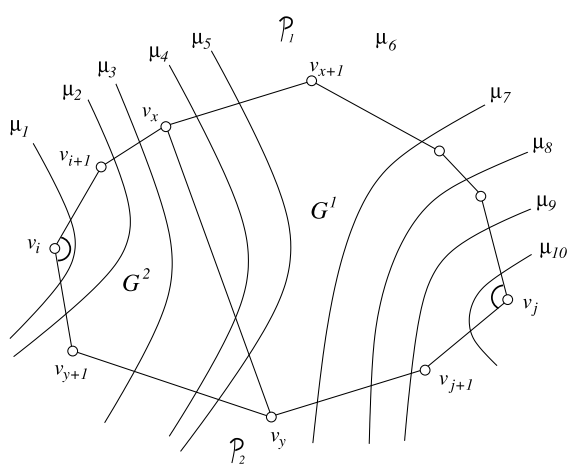

(b)

Fig. $6 C^{1}$ and $C^{2}$ satisfy LO2. (a) $v_{x}$ and $v_{y}$ belong to $\mathcal{P}_{1}$, and $v_{x}$ precedes $v_{y}$ in $\mathcal{P}_{1}$. (b) $v_{x}$ belongs to $\mathcal{P}_{1}, v_{y}$ belongs to $\mathcal{P}_{2}$, and $\sigma\left(v_{x}\right)$ precedes $\sigma\left(v_{y}\right)$ in $\Sigma$ 
the two monotone paths delimiting $o\left(G^{2}\right)$ are (i) the subpath of $\mathcal{P}_{1}$ between $v_{i}$ and $v_{x}$ and (ii) the subpath of $\mathcal{P}_{2}$ between $v_{i}$ and $v_{y}$ plus edge $\left(v_{y}, v_{x}\right)$.

We prove that $C^{1}$ satisfies Property LO3. Since $C$ is linearly-ordered according to $\Sigma$ and $\Sigma_{1}^{\prime}$ is a subsequence of $\Sigma$, it suffices to show that any two consecutive clusters $\mu_{x}$ and $\mu_{y}$ of $\Sigma_{1}^{\prime}$ are adjacent in $T^{1}$. Suppose, for a contradiction, that $\mu_{y}$ and $\mu_{x}$ are not adjacent in $T^{1}$. First, consider the case in which $\mu_{x}$ and $\mu_{y}$ are comparable, that is, $\mu_{y}$ is either an ancestor or a descendant of $\mu_{x}$. If $\mu_{y}$ is an ancestor of $\mu_{x}$ (the case in which $\mu_{x}$ is an ancestor of $\mu_{y}$ being analogous), consider the parent $\mu_{x+1}$ of $\mu_{x}$. Such a cluster contains all the vertices contained in $\mu_{x}$, hence either $\mu_{x+1}$ belongs to $\Sigma_{1}^{\prime}$, contradicting the fact that $\mu_{x}$ and $\mu_{y}$ are consecutive in $\Sigma_{1}^{\prime}$, or $\mu_{x+1}$ contains all the vertices of $G^{1}$ and is different from $\sigma\left(v_{x}, v_{x+1}, \ldots, v_{y}\right)$. However, this would imply that also $\mu_{y}$ contains all the vertices of $G^{1}$ and is different from $\sigma\left(v_{x}, v_{x+1}, \ldots, v_{y}\right)$, a contradiction to the fact that $\mu_{y}$ is in $\Sigma_{1}^{\prime}$. Second, consider the case in which $\mu_{x}$ and $\mu_{y}$ are incomparable, that is, $\mu_{y}$ is neither an ancestor nor a descendant of $\mu_{x}$. Again, consider the parent $\mu_{x+1}$ of $\mu_{x}$. Such a cluster contains all the vertices contained in $\mu_{x}$, still not being in $\Sigma_{1}^{\prime}$. Hence, $\mu_{x+1}$ contains all the vertices of $G^{1}$ and is different from $\sigma\left(v_{x}, v_{x+1}, \ldots, v_{y}\right)$; this implies that $\mu_{x}=\sigma\left(v_{x}, v_{x+1}, \ldots, v_{y}\right)$, hence $\mu_{y}$ is a descendant of $\mu_{x}$, a contradiction.

Let $C(G, T), C^{1}\left(G^{1}, T^{1}\right)$, and $C^{2}\left(G^{2}, T^{2}\right), \Sigma, v_{x}$, and $v_{y}$ be defined as above. Let $\Gamma$ be any convex-separated drawing of $C_{o}$ and let $P$ be the polygon representing $o(G)$ in $\Gamma$; let $v_{i}$ and $v_{j}$ be any two vertices with $\sigma\left(v_{i}\right)=\mu_{1}$ and $\sigma\left(v_{j}\right)=\mu_{k}$ such that the angle of $P$ incident to $v_{i}$ and the angle of $P$ incident to $v_{j}$ are strictly less than $180^{\circ}$ (such vertices exist by Property CS2 of Definition 3). Suppose that $v_{x}$ and $v_{y}$ are not collinear with any vertex of $o(G)$. Let $\Gamma_{1}$ and $\Gamma_{2}$ be the drawings of $C_{o}^{1}$ and $C_{o}^{2}$ obtained by drawing $\left(v_{x}, v_{y}\right)$ in $\Gamma$ as a straight-line segment. Denote by $P^{1}$ and $P^{2}$ the polygons representing $o\left(G^{1}\right)$ and $o\left(G^{2}\right)$ in $\Gamma_{1}$ and in $\Gamma_{2}$, respectively.

\section{Lemma $3 \Gamma_{1}$ and $\Gamma_{2}$ are convex-separated drawings.}

Proof We prove the statement for $\Gamma_{1}$, the proof for $\Gamma_{2}$ being analogous. Refer to Fig. 7. The drawing is straight-line and rectangular by construction. Since $\Gamma$ is a convex-separated drawing, by Property CS1 of Definition 3, the polygon $P$ representing $o(G)$ in $\Gamma$ is convex. Further, by hypothesis, $v_{x}$ and $v_{y}$ are not collinear with any vertex of $G$. Hence, $P^{1}$ is convex, thus satisfying Property CS1.

Drawing $\Gamma_{1}$ has no region-region crossings, since each cluster is represented in $\Gamma_{1}$ by the same rectangle as in $\Gamma$. We prove that $\Gamma_{1}$ has no edge-region crossings. Refer to Fig. 8. Suppose that an edge-region crossing exists between an edge $e$ and a cluster $v$. Then, $e$ is edge $\left(v_{x}, v_{y}\right)$, otherwise $\Gamma$ would not be $c$-planar. Cluster $v$ does not contain both of $v_{x}$ and $v_{y}$ otherwise, by the convexity of $v, e$ would be internal to $v$; further, $v$ does not contain exactly one of $v_{x}$ and $v_{y}$ otherwise, by the convexity of $v, e$ would cross $v$ exactly once. It follows that $v$ contains neither $v_{x}$ nor $v_{y}$. Consider the parent $\mu$ of $v$ in $T_{1}$. Such a parent exists, otherwise $v$ would be the root of $T$, contradicting the fact that $v$ contains neither $v_{x}$ nor $v_{y}$. Since $\Gamma$ satisfies Property CS3, there exists a convex region $R(\mu, v)$ with the properties described in 


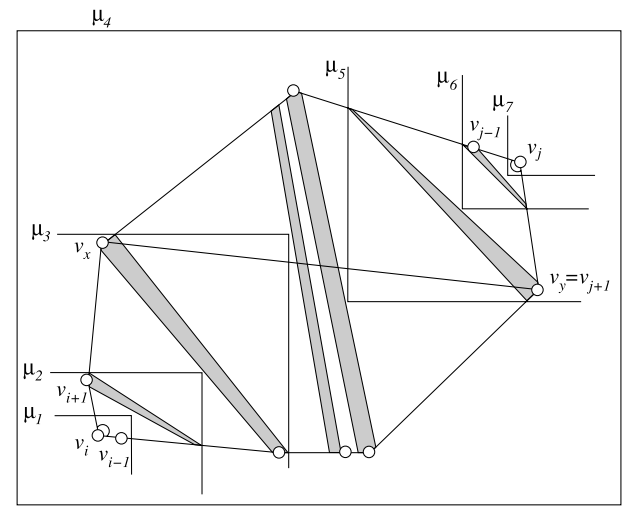

(a)

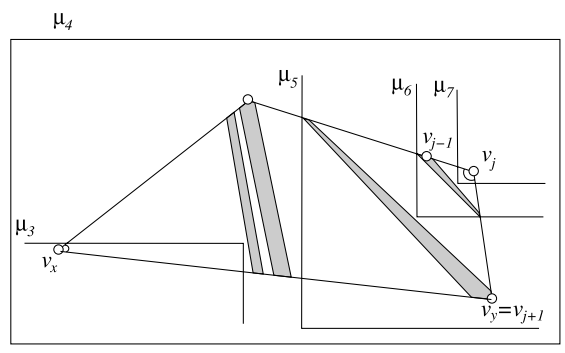

(b)

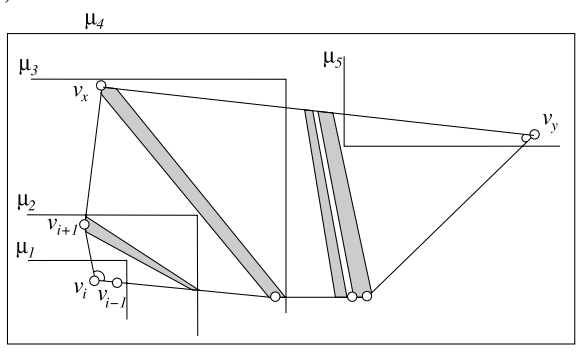

(c)

Fig. 7 (a) A convex-separated drawing $\Gamma$ of the outer face $C_{o}$ of a linearly-ordered outerclustered graph $C$. (b) and (c) Convex-separated drawings $\Gamma_{1}$ and $\Gamma_{2}$ of the outer faces $C_{o}^{1}$ and $C_{o}^{2}$ of the linearly-ordered outerclustered graphs $C^{1}$ and $C^{2}$

Fig. 8 Drawing $\Gamma_{1}$ has no crossing between an edge $e$ and a cluster containing none of the end-vertices of $e$. The thick line represents segment $\overline{p\left(l_{1}\right) p\left(l_{2}\right)}$

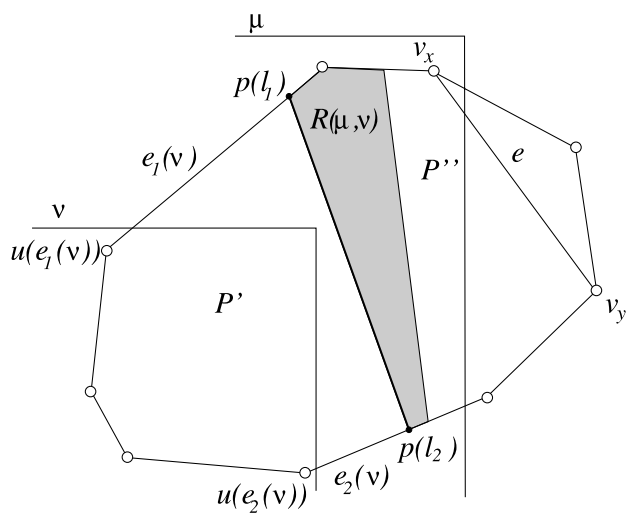

Definition 3 which "separates" $v$ from the rest of the drawing, thus avoiding an edgeregion crossing between $e$ and $v$. More precisely, since $\Gamma$ satisfies Property $\mathrm{O} 2$ of Definition $1, v$ has exactly two incident edges $e_{1}(v)$ and $e_{2}(v)$ belonging to $o(G)$. Denote by $u\left(e_{1}(v)\right)$ and $u\left(e_{2}(v)\right)$ the end-vertices of $e_{1}(v)$ and $e_{2}(v)$ belonging to $v$. Denote by $p\left(l_{1}\right)$ the endpoint of $l_{1}(\mu, v)$ that lies on $e_{1}(v)$ (if both endpoints of 
$l_{1}(\mu, v)$ lie on $e_{1}(v)$, then $p\left(l_{1}\right)$ is the one that is closer to $\left.u\left(e_{1}(v)\right)\right)$. Note that an endpoint of $l_{1}(\mu, v)$ lying on $e_{1}(\nu)$ exists as $\Gamma$ satisfies Property CS3. Analogously define $p\left(l_{2}\right)$. Then, segment $\overline{p\left(l_{1}\right) p\left(l_{2}\right)}$ splits $P$ into two disjoint convex polygons $P^{\prime}$ and $P^{\prime \prime}$, where $P^{\prime}$ contains all and only the vertices in $v$ and $P^{\prime \prime}$ contains all and only the vertices not in $v$, as $\Gamma$ satisfies Property CS3. By the convexity of $P^{\prime}$ and $P^{\prime \prime}, e$ is internal to $P^{\prime \prime}$, while the part of $v$ inside $P$ is internal to $P^{\prime}$. Hence, $e$ does not cross $v$.

We prove that $\Gamma_{1}$ satisfies Property CS2. First, observe that the angles incident to $v_{i}$ and $v_{j}$ in $P^{1}$ are strictly less than $180^{\circ}$, since they are strictly less than $180^{\circ}$ in $\Gamma$; further, the angles incident to $v_{x}$ and $v_{y}$ in $P^{1}$ are strictly less than $180^{\circ}$, since they are strictly less than the angles incident to $v_{x}$ and $v_{y}$ in $P$, that are at most $180^{\circ}$, by the convexity of $P$. Suppose that $\sigma\left(v_{x}\right)$ precedes $\sigma\left(v_{y}\right)$ in $\Sigma$, the opposite case being analogous. It suffices to observe that: (i) if $C^{1}$ contains both $v_{i}$ and $v_{j}$, then $\Sigma_{1}^{\prime}=\Sigma$; hence, $v_{i}$ and $v_{j}$ are vertices satisfying the desired properties; (ii) if $C^{1}$ contains neither $v_{i}$ nor $v_{j}$, then $\Sigma_{1}^{\prime}$ is the subsequence of $\Sigma$ that starts at $\sigma\left(v_{x}\right)$ and ends at $\sigma\left(v_{y}\right)$; hence, $v_{x}$ and $v_{y}$ are vertices satisfying the desired properties; (iii) if $C^{1}$ contains $v_{j}$ and does not contain $v_{i}$ (as in Fig. 7), then $\Sigma_{1}^{\prime}$ is the subsequence of $\Sigma$ that starts at $\sigma\left(v_{x}\right)$ and ends at $\sigma\left(v_{j}\right)=\mu_{k}$; hence, $v_{x}$ and $v_{j}$ are vertices satisfying the desired properties.

We prove that $\Gamma_{1}$ satisfies Property CS3. The existence of regions $R(\mu, v)$ inside $P^{1}$, for every cluster $v$, is easily deduced from the existence of regions $R(\mu, v)$ inside $P$, which is guaranteed as $\Gamma$ satisfies Property CS3. Namely, if $R(\mu, v)$ is not intersected by $e$, then a region $R(\mu, v)$ inside $P^{1}$ can be constructed coincident with the same region inside $P$. Further, if $R(\mu, v)$ is cut by $e$, then two regions are created, one inside $P^{1}$ and the other one inside $P^{2}$. The properties that have to be satisfied by $R(\mu, v)$ inside $P^{1}$ easily descend from the analogous properties satisfied by $R(\mu, v)$ inside $P$.

When dealing with outerclustered graphs and general clustered graphs, it is sufficient to consider clustered graphs whose underlying graphs have triangular outer faces. We exploit such a fact to refine the definition of convex-separated drawing of the outer face of a clustered graph into a similar definition that only deals with clustered graphs whose underlying graphs are 3-cycles (such a definition will be later used to ensure that a drawing of the outer face of a considered clustered graph satisfies certain properties). See Fig. 9.

Definition 4 Let $C(G, T)$ be a clustered graph such that $G$ is a 3-cycle $(u, v, z)$. A straight-line rectangular drawing $\Gamma(C)$ of $C$ is a triangular-convex-separated drawing if:

- TCS1: for every pair of clusters $\mu$ and $v$ such that $\mu$ is the parent of $v$ in $T$ and such that $\mu$ is not an ancestor of $\sigma(u, v, z)$, there exists a convex region $R(\mu, v)$ such that: (i) $R(\mu, v)$ is entirely contained inside $\mu \cap(P \cup \operatorname{int}(P))$, where $P$ is the triangle representing $G$ in $\Gamma(C)$; (ii) for any cluster $\mu^{\prime} \neq \mu$ and any child $\nu^{\prime}$ of $\mu^{\prime}, R(\mu, v)$ intersects neither $R\left(\mu^{\prime}, v^{\prime}\right)$ nor the boundary of $\mu^{\prime}$; (iii) $R(\mu, v) \cap P$ consists of two polygonal lines $l_{1}(\mu, \nu)$ and $l_{2}(\mu, v)$ such that at least one endpoint of $l_{1}(\mu, v)$ (resp. of $\left.l_{2}(\mu, v)\right)$ belongs to $e_{1}(v)$ (resp. to $\left.e_{2}(v)\right)$. 
Fig. 9 A triangularconvex-separated drawing of a clustered graph whose underlying graph is a 3-cycle

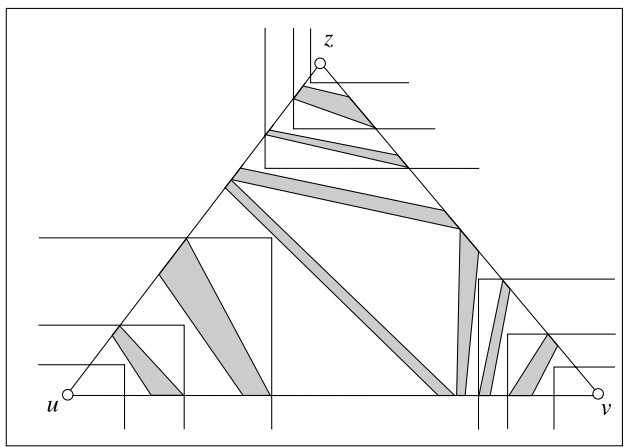

The interplay between a triangular-convex-separated drawing of a clustered graph whose underlying graph is a 3-cycle and a convex-separated drawing of a linearlyordered maximal outerclustered graph is clarified in the following lemma.

Lemma 4 Let $C(G, T)$ be a linearly-ordered maximal outerclustered graph. Then, a triangular-convex-separated drawing of $C_{o}$ is a convex-separated drawing of $C_{o}$.

Proof Consider any triangular-convex-separated drawing $\Gamma\left(C_{o}\right)$ of $C_{o}$. We prove that $\Gamma\left(C_{o}\right)$ is a convex-separated drawing. Properties CS1 and CS2 of Definition 3 easily descend from the fact that, since $o(G)$ is a 3-cycle, it is represented as a convex polygon $P$ whose internal angles are smaller than $180^{\circ}$. Property CS3 coincides with Property TCS1.

Finally, we define a class of drawings in which the properties of convexity and visibility among vertices and clusters are imposed on all the internal faces rather than on the outer face.

Definition 5 Let $C(G, T)$ be an internally-triangulated clustered graph. A drawing $\Gamma(C)$ of $C$ is an internally-convex-separated drawing if, for every internal face $f$ of $G$, the part $\Gamma\left(C_{f}\right)$ of $\Gamma(C)$ representing $C_{f}$ is a triangular-convex-separated drawing.

\section{Drawing Linearly-Ordered Outerclustered Graphs}

In this section we show how to construct an internally-convex-separated drawing of any linearly-ordered outerclustered graph $C$ for an arbitrary triangular-convexseparated drawing of the outer face $C_{o}$ of $C$. This is done by means of an inductive algorithm that uses the following lemma as the main tool (see Fig. 10):

Lemma 5 Let $C(G, T)$ be an internally-triangulated triconnected outerclustered graph. Suppose that $C$ is linearly-ordered according to a sequence $\mu_{1}, \mu_{2}, \ldots, \mu_{k}$ of clusters of T. Let $v_{i}$ and $v_{j}$ be any two vertices such that $\sigma\left(v_{i}\right)=\mu_{1}$ and $\sigma\left(v_{j}\right)=\mu_{k}$. Let $V_{1}\left(\right.$ resp. $\left.V_{2}\right)$ be the set of vertices between $v_{i}$ and $v_{j}$ (resp. between $v_{j}$ and $v_{i}$ ) in 
Fig. 10 A path satisfying Properties P1-P5

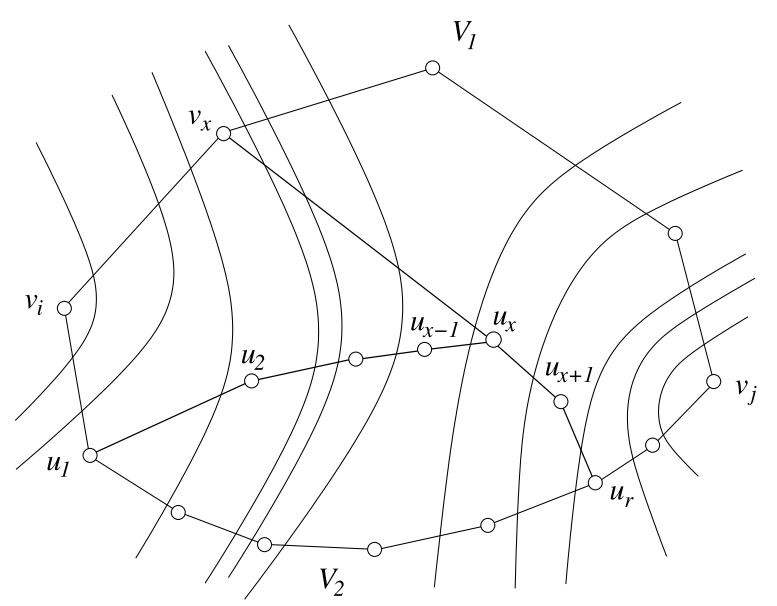

the clockwise order of the vertices around $o(G)$. Then, if $V_{1} \neq \emptyset$, there exists a path $\mathcal{P}_{u}=\left(u_{1}, u_{2}, \ldots, u_{r}\right)$ such that:

- P1: $u_{1}$ and $u_{r}$ belong to $V_{2} \cup\left\{v_{i}, v_{j}\right\}$;

- P2: $u_{i}$ is an internal vertex of $G$, for each $2 \leq i \leq r-1$;

- P3: if $\sigma\left(u_{i}\right)=\mu_{j_{1}}$ and $\sigma\left(u_{i+1}\right)=\mu_{j_{2}}$, then $j_{1}<j_{2}$, for each $1 \leq i \leq r-1$;

- P4: there exists exactly one vertex $u_{x}$, where $2 \leq x \leq r-1$, that is adjacent to at least one vertex $v_{x}$ in $V_{1}$;

- P5: there exists no chord among the vertices of path $\left(u_{1}, u_{2}, \ldots, u_{x}\right)$ and no chord among the vertices of path $\left(u_{x}, u_{x+1}, \ldots, u_{r}\right)$.

Proof We show an algorithm to construct a path $\mathcal{P}_{u}$ as required by the lemma. The algorithm derives path $\mathcal{P}_{u}$ in several steps. At step $s+1$ a path $\mathcal{P}^{s+1}$ is found by modifying the path $\mathcal{P}^{s}$ obtained at the previous step. At the last step $m$ of the algorithm, a path $\mathcal{P}_{u}$ can be obtained as a subpath of $\mathcal{P}^{m}$ satisfying the properties required by the lemma. A path satisfying Properties P1-P5 is shown in Fig. 10.

In more detail, at each step $s \leq m$, a path $\mathcal{P}^{s}=\left(u_{1}^{s}, u_{2}^{s}, \ldots, u_{r(s)}^{s}\right)$ is found satisfying the properties described below (see Fig. 11).

Denote by $\mathcal{P}_{1}=\left(v_{i}, v_{i+1}, \ldots, v_{j-1}, v_{j}\right)$ and $\mathcal{P}_{2}=\left(v_{i}, v_{i-1}, \ldots, v_{j+1}, v_{j}\right)$ the monotone paths on the vertices of $V_{1} \cup\left\{v_{i}, v_{j}\right\}$ and of $V_{2} \cup\left\{v_{i}, v_{j}\right\}$, respectively, delimiting $o(G)$.

- PP1: $u_{1}^{s}=v_{i}$ and $u_{r(s)}^{s}=v_{j}$;

- PP2: each vertex $u_{t}^{s}$ is either an internal vertex of $G$ or a vertex of $\mathcal{P}_{2}$, for each $2 \leq t \leq r(s)-1$;

- PP3: if $\sigma\left(u_{t}\right)=\mu_{j_{1}}$ and $\sigma\left(u_{t+1}\right)=\mu_{j_{2}}$, then $j_{1} \leq j_{2}$, for each $1 \leq t \leq r(s)-1$; and

- PP4: there exists no chord inside cycle $\mathcal{P}^{s} \cup \mathcal{P}_{1}$.

At the first step, set $\mathcal{P}^{1}=\mathcal{P}_{2}$. Path $\mathcal{P}^{1}$ satisfies Properties PP1 and PP2 by definition, Property PP3 since $C$ satisfies Property LO2 of Definition 2, and Property PP4 because $G$ is triconnected. 


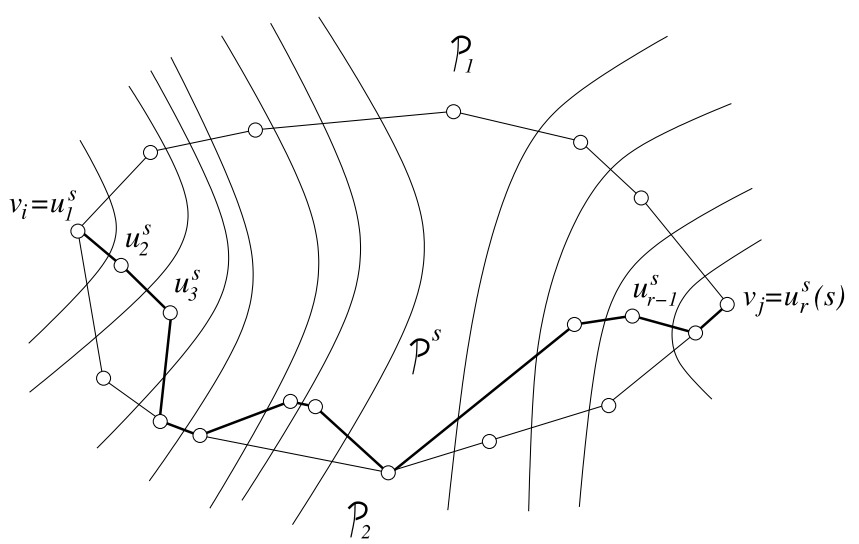

Fig. 11 A path $\mathcal{P}^{s}$ satisfying Properties PP1-PP4

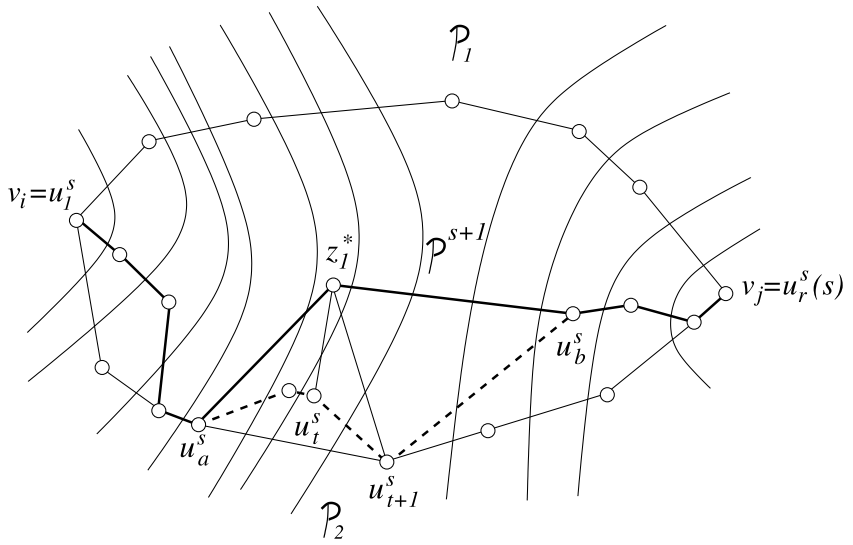

Fig. 12 Obtaining $\mathcal{P}^{s+1}$ from $\mathcal{P}^{s}$ if $\sigma\left(u_{a}^{s}\right), \sigma\left(z_{1}^{*}\right)$, and $\sigma\left(u_{b}^{s}\right)$ appear in this order in $\Sigma$. The dashed segments show the subpath of $\mathcal{P}^{s}$ that does not belong to $\mathcal{P}^{s+1}$

Suppose that a path $\mathcal{P}^{s}$ has been found at step $s$ of the algorithm. We show how to determine $\mathcal{P}^{s+1}$ at step $s+1$ of the algorithm.

Consider any edge $\left(u_{t}^{s}, u_{t+1}^{s}\right)$ of $\mathcal{P}^{s}$. Such an edge is incident to a face internal to cycle $\mathcal{P}^{s} \cup \mathcal{P}_{1}$. Let $z_{1}^{*}$ be the third vertex of such a face. As $\mathcal{P}^{s}$ satisfies Property PP4, $z_{1}^{*}$ is neither a vertex of $\mathcal{P}_{1}$ nor a vertex of $\mathcal{P}^{s}$. Hence, $z_{1}^{*}$ is internal to $\mathcal{P}^{s} \cup \mathcal{P}_{1}$.

Denote by $u_{a}^{s}$ and by $u_{b}^{s}$ the first and the last vertex of $\mathcal{P}^{s}$ adjacent to $z_{1}^{*}$. We distinguish two cases.

In the first case, $\sigma\left(u_{a}^{s}\right), \sigma\left(z_{1}^{*}\right)$, and $\sigma\left(u_{b}^{s}\right)$ appear in this order in $\Sigma$. Then, path $\mathcal{P}^{s+1}$ is obtained by replacing the subpath of $\mathcal{P}^{s}$ between $u_{a}^{s}$ and $u_{b}^{s}$ with path $\left(u_{a}^{s}, z_{1}^{*}, u_{b}^{s}\right)$. An example of this case is shown in Fig. 12 .

Suppose that $z_{1}^{*}$ is adjacent to at least one vertex in $V_{1}$. Since the first and the last vertex of $\mathcal{P}^{s+1}$ (that are $v_{i}$ and $v_{j}$, respectively) belong to $\mathcal{P}_{2}$, the vertices shared by $\mathcal{P}^{s+1}$ and $\mathcal{P}_{2}$ partition $\mathcal{P}^{s+1}$ into subpaths, where the internal vertices of each 


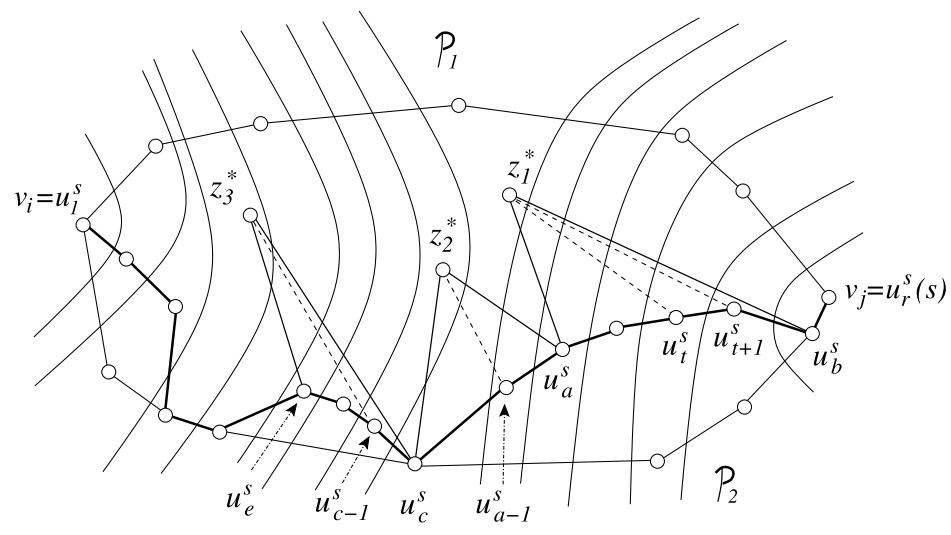

Fig. 13 Three repetitions of the argument for the case in which $\sigma\left(z_{1}^{*}\right)$ (resp. $\sigma\left(z_{2}^{*}\right)$ and $\sigma\left(z_{3}^{*}\right)$ ) precedes the smallest clusters containing the first and last neighbor of $z_{1}^{*}$ (resp. of $z_{2}^{*}$ or of $z_{3}^{*}$ ) in $\mathcal{P}^{s}$. Thick segments represent $\mathcal{P}^{s}$

subpath are internal to $\mathcal{P}_{1} \cup \mathcal{P}_{2}$. Let $\mathcal{P}_{u}$ be the one of such subpaths containing $z_{1}^{*}$. Path $\mathcal{P}_{u}=\left(u_{1}, u_{2}, \ldots, u_{x}, u_{x+1}, \ldots, u_{r}\right)$ defined as above is easily shown to satisfy Properties P1-P4 (where $u_{x}=z_{1}^{*}$ ). In particular, notice that $\mathcal{P}_{u}$ satisfies Property P3 since $\mathcal{P}^{s+1}$ satisfies Property PP3 and no two adjacent vertices of $\mathcal{P}_{u}$ have the same smallest containing cluster, as $C$ satisfies Property O3 of Definition 1. Further, if there exists a chord $\left(u_{a}, u_{b}\right)$ among the vertices of path $\left(u_{1}, u_{2}, \ldots, u_{x}\right)$ (resp. among the vertices of path $\left.\left(u_{x}, u_{x+1}, \ldots, u_{r}\right)\right)$, then replace the subpath of $\left(u_{1}, u_{2}, \ldots, u_{x}\right)$ (resp. of $\left.\left(u_{x}, u_{x+1}, \ldots, u_{r}\right)\right)$ between $u_{a}$ and $u_{b}$ with such a chord. The repetition of such an argument eventually leads to a path $\mathcal{P}_{u}$ also satisfying Property P5. Hence, the algorithm stops because a path with the properties required by the lemma has been found.

Suppose that $z_{1}^{*}$ is not adjacent to any vertex in $V_{1}$. Then, $\mathcal{P}^{s+1}$ is easily shown to satisfy Properties PP1-PP4. Hence, the algorithm continues with step $s+2$.

In the second case, either $\sigma\left(z_{1}^{*}\right), \sigma\left(u_{a}^{s}\right)$, and $\sigma\left(u_{b}^{s}\right)$ appear in this order in $\Sigma$, or they appear in the order $\sigma\left(u_{a}^{s}\right), \sigma\left(u_{b}^{s}\right), \sigma\left(z_{1}^{*}\right)$. Suppose that $\sigma\left(z_{1}^{*}\right), \sigma\left(u_{a}^{s}\right)$, and $\sigma\left(u_{b}^{s}\right)$ appear in this order in $\Sigma$, the other case being analogous. See Fig. 13.

Consider edge $\left(u_{a-1}^{s}, u_{a}^{s}\right)$ of $\mathcal{P}^{s}$. Such an edge is incident to a face internal to cycle $\mathcal{P}^{s} \cup \mathcal{P}_{1}$. Let $z_{2}^{*}$ be the third vertex of such a face. By Property PP4, $z_{2}^{*}$ is neither a vertex of $\mathcal{P}_{1}$ nor a vertex of $\mathcal{P}^{s}$. Hence, $z_{2}^{*}$ is internal to $\mathcal{P}^{s} \cup \mathcal{P}_{1}$. Further, $\sigma\left(z_{2}^{*}\right)$ is not the same cluster of $\sigma\left(u_{a}^{s}\right)$ (since $C$ satisfies Property O3 of Definition 1), and $\sigma\left(z_{2}^{*}\right.$ ) does not follow $\sigma\left(u_{a}^{s}\right)$ in $\Sigma$, otherwise edge $\left(u_{a}^{s}, z_{2}^{*}\right)$ would cross twice the boundary of $\sigma\left(u_{a}^{s}\right)$ or the boundary of the cluster coming before $\sigma\left(u_{a}^{s}\right)$ in $\Sigma$ (depending on whether $\sigma\left(u_{a}^{s}\right)$ is a child or is the parent of the cluster coming before $\sigma\left(u_{a}^{s}\right)$ in $\Sigma$ ). Hence, $\sigma\left(z_{2}^{*}\right)$ precedes $\sigma\left(u_{a}^{s}\right)$ in $\Sigma$.

Then, the whole argument can be repeated, namely denote by $u_{c}^{s}$ and by $u_{d}^{s}$ the first and the last vertex adjacent to $z_{2}^{*}$ in $\mathcal{P}^{s}$. Since $C$ satisfies Property O3, then $\sigma\left(u_{c}^{s}\right) \neq \sigma\left(z_{2}^{*}\right)$; since $\sigma\left(z_{2}^{*}\right)$ precedes $\sigma\left(u_{a}^{s}\right)$ in $\Sigma$ and since $\sigma\left(u_{a}^{s}\right)$ does not follow $\sigma\left(u_{d}^{s}\right)$ in $\Sigma$, then $\sigma\left(z_{2}^{*}\right)$ precedes $\sigma\left(u_{d}^{s}\right)$ in $\Sigma$. Then, either $\sigma\left(u_{c}^{s}\right)$ precedes $\sigma\left(z_{2}^{*}\right)$ in $\Sigma$ or $\sigma\left(z_{2}^{*}\right)$ precedes $\sigma\left(u_{c}^{s}\right)$ in $\Sigma$. If $\sigma\left(u_{c}^{s}\right)$ precedes $\sigma\left(z_{2}^{*}\right)$, then a path $\mathcal{P}^{s+1}$ is found 
by replacing the subpath of $\mathcal{P}^{s}$ between $u_{c}^{s}$ and $u_{d}^{s}$ with path $\left(u_{c}^{s}, z_{2}^{*}, u_{d}^{s}\right)$; then, either a path $\mathcal{P}_{u}$ satisfying Properties P1-P5 can be derived from $\mathcal{P}^{s+1}$ or $\mathcal{P}^{s+1}$ satisfies Properties PP1-PP4, depending on whether $z_{2}^{*}$ is adjacent to at least one vertex in $V_{1}$ or not. If $\sigma\left(z_{2}^{*}\right)$ precedes $\sigma\left(u_{c}^{s}\right)$, then edge $\left(u_{c-1}^{s}, u_{c}^{s}\right)$ and the vertex $z_{3}^{*}$ incident to the face $\left(u_{c-1}^{s}, u_{c}^{s}, z_{3}^{*}\right)$ internal to cycle $\mathcal{P}^{s} \cup \mathcal{P}_{1}$ are considered and the argument is repeated again. The repetition of such an argument eventually leads to find a vertex $z_{f}^{*}$ that is incident to a face $\left(u_{y-1}^{s}, u_{y}^{s}, z_{f}^{*}\right)$ and such that, denoting by $u_{p}^{s}$ and $u_{q}^{s}$ the first and the last neighbor of $z_{f}^{*}$ in $\mathcal{P}^{s}, \sigma\left(u_{p}^{s}\right), \sigma\left(z_{f}^{*}\right)$, and $\sigma\left(u_{q}^{s}\right)$ appear in this order in $\Sigma$. Namely, at every repetition of such an argument, the considered edge (that is equal to $\left(u_{t}^{s}, u_{t+1}^{s}\right)$ at the first repetition, to $\left(u_{a-1}^{s}, u_{a}^{s}\right)$ at the second repetition, to $\left(u_{c-1}^{s}, u_{c}^{s}\right)$ at the third repetition, and to $\left(u_{y-1}^{s}, u_{y}^{s}\right)$ at the last repetition) gets closer to $v_{i}$ in $\mathcal{P}^{s}$; then, after a certain number of repetitions of the algorithm, vertex $u_{y-1}^{s}$ eventually belongs to cluster $\mu_{1}$ and no cluster preceding $\mu_{1}$ exists in $\Sigma$.

It remains to observe that, after a certain number $m$ of steps of the algorithm, a path $\mathcal{P}_{u}$ satisfying Properties P1-P5 can be derived from $\mathcal{P}^{m}$. Namely, the number of vertices internal to cycle $\mathcal{P}^{s} \cup \mathcal{P}_{1}$ decreases at every step of the algorithm, hence a vertex $z^{*}$ adjacent to a vertex in $V_{1}$ is eventually added to a path $\mathcal{P}^{m-1}$ to form a path $\mathcal{P}^{m}$, from which a path $\mathcal{P}_{u}$ satisfying Properties P1-P5 can then be derived. Notice that $z^{*}$ is the only vertex of $\mathcal{P}^{m}$ adjacent to a vertex in $V_{1}$, since no vertex of $\mathcal{P}^{m-1}$ is adjacent to a vertex in $V_{1}$, as $\mathcal{P}^{m-1}$ satisfies Property PP4.

A pseudo-code description of the algorithm for finding a path $\mathcal{P}_{u}$ satisfying the properties required by Lemma 5 (supposing that $V_{1} \neq \emptyset$ ) is presented in Algorithm 1 .

A lemma similar to Lemma 5 is presented in the following. The proof of such a lemma can be obtained analogously to the one of Lemma 5 , where $V_{1}$ replaces $V_{2}$ and vice versa.

Lemma 6 Let $C(G, T)$ be an internally-triangulated triconnected outerclustered graph. Suppose that $C$ is linearly-ordered according to a sequence $\mu_{1}, \mu_{2}, \ldots, \mu_{k}$ of clusters of T. Let $v_{i}$ and $v_{j}$ be any two vertices such that $\sigma\left(v_{i}\right)=\mu_{1}$ and $\sigma\left(v_{j}\right)=\mu_{k}$. Let $V_{1}\left(\right.$ resp. $\left.V_{2}\right)$ be the set of vertices between $v_{i}$ and $v_{j}$ (resp. between $v_{j}$ and $v_{i}$ ) in the clockwise order of the vertices around $o(G)$. Then, if $V_{2} \neq \emptyset$, there exists a path $\mathcal{P}_{u}=\left(u_{1}, u_{2}, \ldots, u_{r}\right)$ such that:

- P1: $u_{1}$ and $u_{r}$ belong to $V_{1} \cup\left\{v_{i}, v_{j}\right\}$;

- P2: $u_{i}$ is an internal vertex of $G$, for each $2 \leq i \leq r-1$;

- P3: if $\sigma\left(u_{i}\right)=\mu_{j_{1}}$ and $\sigma\left(u_{i+1}\right)=\mu_{j_{2}}$, then $j_{1}<j_{2}$, for each $1 \leq i \leq r-1$;

- P4: there exists exactly one vertex $u_{x}$, where $2 \leq x \leq r-1$, that is adjacent to at least one vertex $v_{x}$ in $V_{2}$;

- P5: there exists no chord among the vertices of path $\left(u_{1}, u_{2}, \ldots, u_{x}\right)$ and no chord among the vertices of path $\left(u_{x}, u_{x+1}, \ldots, u_{r}\right)$.

When dealing with a linearly-ordered outerclustered graph $C$, we will always denote by $v_{i}$ and $v_{j}$ two vertices such that $\sigma\left(v_{i}\right)=\mu_{1}$ and $\sigma\left(v_{j}\right)=\mu_{k}$, where $\mu_{1}, \mu_{2}, \ldots, \mu_{k}$ is the sequence of clusters $C$ is linearly-ordered according to.

We now present the main theorem of this section. 


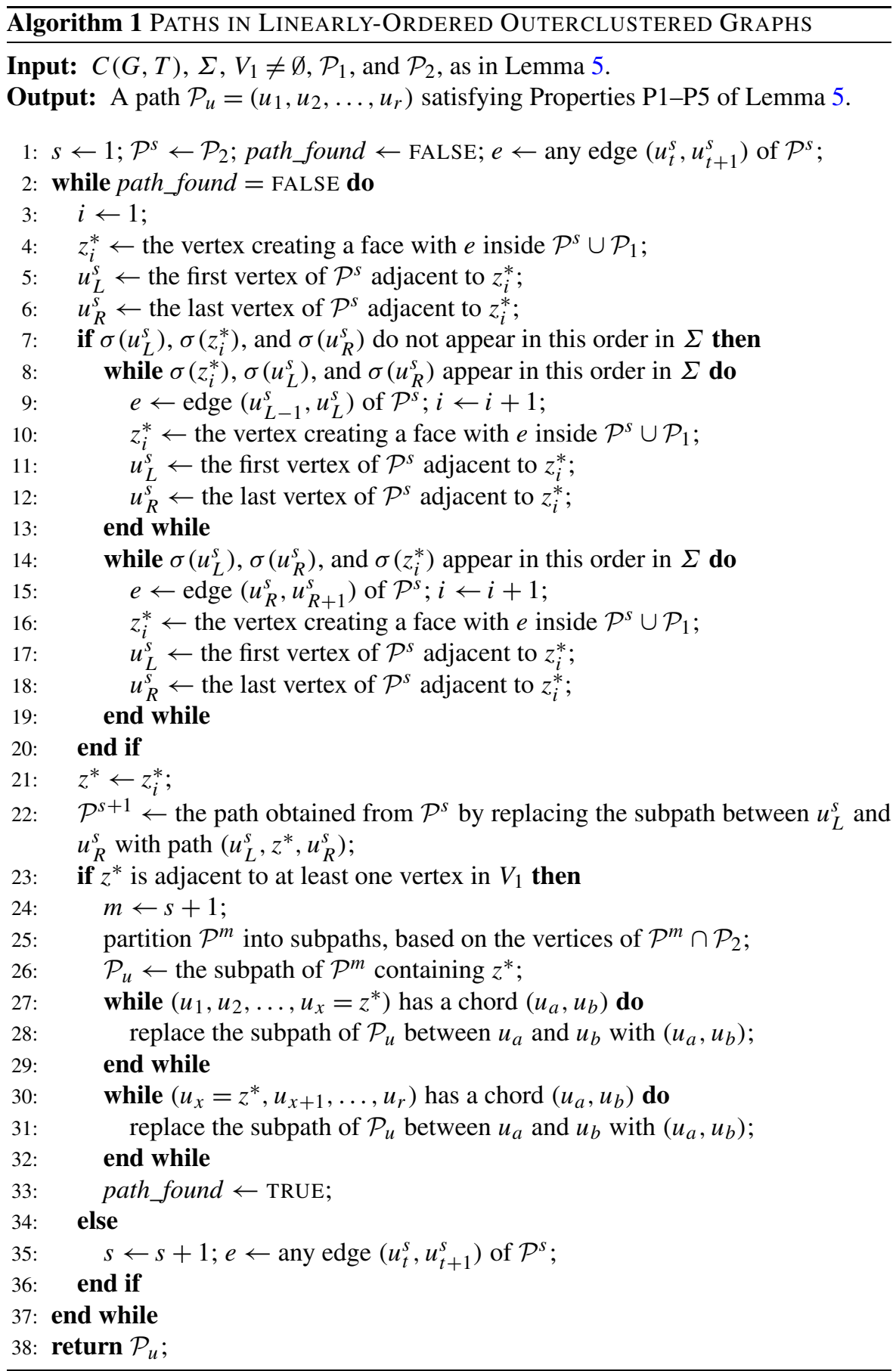


Theorem 1 Let $C(G, T)$ be a linearly-ordered internally-triangulated triconnected outerclustered graph. Then, for every convex-separated drawing $\Gamma\left(C_{o}\right)$ of $C_{o}$, there exists an internally-convex-separated drawing $\Gamma(C)$ of $C$ completing $\Gamma\left(C_{o}\right)$.

Proof We prove the statement by induction on the number of internal vertices of $G$. First observe that, since $G$ is triconnected, $o(G)$ has no chords. Hence, if $G$ has no internal vertices, then $C_{o}$ and $C$ are the same graph and the statement trivially follows. Otherwise, $G$ has internal vertices. Denote by $\mathcal{C}$ the cycle delimiting $o(G)$. We show how to split $C$ into smaller linearly-ordered internally-triangulated triconnected outerclustered graphs.

Let $\Gamma\left(C_{o}\right)$ be an arbitrary convex-separated drawing of $C_{o}$. Denote by $P$ the polygon representing $o(G)$ in $\Gamma\left(C_{o}\right)$. Since $\Gamma\left(C_{o}\right)$ satisfies Property CS1 of Definition 3, $P$ is convex. Suppose that $C$ is linearly-ordered according to a sequence $\Sigma=\mu_{1}, \mu_{2}, \ldots, \mu_{h}, \ldots, \mu_{k}$ of clusters of $T$. Let $v_{i}$ and $v_{j}$ be two vertices of $\mathcal{C}$ with $\sigma\left(v_{i}\right)=\mu_{1}$ and $\sigma\left(v_{j}\right)=\mu_{k}$ such that the angle of $P$ incident to $v_{i}$ and the angle of $P$ incident to $v_{j}$ are strictly less than $180^{\circ}$. Such vertices exist since $\Gamma\left(C_{o}\right)$ satisfies Property CS2 of Definition 3.

We distinguish two cases:

Case 1 applies when the vertices of $V_{1} \cup\left\{v_{i}, v_{j}\right\}$ are not all collinear. In such a case, $V_{1} \neq \emptyset$, otherwise the only two vertices of $V_{1} \cup\left\{v_{i}, v_{j}\right\}$ would be collinear. Hence, a path $\left(u_{1}, u_{2}, \ldots, u_{x}, \ldots, u_{r}\right)$ can be found as in Lemma 5 . Let $v_{x}$ be any vertex of $V_{1}$ adjacent to $u_{x}$.

Lemma 7 Vertex $v_{x}$ does not lie on the line $l\left(u_{1}, u_{r}\right)$ through $u_{1}$ and $u_{r}$.

Proof Denote by $\mathcal{P}\left(u_{1}, v_{x}, u_{r}\right)$ the subpath of $\mathcal{C}$ between vertices $u_{1}$ and $u_{r}$ containing $v_{x}$. Such a path is hence part of the boundary of $o(G)$. If there exists an internal vertex of $\mathcal{P}\left(u_{1}, v_{x}, u_{r}\right)$ lying on the segment $\overline{u_{1} u_{r}}$, then all the vertices in $\mathcal{P}\left(u_{1}, v_{x}, u_{r}\right)$ lie on $\overline{u_{1} u_{r}}$, otherwise polygon $P$ would not be convex (see Fig. 14(a)). However, $\mathcal{P}\left(u_{1}, v_{x}, u_{r}\right)$ contains all the vertices of $V_{1} \cup\left\{v_{i}, v_{j}\right\}$, that are not all collinear, by hypothesis. Now suppose that $v_{x}$ lies on the half-line that is part of $l\left(u_{1}, u_{r}\right)$, that starts at $u_{1}$, and that does not contain $u_{r}$ (the case in which $v_{x}$ lies on the half-line that is part of $l\left(u_{1}, u_{r}\right)$, that starts at $u_{r}$, and that does not contain $u_{1}$ being analogous). By the convexity of $P$, all the vertices in the path $\mathcal{P}\left(v_{x}, u_{1}, u_{r}\right)$ between $v_{x}$ and $u_{r}$ containing $u_{1}$ lie on $l\left(u_{1}, u_{r}\right)$ (see Fig. 14(b)). However, since

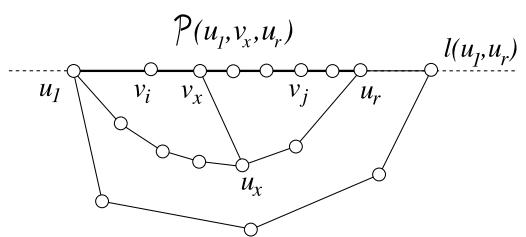

(a)

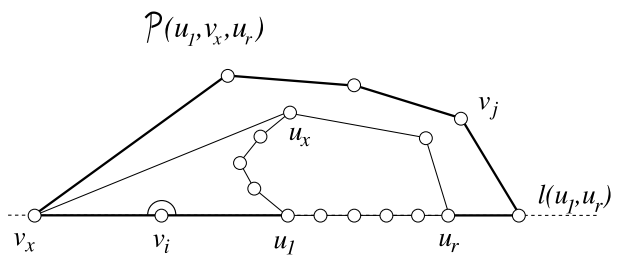

(b)

Fig. 14 Illustration for the proof of Lemma 7. (a) Vertex $v_{x}$ lies on $\overline{u_{1} u_{r}}$. (b) Vertex $v_{x}$ lies on the half-line that is part of $l\left(u_{1}, u_{r}\right)$, that starts at $u_{1}$, and that does not contain $u_{r}$ 
$v_{x} \in V_{1}$ and since $u_{1}, u_{r} \in V_{2} \cup\left\{v_{i}, v_{j}\right\}$, it follows that $v_{i}$ is one of the internal vertices of $\mathcal{P}\left(v_{x}, u_{1}, u_{r}\right)$, thus its incident angle in $P$ is exactly $180^{\circ}$, contradicting the hypothesis.

Consider the triangle $T\left(v_{x}, u_{1}, u_{r}\right)$ with vertices $v_{x}, u_{1}$, and $u_{r}$. By Lemma 7 , such a triangle is non-degenerate and, by the convexity of $P$, it is entirely contained inside $P$ (observe that parts of triangle $T\left(v_{x}, u_{1}, u_{r}\right)$ and of polygon $P$ could coincide). Denote by $\sigma^{\prime}\left(u_{i}\right)$ any cluster in $\Sigma$ which is a child of $\sigma\left(u_{i}\right)$, for each $2 \leq i \leq r-1$.

Lemma 8 There exists a small disk $D$ entirely contained inside $\operatorname{int}\left(T\left(v_{x}, u_{1}, u_{r}\right)\right) \cap$ $R\left(\sigma\left(u_{x}\right), \sigma^{\prime}\left(u_{x}\right)\right)$.

Proof Let $\mu_{j_{1}}, \mu_{j_{2}}, \mu_{j_{3}}$, and $\mu_{j_{4}}$ be clusters $\sigma\left(u_{1}\right), \sigma\left(u_{x}\right), \sigma\left(u_{r}\right)$, and $\sigma\left(v_{x}\right)$ in $\Sigma$, respectively. Denote by $\mu_{j}^{\prime}$ any cluster in $\Sigma$ which is a child of $\mu_{j}$. Since $2 \leq x \leq$ $r-1$ and since $\sigma\left(u_{i}\right)=\mu_{l_{1}}$ and $\sigma\left(u_{i+1}\right)=\mu_{l_{2}}$ implies $l_{1}<l_{2}$, we have $j_{1}<j_{2}<j_{3}$. Since $C$ satisfies Property O3 of Definition $1, \sigma\left(u_{x}\right) \neq \sigma\left(v_{x}\right)$ and hence $j_{2} \neq j_{4}$. Suppose that $j_{2}<j_{4}$, the case $j_{2}>j_{4}$ being analogous.

We claim that $s_{1}=R\left(\mu_{j_{2}}, \mu_{j_{2}}^{\prime}\right) \cap \overline{u_{1} u_{r}}$ and $s_{2}=R\left(\mu_{j_{2}}, \mu_{j_{2}}^{\prime}\right) \cap \overline{u_{1} v_{x}}$ are straightline segments. The claim implies the lemma, namely if $s_{1}$ and $s_{2}$ are straight-line segments, then the interior of the quadrilateral having $s_{1}$ and $s_{2}$ as opposite sides entirely belongs to $\operatorname{int}\left(T\left(v_{x}, u_{1}, u_{r}\right)\right) \cap R\left(\mu_{j_{2}}, \mu_{j_{2}}^{\prime}\right)$ and any disk entirely contained inside such a quadrilateral satisfies the requirements of the lemma. See Fig. 15.

By Property CS3 of Definition $3, R\left(\mu_{j_{2}}, \mu_{j_{2}}^{\prime}\right) \cap P$ consists of two line segments $l_{1}\left(\mu_{j_{2}}, \mu_{j_{2}}^{\prime}\right)$ and $l_{2}\left(\mu_{j_{2}}, \mu_{j_{2}}^{\prime}\right)$. Since $j_{2} \neq j_{1}, j_{3}, j_{4}$, none of vertices $u_{1}, u_{r}$, and $v_{x}$

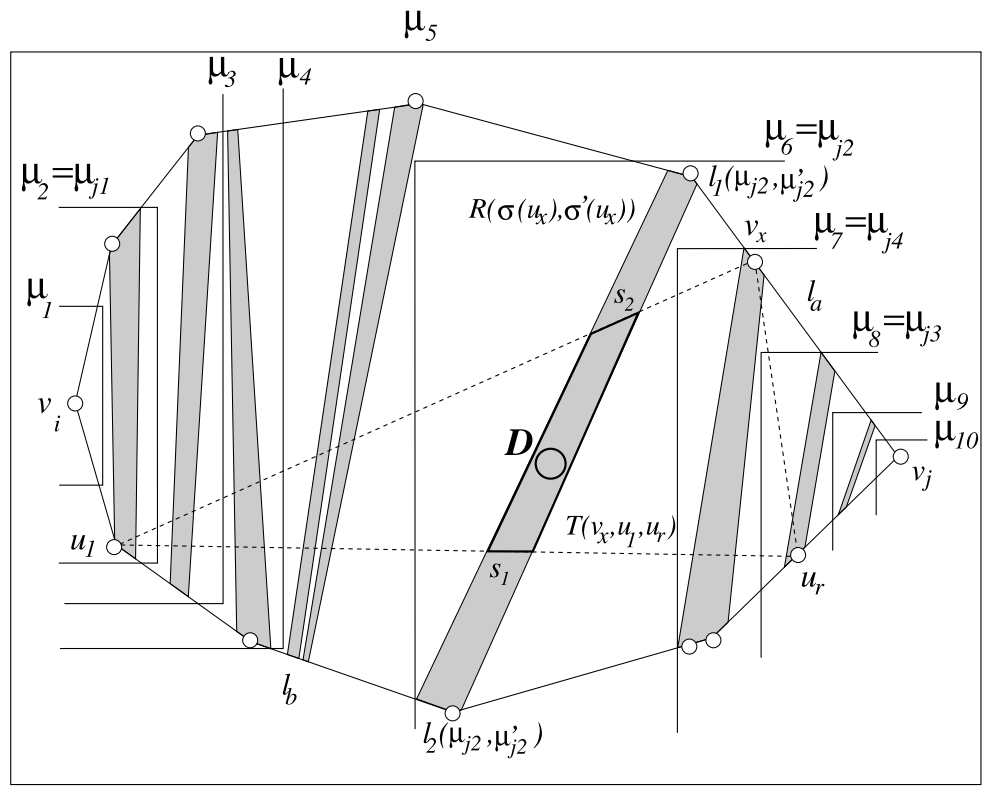

Fig. 15 Illustration for the proof of Lemma 8 
belongs to $R\left(\mu_{j_{2}}, \mu_{j_{2}}^{\prime}\right)$. Consider the two polygonal lines $l_{a}$ and $l_{b}$ obtained by removing $l_{1}\left(\mu_{j_{2}}, \mu_{j_{2}}^{\prime}\right)$ and $l_{2}\left(\mu_{j_{2}}, \mu_{j_{2}}^{\prime}\right)$ from $P$. Since $P$ is convex, it suffices to show that $u_{1}$ is in $l_{a}$ and $u_{r}$ is in $l_{b}$, or vice versa, in order to prove that $s_{1}$ is a straight-line segment; analogously, it suffices to show that $u_{1}$ is in $l_{a}$ and $v_{x}$ is in $l_{b}$, or vice versa, in order to prove that $s_{2}$ is a straight-line segment. However, this directly follows from the fact that one of the sides of region $R\left(\mu_{j_{2}}, \mu_{j_{2}}^{\prime}\right)$ separates the vertices whose smallest containing cluster is $\mu_{j}$, with $j<j_{2}$, from the vertices whose smallest containing cluster is $\mu_{j}$, with $j>j_{2}$.

Place $u_{x}$ at any point inside $D$. Draw edge $\left(u_{x}, v_{x}\right)$ as a straight-line segment. Consider the straight-line segment $\overline{u_{1} u_{x}}$ and consider any cluster $\mu_{j}$ such that $j_{1}<$ $j<j_{2}$. Then, $R\left(\mu_{j}, \mu_{j}^{\prime}\right) \cap \overline{u_{1} u_{x}}$ is a straight-line segment, namely one of the sides of region $R\left(\mu_{j}, \mu_{j}^{\prime}\right)$ separates the vertices whose smallest containing cluster is $\mu_{j^{*}}$, with $j^{*}<j$, from the vertices whose smallest containing cluster is $\mu_{j^{*}}$, with $j^{*}>j$. Analogously, consider the straight-line segment $\overline{u_{x} u_{r}}$ and consider any cluster $\mu_{j}$ such that $j_{2}<j<j_{3}$. Then, $R\left(\mu_{j}, \mu_{j}^{\prime}\right) \cap \overline{u_{x} u_{r}}$ is a straight-line segment, namely one of the sides of region $R\left(\mu_{j}, \mu_{j}^{\prime}\right)$ separates the vertices whose smallest containing cluster is $\mu_{j^{*}}$, with $j^{*}<j$, from the vertices whose smallest containing cluster is $\mu_{j^{*}}$, with $j^{*}>j$. Draw each vertex $u_{i}$, with $2 \leq i \leq x-1$, at any internal point of $\overline{u_{1} u_{x}} \cap R\left(\sigma\left(u_{i}\right), \sigma^{\prime}\left(u_{i}\right)\right)$. Analogously, draw each vertex $u_{i}$, with $x+1 \leq i \leq r-1$, at any point of $\overline{u_{x} u_{r}} \cap R\left(\sigma\left(u_{i}\right), \sigma^{\prime}\left(u_{i}\right)\right)$. Denote by $\Gamma$ the constructed drawing. The construction of $\Gamma$ is depicted in Fig. 16.

Let $\mathcal{C}_{1}$ be the cycle composed of path $\left(u_{1}, u_{2}, \ldots, u_{x}\right)$, of edge $\left(u_{x}, v_{x}\right)$, and of the path between $u_{1}$ and $v_{x}$ in $\mathcal{C}$ not containing $u_{r}$. Let $\mathcal{C}_{2}$ be the cycle composed of path

$\mu_{5}$

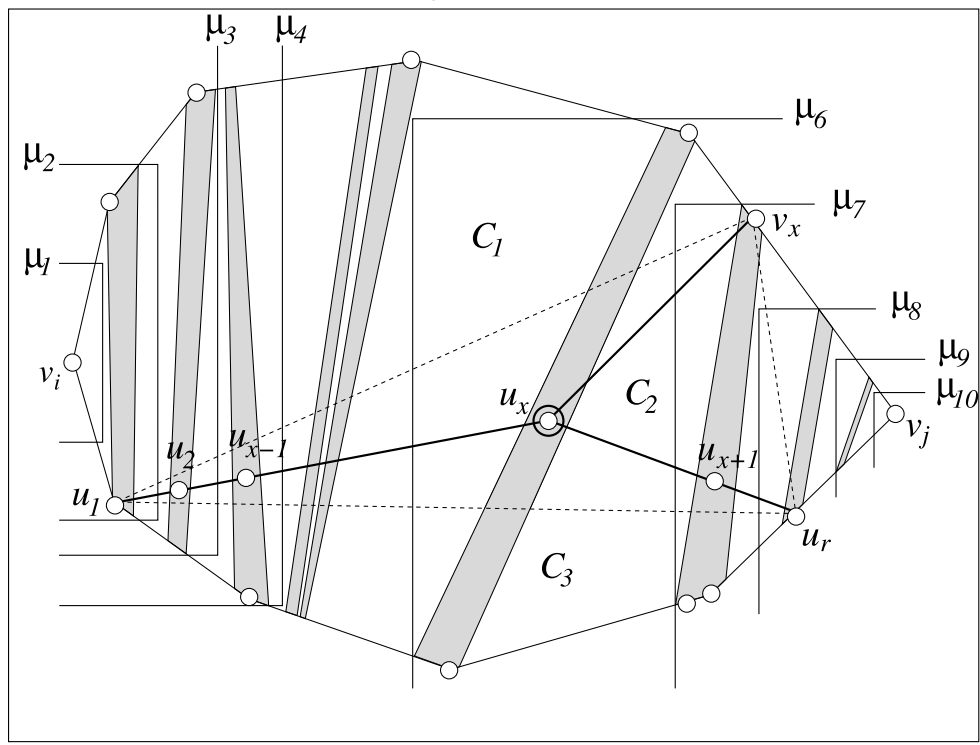

Fig. 16 Drawing path $\left(u_{1}, u_{2}, \ldots, u_{x}, \ldots, u_{r}\right)$ and edge $\left(u_{x}, v_{x}\right)$ 
$\left(u_{x}, u_{x+1}, \ldots, u_{r}\right)$, of edge $\left(u_{x}, v_{x}\right)$, and of the path between $v_{x}$ and $u_{r}$ in $\mathcal{C}$ not containing $u_{1}$. Let $\mathcal{C}_{3}$ be the cycle composed of path $\left(u_{1}, u_{2}, \ldots, u_{x}, \ldots, u_{r}\right)$ and of the path between $u_{1}$ and $u_{r}$ in $\mathcal{C}$ not containing $v_{x}$. Let $\mathcal{T}_{1}, \mathcal{T}_{2}$, and $\mathcal{T}_{3}$ be the subtrees of $T$ induced by the clusters containing vertices of $\mathcal{C}_{1}, \mathcal{C}_{2}$, and $\mathcal{C}_{3}$, respectively. Denote by $G_{1}, G_{2}$, and $G_{3}$ the subgraphs of $G$ induced by the vertices inside or on the border of $\mathcal{C}_{1}, \mathcal{C}_{2}$, and $\mathcal{C}_{3}$, respectively. Finally, let $C_{1}, C_{2}$, and $C_{3}$ be the clustered graphs whose underlying graphs are $G_{1}, G_{2}$, and $G_{3}$, respectively, and whose inclusion trees $T_{1}$, $T_{2}$, and $T_{3}$ are the subtrees of $T$ induced by the clusters containing vertices of $G_{1}$, $G_{2}$, and $G_{3}$, respectively.

Lemma $9 C_{1}, C_{2}$, and $C_{3}$ are linearly-ordered outerclustered graphs.

Proof Denote by $\mathcal{C}_{1,2}$ the cycle composed of path $\left(u_{1}, u_{2}, \ldots, u_{x}, \ldots, u_{r}\right)$ and of the path between $u_{1}$ and $u_{r}$ in $\mathcal{C}$ containing $v_{x}$. Let $C_{1,2}$ be the clustered graph whose underlying graph $G_{1,2}$ is the subgraph of $G$ whose vertices are incident to or internal to $\mathcal{C}_{1,2}$, and whose inclusion tree $T_{1,2}$ is the subtree of $T$ induced by the clusters containing vertices of $G_{1,2}$. It suffices to prove that $C_{1,2}$ and $C_{3}$ are linearly-ordered outerclustered graphs. Namely, by Lemma 2, if $C_{1,2}$ is a linearly-ordered outerclustered graph, then $C_{1}$ and $C_{2}$ are linearly-ordered outerclustered graphs, as well.

Since $C$ is linearly-ordered, $o(G)$ is delimited by two monotone paths $\mathcal{P}_{1}$ and $\mathcal{P}_{2}$, where $\mathcal{P}_{1}$ (resp. $\mathcal{P}_{2}$ ) has the vertices in $V_{1}$ (resp. in $V_{2}$ ) as internal vertices.

Consider the sequence $\Sigma=\mu_{1}, \mu_{2}, \ldots, \mu_{k}$ according to which $C$ is linearlyordered. Recall that clusters $\mu_{i}$ and $\mu_{i+1}$ are one the parent of the other and that they could contain the same set of vertices. Denote by $\Sigma_{3}$ the subsequence of $\Sigma$ that starts at $\sigma\left(u_{1}\right)$ and ends at $\sigma\left(u_{r}\right)$. We prove that $C_{1,2}$ is an outerclustered graph linearlyordered according to $\Sigma$ and that $C_{3}$ is an outerclustered graph linearly-ordered according to $\Sigma_{3}$.

Each cluster $\mu_{i}$ that is not crossed by path $\left(u_{1}, u_{2}, \ldots, u_{x}, \ldots, u_{r}\right)$ has two intersections with $\mathcal{C}_{1,2}$ and none with $\mathcal{C}_{3}$. Consider any cluster $\mu_{i}$ that is crossed by path $\left(u_{1}, u_{2}, \ldots, u_{x}, \ldots, u_{r}\right)$. Since $C$ satisfies Property $\mathrm{O} 2$, the boundary $B\left(\mu_{i}\right)$ of each cluster $\mu_{i}$ intersects $\mathcal{C}$ exactly twice. One of such intersections belongs to $\mathcal{C}_{1,2}$, the other one to $\mathcal{C}_{3}$. Hence, each of $\mathcal{C}_{1,2}$ and $\mathcal{C}_{3}$ crosses $B\left(\mu_{i}\right)$ exactly twice. Then, Lemma 1 ensures that $C_{1,2}$ and $C_{3}$ are biconnected internally-triangulated outerclustered graphs.

We prove that $C_{1,2}$ and $C_{3}$ satisfy Property LO2 of Definition 2. Path $\mathcal{P}_{1}$ and the path obtained by replacing the subpath of $\mathcal{P}_{2}$ between $u_{1}$ and $u_{r}$ with path $\left(u_{1}, u_{2}, \ldots, u_{x}, \ldots, u_{r}\right)$ are monotone paths delimiting $o\left(G_{1,2}\right)$. Further, path $\left(u_{1}, u_{2}, \ldots, u_{x}, \ldots, u_{r}\right)$ and the subpath of $\mathcal{P}_{2}$ between $u_{1}$ and $u_{r}$ are monotone paths delimiting $o\left(G_{3}\right)$.

We prove that $C_{1,2}$ and $C_{3}$ satisfy Property LO1. Since $C$ is linearly-ordered, the smallest cluster containing each vertex in $G$ (and each vertex in $G_{1,2}$ ) is a cluster $\mu_{i}$, that by hypothesis belongs to $\Sigma$. Concerning $C_{3}$, observe that $o\left(G_{3}\right)$ is delimited by two monotone paths. Hence, if the smallest cluster containing a vertex in $G_{3}$ does not belong to $\Sigma_{3}$, then such a vertex is outside $o\left(G_{3}\right)$, a contradiction.

We prove that $C_{1,2}$ and $C_{3}$ satisfy Property LO3. Sequence $\Sigma$ for $C_{1,2}$ coincides with sequence $\Sigma$ for $C$, hence the property follows. Concerning $C_{3}$, observe that the 
existence of an index $h$ such that (i) $\mu_{i+1}$ is the parent of $\mu_{i}$, for each $i<h$ such that $\mu_{i}$ belongs to $\Sigma_{3}$, and (ii) $\mu_{i+1}$ is a child of $\mu_{i}$, for each $i \geq h$ such that $\mu_{i+1}$ belongs to $\Sigma_{3}$, easily descends from the existence of index $h$ for the sequence $\Sigma$ of $C$ and from the fact that $\Sigma_{3}$ is a subsequence of $\Sigma$.

Now we turn our attention to the geometry supporting the above topological results.

Lemma 10 The drawings of $\left(\mathcal{C}_{1}, \mathcal{T}_{1}\right),\left(\mathcal{C}_{2}, \mathcal{T}_{2}\right)$, and $\left(\mathcal{C}_{3}, \mathcal{T}_{3}\right)$ in $\Gamma$ are convexseparated drawings.

Proof Drawing $\Gamma$ is straight-line and rectangular by construction. By construction, $u_{x}$ lies in $\operatorname{int}\left(T\left(v_{x}, u_{1}, u_{r}\right)\right)$ that in turn is entirely contained in $\operatorname{int}(P)$. By the convexity of $P$, straight-line segments can be drawn from $u_{x}$ to any vertex of $P$ (and hence to $u_{1}, v_{x}$, and $u_{r}$ ) not causing crossings; hence, the drawings of $\mathcal{C}_{1}, \mathcal{C}_{2}$, and $\mathcal{C}_{3}$ have no edge crossings. Since $\Gamma\left(C_{o}\right)$ has no region-region crossings, $\Gamma$ has no region-region crossings (namely, each cluster has the same drawing in $\Gamma$ and in $\Gamma\left(C_{o}\right)$ ).

We prove that $\Gamma$ has no edge-region crossing. Suppose that there is an edgeregion crossing between an edge $e$ and a cluster $v$. Then, $e$ is either an edge of path $\left(u_{1}, u_{2}, \ldots, u_{x}\right)$, or an edge of path $\left(u_{x}, u_{x+1}, \ldots, u_{r}\right)$, or edge $\left(u_{x}, v_{x}\right)$. Namely, all other edge-cluster pairs have the same drawings in $\Gamma$ and in $\Gamma\left(C_{o}\right)$, hence they do not cross more than once by hypothesis. Suppose that $e=\left(u_{i}, u_{i+1}\right)$ is an edge of $\left(u_{1}, u_{2}, \ldots, u_{x}\right)$, the other cases being analogous. If both $u_{i}$ and $u_{i+1}$ belong to $v$ then, by the convexity of $v, e$ is internal to $v$. If exactly one of $u_{i}$ and $u_{i+1}$ belongs to $v$ then, by the convexity of $v, e$ crosses $v$ exactly once. It follows that $v$ contains neither $u_{i}$ nor $u_{i+1}$. Consider the parent $\mu$ of $v$ in $T$. Such a parent exists since otherwise $v$ would be the root of $T$, contradicting the fact that $v$ contains neither $u_{i}$ nor $u_{i+1}$. By definition of convex-separated drawing, there exists a convex region $R(\mu, v)$ with the properties described in Definition 3 which separates $v$ from the rest of the drawing, thus avoiding an edge-region crossing between $e$ and $v$. More precisely, by definition of outerclustered graph, $v$ has exactly two incident edges $e_{1}(v)$ and $e_{2}(v)$ belonging to $o(G)$. Denote by $u\left(e_{1}(v)\right)$ and $u\left(e_{2}(v)\right)$ the end-vertices of $e_{1}(v)$ and $e_{2}(v)$ belonging to $v$. Denote by $p\left(l_{1}\right)$ the endpoint of $l_{1}(\mu, v)$ that lies on $e_{1}(v)$ (if both endpoints of $l_{1}(\mu, v)$ lie on $e_{1}(v)$, then $p\left(l_{1}\right)$ is the one that is closer to $\left.u\left(e_{1}(v)\right)\right)$. Note that an endpoint of $l_{1}(\mu, v)$ lying on $e_{1}(\nu)$ exists as $\Gamma$ satisfies Property CS3. Analogously define $p\left(l_{2}\right)$. Then, segment $\overline{p\left(l_{1}\right) p\left(l_{2}\right)}$ splits $P$ into two disjoint convex polygons $P^{\prime}$ and $P^{\prime \prime}$, such that, out of $o(G)$ and of path $\left(u_{1}, u_{2}, \ldots, u_{x}\right), P^{\prime}$ contains all and only the vertices in $v$ and $P^{\prime \prime}$ contains all and only the vertices not in $v$, as $\Gamma$ satisfies Property CS3. By the convexity of $P^{\prime}$ and $P^{\prime \prime}, e$ is internal to $P^{\prime \prime}$, while the part of $v$ inside $P$ is internal to $P^{\prime}$. Hence, $e$ does not cross $v$.

We prove that $\Gamma$ satisfies Property CS1 of Definition 3. Denote by $P_{i}$ the polygon representing $\mathcal{C}_{i}$ in $\Gamma$, for each $i \in\{1,2,3\}$. Every angle that is incident to a vertex in $\mathcal{C}$ different from $u_{1}, u_{r}$, and $v_{x}$ in $P_{1}, P_{2}$, or $P_{3}$ is no more than $180^{\circ}$, since it is the same angle as in $P$. Every angle that is incident to a vertex in the path $\left(u_{1}, u_{2}, \ldots, u_{r}\right)$ different from $u_{1}, u_{x}$, and $u_{r}$ in $P_{1}, P_{2}$, or $P_{3}$ is exactly $180^{\circ}$, by construction. Every angle incident to $u_{1}, v_{x}$, and $u_{r}$ in $P_{1}, P_{2}$, or $P_{3}$ is strictly less than $180^{\circ}$, since it 
is strictly less than an angle that is at most $180^{\circ}$ (namely the angle incident to the same vertex in $P$ ); finally, the three angles $\widehat{u_{1} u_{x} v_{x}}, \widehat{u_{1} u_{x} u_{r}}$, and $\widehat{u_{r} u_{x} v_{x}}$ incident to $u_{x}$ are all strictly less than $180^{\circ}$, since they are angles in the non-degenerate triangles $T\left(u_{1} u_{x} v_{x}\right), T\left(u_{1} u_{x} u_{r}\right)$, and $T\left(u_{r} u_{x} v_{x}\right)$, respectively.

We prove that $\Gamma$ satisfies Property CS2. Concerning the drawing of $\mathcal{C}_{1}$ (the arguments for the drawing of $\mathcal{C}_{2}$ being analogous), observe that $C_{1}$ is linearly-ordered according to the subsequence $\Sigma_{1}$ of $\Sigma$ that starts at $\sigma\left(v_{i}\right)=\mu_{1}$ and ends at the one of $\sigma\left(u_{x}\right)$ and $\sigma\left(v_{x}\right)$ that comes after in $\Sigma$. The angle incident to $v_{i}$ in $P_{1}$ is strictly less than $180^{\circ}$, by hypothesis; further, as proved above, the angles incident to $u_{x}$ and $v_{x}$ in $P_{1}$ are strictly less than $180^{\circ}$. Concerning the drawing of $\mathcal{C}_{3}$, observe that $\sigma\left(u_{1}\right)$ and $\sigma\left(u_{r}\right)$ are the first and the last cluster in $\Sigma_{3}$. Further, the angles incident to $u_{1}$ and to $u_{r}$ in $P_{3}$ are strictly smaller than $180^{\circ}$, as proved above.

We prove that $\Gamma$ satisfies Property CS3. For each $i=1,2,3$, let $\mu_{j}$ be any cluster (except for the first and the last one) belonging to the sequence according to which $C_{i}$ is linearly-ordered and let $\mu_{j}^{\prime}$ be any child of $\mu_{j}$. The existence of a region $R\left(\mu_{j}, \mu_{j}^{\prime}\right)$ inside $P_{i}$ is easily deduced from the existence of region $R\left(\mu_{j}, \mu_{j}^{\prime}\right)$ inside $P$. Namely, either $R\left(\mu_{j}, \mu_{j}^{\prime}\right)$ inside $P$ is intersected neither by path $\left(u_{1}, u_{2}, \ldots, u_{r}\right)$ nor by edge $\left(u_{x}, v_{x}\right)$, implying that a region $R\left(\mu_{j}, \mu_{j}^{\prime}\right)$ inside $P_{i}$ can be constructed coincident with the same region inside $P$, or $R\left(\mu_{j}, \mu_{j}^{\prime}\right)$ inside $P$ is intersected by one or both of path $\left(u_{1}, u_{2}, \ldots, u_{r}\right)$ and edge $\left(u_{x}, v_{x}\right)$, that thus split $R\left(\mu_{j}, \mu_{j}^{\prime}\right)$ into two or three regions inside the polygons $P_{i}$. The properties that have to be satisfied by $R\left(\mu_{j}, \mu_{j}^{\prime}\right)$ inside $P_{i}$ easily descend from the analogous properties satisfied by $R\left(\mu_{j}, \mu_{j}^{\prime}\right)$ inside $P$.

Graphs $C_{1}, C_{2}$, and $C_{3}$ are, in general, not triconnected; namely, there could exist chords between any vertex in $\left(u_{1}, u_{2}, \ldots, u_{x-1}\right)$ and any vertex in $\left(u_{x+1}, u_{x+2}\right.$, $\left.\ldots, u_{r}\right)$, or chords between any vertex in $\left(u_{1}, u_{2}, \ldots, u_{r}\right)$ and any vertex in the path of $\mathcal{C}$ connecting $u_{1}$ and $u_{r}$ and not containing $v_{x}$, or chords between $u_{x}$ and any vertex in the path of $\mathcal{C}$ connecting $u_{1}$ and $u_{r}$ and containing $v_{x}$. By Lemma 2, each of these chords splits a linearly-ordered outerclustered graph into two smaller linearly-ordered outerclustered graphs. Further, by construction the end-vertices of each of such chords are not collinear with any other vertex of the cycle. Hence, by Lemma 3, drawing the chords as straight-line segments splits the drawings of $C_{1}$, $C_{2}$, and $C_{3}$, that are convex-separated by Lemma 10, into convex-separated drawings. When all the chords have been added, the underlying graphs of the resulting internally-triangulated linearly-ordered outerclustered graphs are all triconnected. Hence, the induction applies and an internally-convex-separated drawing of each of such linearly-ordered clustered graphs can be constructed inside the corresponding outer face, thus obtaining an internally-convex-separated drawing of $C$.

Case 2 applies when the vertices of $V_{2} \cup\left\{v_{i}, v_{j}\right\}$ are not all collinear. In such a case, $V_{2} \neq \emptyset$ and a path $\left(u_{1}, u_{2}, \ldots, u_{x}, \ldots, u_{r}\right)$ can be found as in Lemma 6 . Analogously to Case 1, $C$ is decomposed into smaller triconnected internally-triangulated linearly-ordered outerclustered graphs; path $\left(u_{1}, u_{2}, \ldots, u_{x}, \ldots, u_{r}\right)$ and all the edges connecting vertices of such a path with vertices of $\mathcal{C}$ can be drawn so that the outer faces of the corresponding outerclustered graphs are represented by convex-separated 


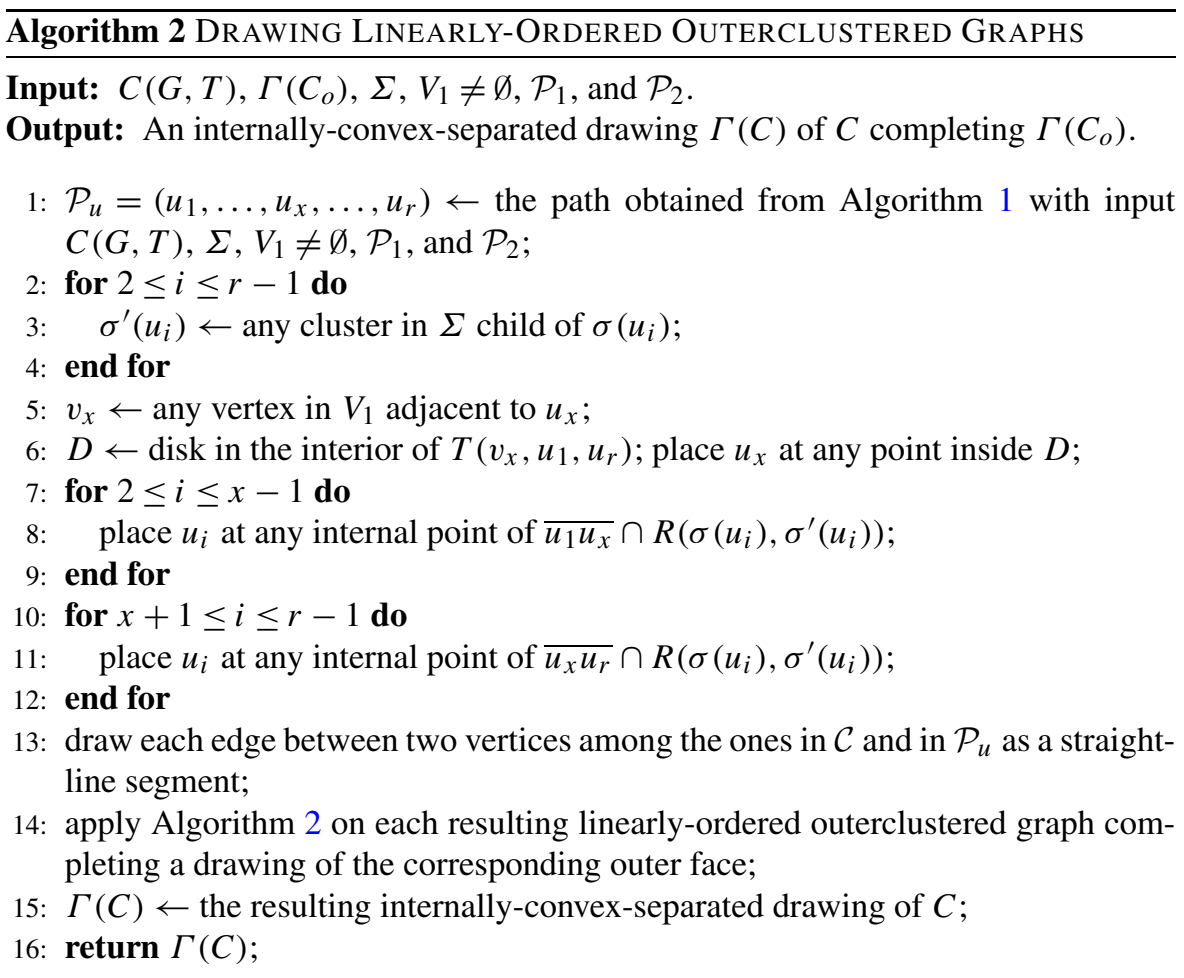

drawings. Hence, the induction applies and an internally-convex-separated drawing of each of such linearly-ordered clustered graphs can be constructed inside the corresponding outer face, thus obtaining an internally-convex-separated drawing of $C$.

A pseudo-code description of the algorithm for drawing a linearly-ordered outerclustered graph $C$ (supposing that $V_{1} \neq \emptyset$ ) is presented in Algorithm 2.

It remains to prove that one out of Case 1 and Case 2 applies. If Case 1 does not apply, then the vertices of $V_{1} \cup\left\{v_{i}, v_{j}\right\}$ are collinear, and if Case 2 does not apply, then the vertices of $V_{2} \cup\left\{v_{i}, v_{j}\right\}$ are collinear. However, this implies that all the vertices of $\mathcal{C}$ are collinear, contradicting the fact that $\Gamma\left(C_{o}\right)$ is a convex-separated drawing. This concludes the proof of Theorem 1.

\section{Drawing Maximal Outerclustered Graphs}

In this section we show how to draw maximal outerclustered graphs, exploiting the results obtained on linearly-ordered outerclustered graphs. Namely, in order to show that any maximal outerclustered graph has an internally-convex-separated drawing completing an arbitrary triangular-convex-separated drawing of its outer face, we show how to reduce the problem of drawing a maximal outerclustered graph to the one of drawing some linearly-ordered outerclustered graphs.

First, we need the following preliminary results. 
Fig. 17 Illustration for the proof of Lemma 11

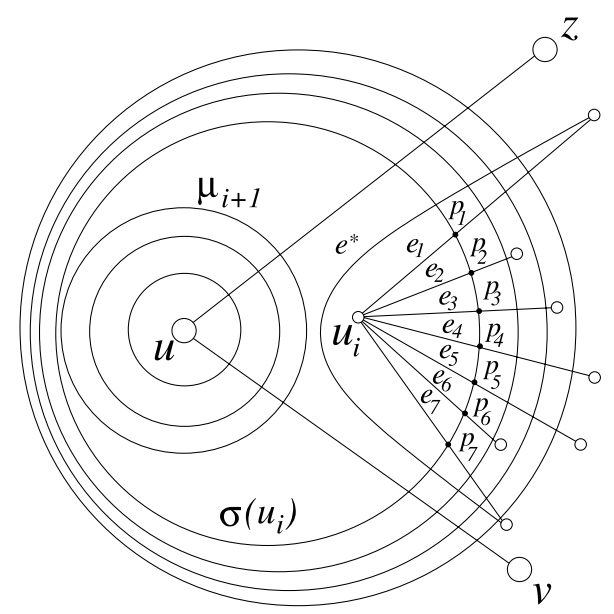

Lemma 11 Let $C(G, T)$ be a maximal outerclustered graph and let $u, v$, and $z$ be the vertices incident to $o(G)$. Let $u_{i} \neq u$ be any internal vertex of $G$ such that $\sigma\left(u_{i}\right)$ contains $u$ and contains neither $v$ nor $z$. Then, there exists an edge $\left(u_{i}, u_{i+1}\right)$ in $G$ such that $\sigma\left(u_{i+1}\right)$ is a descendant of $\sigma\left(u_{i}\right)$.

Proof Refer to Fig. 17. Let $\mu_{i+1}$ be the only cluster child of $\sigma\left(u_{i}\right)$ and containing $u$. Notice that such a child exists. Namely, if such a child does not exist, then $\sigma\left(u_{i}\right)=$ $\sigma(u)$; however, this would imply that $u_{i}=u$, since $\sigma(u)$ induces a connected graph, by the $c$-planarity and the maximality of $C$, and since an outerclustered graph does not contain two adjacent vertices having the same smallest containing cluster if one of them is internal, by Property O3 of Definition 1. Suppose, for a contradiction, that $u_{i}$ is not adjacent to any vertex in $\mu_{i+1}$. By Property O3, $u_{i}$ is not adjacent to any vertex $v_{j}$ such that $\sigma\left(v_{j}\right)=\sigma\left(u_{i}\right)$. It follows that each edge $e_{l}$ incident to $u_{i}$ intersects the boundary $B\left(\sigma\left(u_{i}\right)\right)$ of $\sigma\left(u_{i}\right)$ in a point $p_{l}$ belonging to the polygonal line $l\left(\sigma\left(u_{i}\right)\right)$ that is the part of $B\left(\sigma\left(u_{i}\right)\right)$ lying in the interior of $o(G)$. Order the points $p_{l}$ as they are encountered walking on $l\left(\sigma\left(u_{i}\right)\right)$ from one endpoint to the other one. Denote by $p_{1}, p_{2}, \ldots, p_{m}$ such points. By the maximality of $G$ and by the fact that $G$ contains internal vertices, every vertex of $G$ has degree at least three. Hence, $m \geq 3$ and there exists an internal face delimited by a 3 -cycle containing edges $e_{1}$ and $e_{m}$. However, the third edge $e^{*}$ of such a cycle crosses twice $l\left(\sigma\left(u_{i}\right)\right)$ and hence $B\left(\sigma\left(u_{i}\right)\right)$, contradicting the $c$-planarity of the considered embedding.

Corollary 1 Let $C(G, T)$ be any maximal outerclustered graph and let $u, v$, and $z$ be the vertices incident to $o(G)$. Suppose that $\sigma(u) \neq \sigma(v), \sigma(z)$. Let $u_{1}$ be any internal vertex of $G$ such that $\sigma\left(u_{1}\right)$ contains $u$ and contains neither $v$ nor $z$. Then, there exists a chordless path $\left(u_{1}, u_{2}, \ldots, u_{k}\right)$ in $G$ such that $u_{k}=u$ and such that $\sigma\left(u_{i+1}\right)$ is a descendant of $\sigma\left(u_{i}\right)$, for each $i=1,2, \ldots, k-1$.

Proof Suppose that a path $\left(u_{1}, u_{2}, \ldots, u_{i}\right)$ has already been determined such that $u_{j+1}$ is a descendant of $u_{j}$, for each $j=1,2, \ldots, i-1$. By Lemma 11 , there exists 
Algorithm 3 ChORDLESS PATHS IN MAXIMAL OUTERCLUSTERED GRAPHS

Input: $C(G, T), u$, and $u_{1}$, as in Corollary 1 .

Output: A path $\left(u_{1}, u_{2}, \ldots, u_{k}\right)$ such that $u_{k}=u$ and such that $\sigma\left(u_{i+1}\right)$ is a descendant of $\sigma\left(u_{i}\right)$.

1: $u_{j} \leftarrow u_{1}$

2: while $u_{j} \neq u$ do

3: $\quad u_{i} \leftarrow u_{j}$;

4: $\quad\left(u_{i}, u_{i+1}\right) \leftarrow$ an edge in $G$ such that $\sigma\left(u_{i+1}\right)$ is a descendant of $\sigma\left(u_{i}\right)$;

5: $\quad$ add $\left(u_{i}, u_{i+1}\right)$ to the current path;

6: $\quad u_{j} \leftarrow u_{i+1}$;

7: end while

8: $\left(u_{1}, u_{2}, \ldots, u_{k}\right) \leftarrow$ the path obtained by Algorithm 4 on $G$ and the current path;

9: return $\left(u_{1}, u_{2}, \ldots, u_{k}\right)$;

\section{Algorithm 4 RePlaCE CHORDS IN PATHS}

Input: A graph $G$ and a path $\left(u_{1}, u_{2}, \ldots, u_{k}\right)$ in $G$.

Output: A chordless path in $G$ whose vertices are a subset of $\left\{u_{1}, u_{2}, \ldots, u_{k}\right\}$ in the same order as in $\left(u_{1}, u_{2}, \ldots, u_{k}\right)$.

1: while the current path $\left(u_{1}, u_{2}, \ldots, u_{k}\right)$ has a chord $\left(u_{i}, u_{j}\right)$ in $G$ do

2: $\quad$ replace the subpath of $\left(u_{1}, u_{2}, \ldots, u_{k}\right)$ between $u_{i}$ and $u_{j}$ with edge $\left(u_{i}, u_{j}\right)$;

3: end while

4: return the obtained path;

an edge $\left(u_{i}, u_{i+1}\right)$ in $G$ such that $\sigma\left(u_{i+1}\right)$ is a descendant of $\sigma\left(u_{i}\right)$. Repeating such an argument eventually leads to choose a vertex $u_{k}$ in $\sigma(u)$. Since $\sigma(u) \neq \sigma(v), \sigma(z)$, then, by Property O3 of Definition $1, u$ is the only vertex in $\sigma(u)$. It follows that $u_{k}=u$. The obtained path $\mathcal{P}_{u}$ may have chords. Suppose that the currently considered path $\mathcal{P}_{u}^{l}=\left(u_{1}^{l}, u_{2}^{l}, \ldots, u_{k}^{l}\right)$ has a chord $\left(u_{i}^{l}, u_{j}^{l}\right)$, with $j>i+1$ and with $\mathcal{P}_{u}^{1}=\mathcal{P}_{u}$. Obtain a new path $\mathcal{P}_{u}^{l+1}$ by replacing the subpath $\left(u_{i}^{l}, u_{i+1}^{l}, \ldots, u_{j}^{l}\right)$ of $\mathcal{P}_{u}^{l}$ with the chord $\left(u_{i}^{l}, u_{j}^{l}\right)$. Clearly, $\sigma\left(u_{j}^{l}\right)$ is a descendant of $\sigma\left(u_{i}^{l}\right)$. Further, $\mathcal{P}_{u}^{l+1}$ has at least one chord less than $\mathcal{P}_{u}^{l}$ which implies that, after a certain number of steps, the current path is chordless.

A pseudo-code description of the algorithm for finding a path satisfying the requirements of Corollary 1 in a maximal outerclustered graph is presented in Algorithms 3-4.

Let $C(G, T)$ be a maximal outerclustered graph and let $u, v$, and $z$ be the vertices incident to $o(G)$. Suppose that: (i) $\sigma(u) \neq \sigma(v), \sigma(u) \neq \sigma(z)$, and $\sigma(v) \neq \sigma(z)$; (ii) if there exists a cluster containing exactly two vertices incident to $o(G)$, then such vertices are $u$ and $v$; (iii) if the smallest containing cluster of one of $u$ and $v$ contains the other one, then $\sigma(v)$ contains $u$; and (iv) $G$ has internal vertices. The following lemma states that in a maximal outerclustered graph some edge-disjoint 


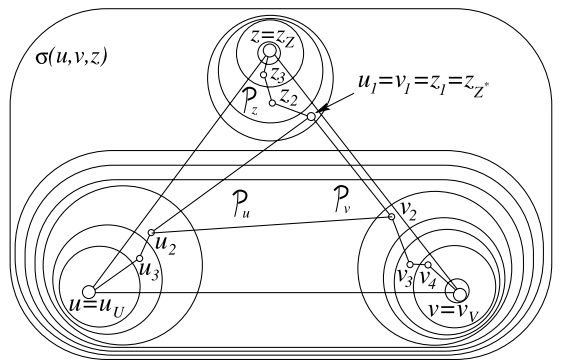

(a)

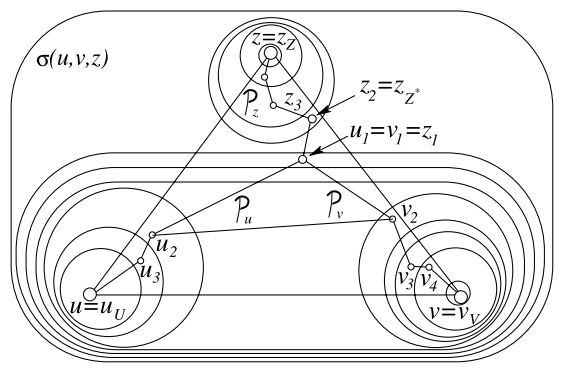

(c)

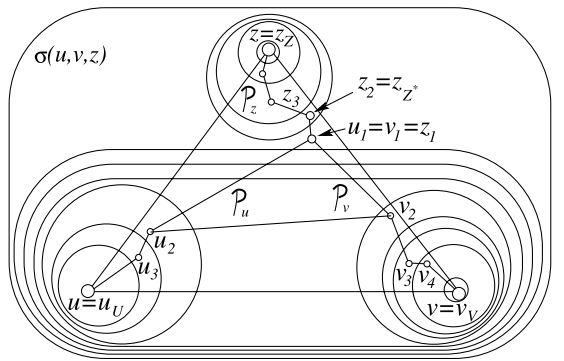

(b)

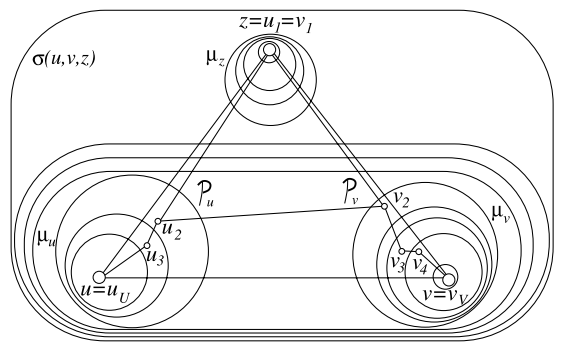

(d)

Fig. 18 (a)-(c) Three paths satisfying Condition 1 of Lemma 12. In (a) $\sigma\left(z_{1}\right)$ is a cluster containing $z$ and not containing $u$ and $v$; in (b) $\sigma\left(z_{1}\right)=\sigma(u, v, z)$; in (c) $\sigma\left(z_{1}\right)$ is a cluster not containing $z$ and containing $u$ and $v$. (d) Two paths satisfying Condition 2 of Lemma 12

paths can be found satisfying certain properties of "monotonicity" with respect to the cluster hierarchy. After the lemma, it will be shown how such paths, together with their chords, split $C$ into linearly-ordered outerclustered graphs.

Lemma 12 One of the following holds:

1. There exist three paths $\mathcal{P}_{u}=\left(u_{1}, u_{2}, \ldots, u_{U}\right), \mathcal{P}_{v}=\left(v_{1}, v_{2}, \ldots, v_{V}\right)$, and $\mathcal{P}_{z}=$ $\left(z_{1}, z_{2}, \ldots, z_{Z}\right)$ such that (see Figs. 18(a)-18(c)):

(a) $u_{U}=u, v_{V}=v, z_{Z}=z$, and $u_{1}=v_{1}=z_{1}$;

(b) the vertices of $\mathcal{P}_{u} \backslash\left\{u_{1}\right\}, \mathcal{P}_{v} \backslash\left\{v_{1}\right\}$, and $\mathcal{P}_{z} \backslash\left\{z_{1}\right\}$ are distinct;

(c) each of paths $\mathcal{P}_{u} \backslash\left\{u_{1}\right\}, \mathcal{P}_{v} \backslash\left\{v_{1}\right\}$, and $\mathcal{P}_{z}$ has no chords;

(d) $\sigma\left(u_{i}\right)$ contains neither $v$ nor $z$, for each $2 \leq i \leq U ; \sigma\left(v_{i}\right)$ contains neither $u$ nor $z$, for each $2 \leq i \leq V ; \sigma\left(z_{i}\right)$ contains neither $u$ nor $v$, for each $Z^{*} \leq i \leq$ $Z$, where $Z^{*}$ is an index such that $1 \leq Z^{*} \leq Z$;

(e) $\sigma\left(u_{i+1}\right)$ is a descendant of $\sigma\left(u_{i}\right)$, for each $2 \leq i \leq U-1 ; \sigma\left(v_{i+1}\right)$ is a descendant of $\sigma\left(v_{i}\right)$, for each $2 \leq i \leq V-1$;

(f) $\sigma\left(z_{1}\right)$ is either a cluster containing $z$ and not containing $u$ and $v$ (then $Z^{*}=1$ and $\sigma\left(z_{i+1}\right)$ is a descendant of $\sigma\left(z_{i}\right)$, for each $\left.1 \leq i \leq Z-1\right)$, or $\sigma(u, v, z)$ (then $Z^{*}=2$ and $\sigma\left(z_{i+1}\right)$ is a descendant of $\sigma\left(z_{i}\right)$, for each $1 \leq i \leq Z-1$ ), or a cluster not containing $z$ and containing $u$ and $v$. In the latter case $Z^{*} \geq 2$, $\sigma\left(z_{i+1}\right)$ is an ancestor of $\sigma\left(z_{i}\right)$, for each $1 \leq i \leq Z^{*}-2, \sigma\left(z_{i+1}\right)$ is a descendant of $\sigma\left(z_{i}\right)$, for each $Z^{*} \leq i \leq Z-1$, and either $\sigma\left(z_{Z^{*}}\right)$ is a descendant 
of $\sigma\left(z_{Z^{*}-1}\right)$ (if $\left.\sigma\left(z_{Z^{*}-1}\right)=\sigma(u, v, z)\right)$, or $\sigma\left(z_{Z^{*}}\right)$ is not comparable with $\sigma\left(z Z^{*-1}\right)$ (if $\sigma\left(z_{Z^{*}-1}\right)$ contains $u$ and $v$ and does not contain $\left.z\right)$;

(g) contains an internal face having incident vertices $u_{1}, u_{2}$, and $v_{2}$.

2. There exist two paths $\mathcal{P}_{u}=\left(u_{1}, u_{2}, \ldots, u_{U}\right)$ and $\mathcal{P}_{v}=\left(v_{1}, v_{2}, \ldots, v_{V}\right)$ such that (see Fig. 18(d)):

(a) $u_{U}=u, v_{V}=v$, and $u_{1}=v_{1}=z$;

(b) the vertices of $\mathcal{P}_{u} \backslash\left\{u_{1}\right\}$ and $\mathcal{P}_{v} \backslash\left\{v_{1}\right\}$ are distinct;

(c) each of paths $\mathcal{P}_{u} \backslash\left\{u_{1}\right\}$ and $\mathcal{P}_{v} \backslash\left\{v_{1}\right\}$ has no chords;

(d) $\sigma\left(u_{i}\right)$ contains neither $v$ nor $z$, for each $2 \leq i \leq U$; $\sigma\left(v_{i}\right)$ contains neither $u$ nor $z$, for each $2 \leq i \leq V$;

(e) $\sigma\left(u_{i+1}\right)$ is a descendant of $\sigma\left(u_{i}\right)$, for each $2 \leq i \leq U-1 ; \sigma\left(v_{i+1}\right)$ is a descendant of $\sigma\left(v_{i}\right)$, for each $2 \leq i \leq V-1$;

(f) $G$ contains an internal face having incident vertices $u_{2}, v_{2}$, and $z$.

Proof Consider the biggest cluster $\mu_{u}$ containing $u$ and not containing $v$. Notice that such a cluster exists. Namely, $\sigma(u)$ does not contain $v$ by hypothesis. Consider the biggest cluster $\mu_{v}$ containing $v$ and not containing $u$, if any such a cluster exists. If $\mu_{v}$ exists, let $E^{\prime}$ be the set of edges whose end-vertices are one in $\mu_{u}$ and one in $\mu_{v}$. If $\mu_{v}$ does not exist, let $E^{\prime}$ be the set of edges incident to $v$ whose other end-vertex is in $\mu_{u}$. In both cases, there exists at least one of such edges, in fact $(u, v)$. Order the edges in $E^{\prime}$ as they are encountered when walking on the part $l\left(\mu_{u}\right)$ of the boundary $B\left(\mu_{u}\right)$ of $\mu_{u}$ that is internal to $G$, starting from the intersection of $(u, v)$ with $B\left(\mu_{u}\right)$. See Fig. 19.

Consider the last edge $\left(u^{\prime}, v^{\prime}\right)$ in $E^{\prime}$ and consider the internal face $\left(u^{\prime}, v^{\prime}, z^{\prime}\right)$ such that $\left(u^{\prime}, z^{\prime}\right)$ is the edge following $\left(u^{\prime}, v^{\prime}\right)$ in the order in which the edges incident to $\mu_{u}$ are encountered when walking on the part of $B\left(\mu_{u}\right)$ that is internal to $G$, starting from the intersection of $(u, v)$ with $B\left(\mu_{u}\right)$. Let $\mathcal{P}_{u}=\left(u_{1}, u_{2}, \ldots, u_{U}\right)$ be a path such that $u_{1}=z^{\prime}, u_{2}=u^{\prime}$, and $\left(u_{2}, u_{3}, \ldots, u_{U}\right)$ is a chordless path such that $u_{U}=u$ and $\sigma\left(u_{i+1}\right)$ is a descendant of $\sigma\left(u_{i}\right)$, for $2 \leq i \leq U-1$. Such a path exists by Corollary 1 (notice that $\sigma\left(u_{2}\right)$ contains $u$ and contains neither $v$ nor $z$ ). Further, let $\mathcal{P}_{v}=\left(v_{1}, v_{2}, \ldots, v_{V}\right)$ be a path such that $v_{1}=z^{\prime}, v_{2}=v^{\prime}$, and $\left(v_{2}, v_{3}, \ldots, v_{V}\right)$ is a chordless path such that $v_{V}=v$ and $\sigma\left(v_{i+1}\right)$ is a descendant of $\sigma\left(v_{i}\right)$, for $2 \leq i \leq$

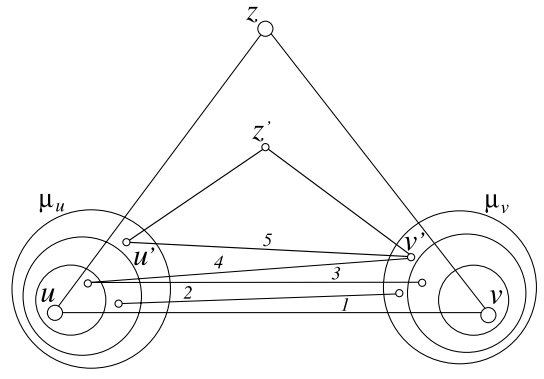

(a)

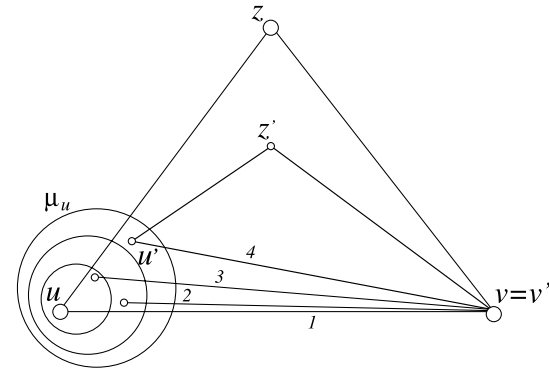

(b)

Fig. 19 The edges in $E^{\prime}$. The numbers on such edges indicate the order in which such edges are encountered when walking on the part of $B\left(\mu_{u}\right)$ internal to $G$, starting from the intersection of $(u, v)$ with $B\left(\mu_{u}\right)$. (a) $\mu_{v}$ exists. (b) $\mu_{v}$ does not exist 


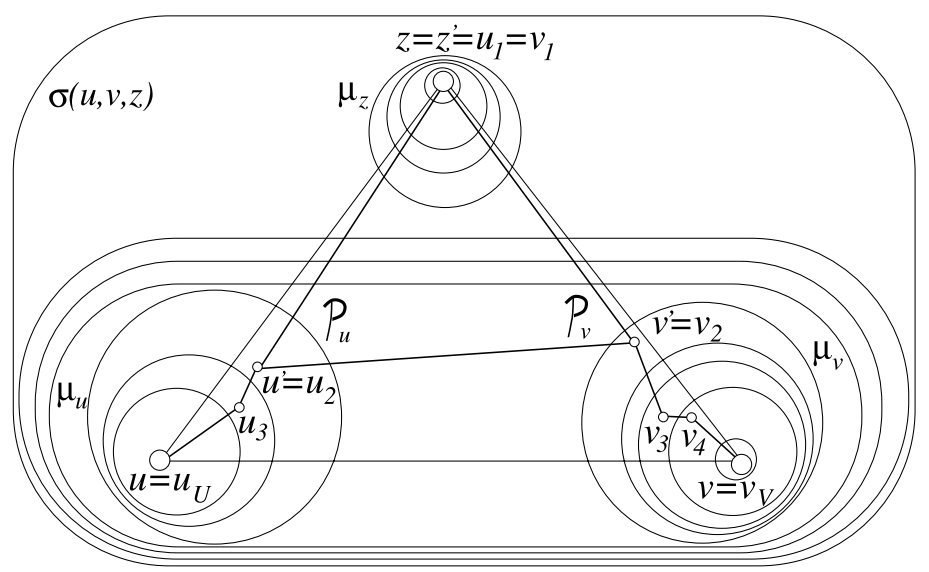

Fig. 20 If $z^{\prime}=z$, then paths $\mathcal{P}_{u}$ and $\mathcal{P}_{v}$ satisfy Condition 2 of Lemma 12

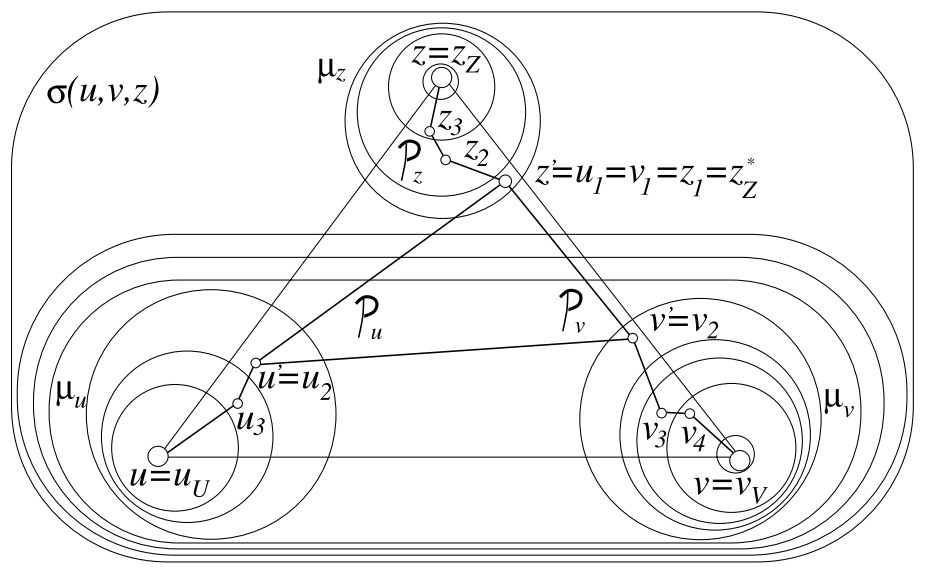

Fig. 21 If $z^{\prime} \neq z$ and $\sigma\left(z^{\prime}\right)$ contains $z$ and contains neither $u$ nor $v$, then paths $\mathcal{P}_{u}$, $\mathcal{P}_{v}$, and $\mathcal{P}_{z}$ satisfy Condition 1 of Lemma 12

$V-1$. Notice that if $\mu_{v}$ exists, then such a path exists by Corollary 1 (notice that $\sigma\left(v_{2}\right)$ contains $v$ and contains neither $u$ nor $z$ ). Otherwise, $v_{2}=v_{V}=v$.

Suppose that $z^{\prime}=z$ (see Fig. 20). Then, $G$ contains an internal face having incident vertices $u_{2}, v_{2}$, and $z$. By construction, paths $\mathcal{P}_{u}$ and $\mathcal{P}_{v}$ satisfy Condition 2 of the lemma.

Suppose that $z \neq z^{\prime}$. Vertex $z^{\prime}$ belongs neither to $\mu_{u}$ nor to $\mu_{v}$, otherwise edge $\left(z^{\prime}, v^{\prime}\right)$ or edge $\left(z^{\prime}, u^{\prime}\right)$ would follow $\left(u^{\prime}, v^{\prime}\right)$ in $E^{\prime}$, a contradiction. Hence, $\sigma\left(z^{\prime}\right)$ is either a cluster containing $z$ and not containing $u$ and $v$, or is $\sigma(u, v, z)$, or is a cluster containing $u$ and $v$ and not containing $z$.

- Suppose that $\sigma\left(z^{\prime}\right)$ contains $z$ and contains neither $u$ nor $v$ (see Fig. 21). Let $\mathcal{P}_{z}=$ $\left(z_{1}, z_{2}, \ldots, z_{Z}\right)$ be a chordless path such that $z_{1}=z^{\prime}, z_{Z}=z$, and $\sigma\left(z_{i+1}\right)$ is a descendant of $\sigma\left(z_{i}\right)$, for $2 \leq i \leq Z-1$. Such a path exists by Corollary 1 (notice 


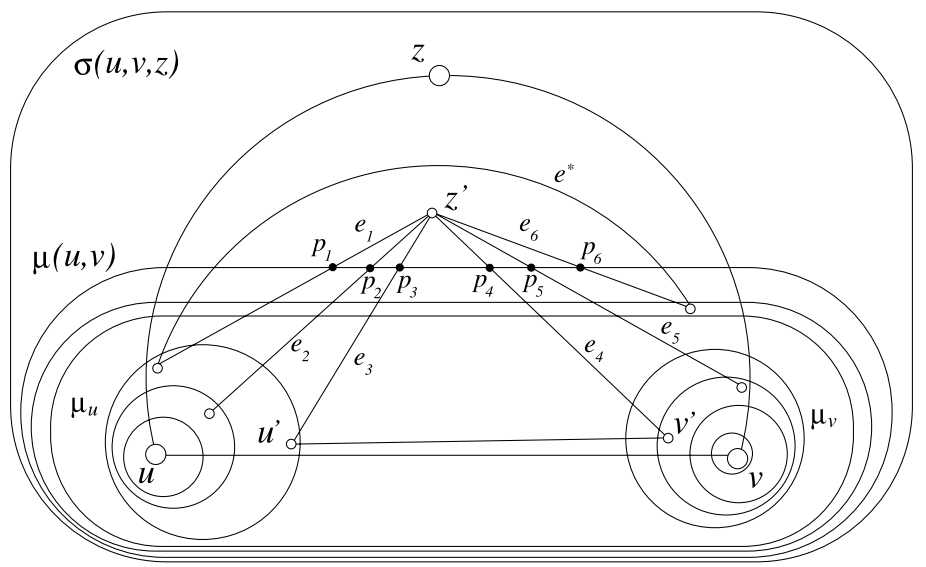

Fig. 22 If $z^{\prime} \neq z$, if $\sigma\left(z^{\prime}\right)=\sigma(u, v, z)$, and if a cluster containing both $u$ and $v$ and not containing $z$ exists, then $\mu_{z}$ exists

that $\sigma\left(z_{1}\right)$ contains $z$ and contains neither $u$ nor $v$ ). By construction, paths $\mathcal{P}_{u}, \mathcal{P}_{v}$, and $\mathcal{P}_{z}$ satisfy Condition 1 of the lemma.

- Suppose that $\sigma\left(z^{\prime}\right)=\sigma(u, v, z)$. Let $\mu_{z}$ be the biggest cluster containing $z$ and containing neither $u$ nor $v$.

We claim that $\mu_{z}$ exists. Suppose, for a contradiction, that $\mu_{z}$ does not exist. Then, every cluster containing $z$ also contains one of $u$ and $v$; therefore, any such a cluster contains both $u$ and $v$, by the assumptions of the lemma. Hence, $\sigma(z)=\sigma(u, v, z)=\sigma\left(z^{\prime}\right)$, and $\sigma(u, v, z)$ is the smallest containing cluster of at least two vertices, namely $z$ and $z^{\prime}$. We prove that there exists an edge between two vertices whose smallest containing cluster is $\sigma(z)$, thus contradicting the fact that $C$ satisfies Property O3, and hence proving that $\mu_{z}$ exists. Consider the edges incident to $z^{\prime}$.

First, suppose that a cluster containing both $u$ and $v$ and not containing $z$ exists (see Fig. 22). Let $\mu_{u, v}$ be the biggest cluster containing both $u$ and $v$ and not containing $z$. Then, if no neighbor of $z^{\prime}$ has $\sigma\left(z^{\prime}\right)$ as smallest containing cluster, each edge $e_{l}$ incident to $z^{\prime}$ intersects the boundary $B\left(\mu_{u, v}\right)$ of $\mu_{u, v}$ in a point $p_{l}$ belonging to the polygonal line $l\left(\mu_{u, v}\right)$ that is the part of $B\left(\mu_{u, v}\right)$ lying in the interior of $o(G)$. Order the points $p_{l}$ as they are encountered when walking on $l\left(\mu_{u, v}\right)$ from one endpoint to the other one. Denote by $p_{1}, p_{2}, \ldots, p_{m}$ such points. By the maximality of $G$ and by the fact that $G$ contains internal vertices, every vertex of $G$ has degree at least three. Hence, $m \geq 3$ and there exists a face delimited by a 3 -cycle containing edges $e_{1}$ and $e_{m}$. However, the third edge $e^{*}$ of such a cycle crosses twice $l\left(\mu_{u, v}\right)$ and hence $B\left(\mu_{u, v}\right)$, contradicting the $c$-planarity of the considered embedding. Second, suppose that a cluster containing both $u$ and $v$ and not containing $z$ does not exist (see Fig. 23). Then, if $\mu_{v}$ exists and if no neighbor of $z^{\prime}$ has $\sigma\left(z^{\prime}\right)$ as smallest containing cluster, each edge $e_{l}$ incident to $z^{\prime}$ is also incident either to a vertex belonging to $\mu_{u}$ or to a vertex belonging to $\mu_{v}$; if $\mu_{v}$ does not exist and if no neighbor of $z^{\prime}$ has $\sigma\left(z^{\prime}\right)$ as smallest containing cluster, each edge $e_{l}$ incident to $z^{\prime}$ is also incident to a vertex belonging to $\mu_{u}$. 


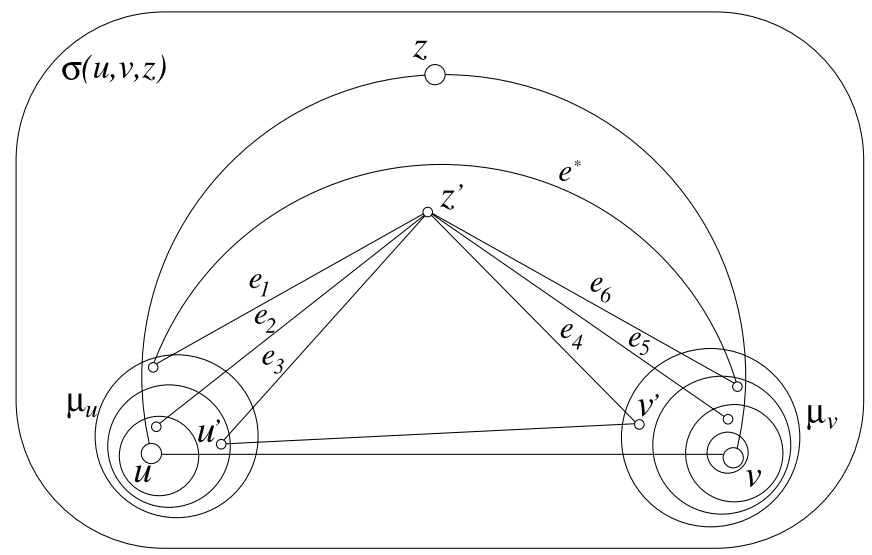

Fig. 23 If $z^{\prime} \neq z$, if $\sigma\left(z^{\prime}\right)=\sigma(u, v, z)$, and if a cluster containing both $u$ and $v$ and not containing $z$ does not exist, then $\mu_{z}$ exists

By the $c$-planarity of the embedding, the order of the edges incident to $z^{\prime}$ is a sequence $\left(e_{1}, e_{2}, \ldots, e_{i}, e_{i+1}, \ldots, e_{m}\right)$ so that: (i) $e_{j}$ is incident to a vertex in $\mu_{u}$, for $j=1,2, \ldots, i$, (ii) $e_{i}=\left(z^{\prime}, u^{\prime}\right)$, (iii) $e_{i+1}=\left(z^{\prime}, v^{\prime}\right)$, and (iv) $e_{j}$ is incident to a vertex in $\mu_{v}$, for $j=i+1, i+2, \ldots, m$. Observe that, if $\mu_{v}$ does not exist, $e_{i+1}=\left(z^{\prime}, v\right)$ and $m=i+1$. By the maximality of $G$ and by the fact that $G$ contains internal vertices, every vertex of $G$ has degree at least three. Hence, $m \geq 3$ and there exists a face delimited by a 3 -cycle containing edges $e_{1}$ and $e_{m}$. However, the third edge $e^{*}$ of such a cycle is an edge between a vertex in $\mu_{u}$ and a vertex in $\mu_{v}$ (if $\mu_{v}$ exists) or is an edge between a vertex in $\mu_{u}$ and $v$ (if $\mu_{v}$ does not exist), contradicting the fact that $\left(u^{\prime}, v^{\prime}\right)$ is the last edge in $E^{\prime}$. This completes the proof of the claim that $\mu_{z}$ exists.

Next, we claim that there exists an edge incident to $z^{\prime}$ and to a vertex belonging to $\mu_{z}$. The proof of such a claim can be done analogously to the proof that $\mu_{z}$ exists, namely by supposing, for a contradiction, that no edge incident to $z^{\prime}$ is incident to a vertex belonging to $\mu_{z}$, and by showing that this implies that the considered embedding is not $c$-planar (if a cluster containing both $u$ and $v$ and not containing $z$ exists), or that $\left(u^{\prime}, v^{\prime}\right)$ is not the last edge in $E^{\prime}$ (if a cluster containing both $u$ and $v$ and not containing $z$ does not exist).

Then, there exists an edge $\left(z^{\prime}, z_{2}\right)$ incident to $z^{\prime}$ such that $z_{2}$ belongs to $\mu_{z}$. Let $\left(z_{2}, z_{3}, \ldots, z_{Z}\right)$ be a chordless path such that $z_{Z}=z$ and $z_{i+1}$ is a descendant of $z_{i}$, for $2 \leq i \leq Z-1$. Such a path exists by Corollary 1 (notice that $\sigma\left(z_{2}\right)$ contains $z$ and contains neither $u$ nor $v)$. Consider path $\left(z_{1}, z_{2}, z_{3}, \ldots, z_{Z}\right)$. For each chord $\left(z_{1}, z_{j}\right)$, with $j>2$, replace the subpath of the current path by edge $\left(z_{1}, z_{j}\right)$. This results in a chordless path $\mathcal{P}_{z}$. By construction, paths $\mathcal{P}_{u}, \mathcal{P}_{v}$, and $\mathcal{P}_{z}$ satisfy Condition 1 of the lemma.

- Suppose that $\sigma\left(z^{\prime}\right)$ contains $u$ and $v$ and does not contain $z$. In the following we show how to find a path $\mathcal{P}_{z}^{1}=\left(z_{1}, z_{2}, \ldots, z_{Z^{*}}\right)$ such that: (i) $z_{1}=z^{\prime}$, (ii) $\sigma\left(z_{i}\right)$ contains $u$ and $v$ and does not contain $z$, for $1 \leq i \leq Z^{*}-2$, (iii) $\sigma\left(z Z^{*-1}\right)$ is 
either $\sigma(u, v, z)$ or a cluster containing $u$ and $v$ and not containing $z$, (iv) $z Z^{*}$ belongs to $\mu_{z}$, and (v) $\sigma\left(z_{i+1}\right)$ is an ancestor of $\sigma\left(z_{i}\right)$, for each $1 \leq i \leq Z^{*}-2$.

Assume that $\mathcal{P}_{z}^{1}$ has already been determined till a vertex $z_{i}$. We claim that there exists an edge incident to $z_{i}$ and to a vertex $z_{i+1}$ such that either $\sigma\left(z_{i+1}\right)$ is an ancestor of $\sigma\left(z_{i}\right)$ containing $u$ and $v$ and not containing $z$, or $\sigma\left(z_{i+1}\right)=\sigma(u, v, z)$, or $\sigma\left(z_{i+1}\right)$ contains $z$ and does not contain $u$ and $v$.

Suppose, for a contradiction, that every edge incident to $z_{i}$ is incident to a vertex belonging to $\mu_{u}$, or to a vertex belonging to $\mu_{v}$, or to a vertex belonging to a cluster that contains $u$ and $v$, that does not contain $z$, and that is a descendant of $\sigma\left(z_{i}\right)$. Observe that vertex $z_{i}$ is not adjacent to any vertex $v_{j}$ such that $\sigma\left(v_{j}\right)=\sigma\left(z_{i}\right)$, by Property $\mathrm{O} 3$ of Definition 1.

First, consider the case in which $\sigma\left(z_{i}\right) \neq \sigma(u, v)$, that is, there exists a child $\mu\left(u, v, \neg z_{i}\right)$ of $\sigma\left(z_{i}\right)$ in $T$ containing $u$ and $v$ and not containing $z$. Then, each edge incident to $z_{i}$ is also incident to a vertex belonging to $\mu\left(u, v, \neg z_{i}\right)$. Analogously as above, a contradiction can be reached by proving that there exists an edge $e^{*}$ that crosses twice the boundary $B\left(\mu\left(u, v, \neg z_{i}\right)\right)$ of $\mu\left(u, v, \neg z_{i}\right)$.

Second, consider the case in which $\sigma\left(z_{i}\right)=\sigma(u, v)$. Then, a contradiction can be reached by proving that: (i) if all the edges incident to $z_{i}$ are also incident to vertices belonging to $\mu_{u}$ (or if all the edges incident to $z_{i}$ are also incident to vertices belonging to $\mu_{v}$ ), then there exists an edge that crosses twice the boundary $B\left(\mu_{u}\right)$ of $\mu_{u}$ (resp. the boundary $B\left(\mu_{v}\right)$ of $\mu_{v}$ ); (ii) if some edges incident to $z_{i}$ are also incident to vertices in $\mu_{u}$ and some edges incident to $z_{i}$ are also incident to vertices in $\mu_{v}$, then $\left(u^{\prime}, v^{\prime}\right)$ is not the last edge in $E^{\prime}$.

This proves the claim, namely that there exists an edge incident to $z_{i}$ and to a vertex $z_{i+1}$ such that either $\sigma\left(z_{i+1}\right)$ is an ancestor of $\sigma\left(z_{i}\right)$ containing $u$ and $v$ and not containing $z$, or $\sigma\left(z_{i+1}\right)=\sigma(u, v, z)$, or $\sigma\left(z_{i+1}\right)$ contains $z$ and does not contain $u$ and $v$. The repetition of such an argument eventually leads to the choice of a vertex $z Z^{*}$ that belongs to $\mu_{z}$ or to the choice of a vertex $z Z^{*-1}$ such that $\sigma\left(z_{Z^{*}-1}\right)=\sigma(u, v, z)$. If $z_{Z^{*}}$ belongs to $\mu_{z}$, then $\mathcal{P}_{z}^{1}$ has already been determined satisfying the desired properties. If $\sigma\left(z_{Z^{*}-1}\right)=\sigma(u, v, z)$ then the same argument as above shows that there exists an edge $\left(z_{Z^{*}-1}, z_{Z^{*}}\right)$ such that $z_{Z^{*}}$ belongs to $\mu_{z}$, thus obtaining a path $\mathcal{P}_{z}^{1}$ satisfying the desired properties.

Let $\mathcal{P}_{z}^{2}=\left(z_{Z^{*}}, z_{Z^{*}+1}, \ldots, z_{Z}\right)$ be a chordless path such that $z_{Z}=z$, and $z_{i+1}$ is a descendant of $z_{i}$, for $Z^{*} \leq i \leq Z-1$. Such a path exists by Corollary 1 (notice that $\sigma\left(z Z^{*}\right)$ contains $z$ and contains neither $u$ nor $v$ ).

Finally, consider the path obtained by concatenating $\mathcal{P}_{z}^{1}$ and $\mathcal{P}_{z}^{2}$. Such a path may eventually have chords. For each chord $\left(z_{i}, z_{j}\right)$, with $j>i+1$, replace the subpath of the current path by edge $\left(z_{i}, z_{j}\right)$. This results in a chordless path $\mathcal{P}_{z}$. By construction, paths $\mathcal{P}_{u}, \mathcal{P}_{v}$, and $\mathcal{P}_{z}$ satisfy Condition 1 of the lemma.

A pseudo-code description of the algorithm for finding three paths satisfying Condition 1 of Lemma 12 or two paths satisfying Condition 2 of Lemma 12 is presented in Algorithm 5.

As in Lemma 12, let $C(G, T)$ be a maximal outerclustered graph, let $u, v$, and $z$ be the vertices incident to $o(G)$, and suppose that: (i) $\sigma(u) \neq \sigma(v), \sigma(u) \neq \sigma(z)$, and 


\section{Algorithm 5 TwO OR THREE PATHS IN MAXIMAL OUTERCLUSTERED GRAPHS}

Input: $C(G, T)$, as in Lemma 12.

Output: Paths $\mathcal{P}_{u}, \mathcal{P}_{v}$, and $\mathcal{P}_{z}$ satisfying Condition 1 of Lemma 12, or paths $\mathcal{P}_{u}$ and $\mathcal{P}_{v}$ satisfying Condition 2 of Lemma 12

1: $\mu_{u} \leftarrow$ biggest cluster containing $u$ and not containing $v$;

2: $\mu_{v} \leftarrow$ biggest cluster containing $v$ and not containing $u$ (if it exists);

3: if $\mu_{v}$ exists then

4: $\quad E^{\prime} \leftarrow$ set of edges whose end-vertices are one in $\mu_{u}$ and one in $\mu_{v}$;

5: else

6: $\quad E^{\prime} \leftarrow$ set of edges incident to $v$ whose other end-vertex is in $\mu_{u}$;

7: end if

8: $\left(u^{\prime}, v^{\prime}\right) \leftarrow$ last of the edges in $E^{\prime}$ incident to $l\left(\mu_{u}\right)$ starting from $(u, v)$;

9: $z^{\prime} \leftarrow$ vertex forming a face with $\left(u^{\prime}, v^{\prime}\right)$;

10: $\mathcal{P}_{u} \leftarrow$ edge $\left(z^{\prime}, u^{\prime}\right)$ plus the path obtained by Algorithm 3 on $C$, $u$, and $u^{\prime}$;

11: $\mathcal{P}_{v} \leftarrow$ edge $\left(z^{\prime}, v^{\prime}\right)$ plus the path obtained by Algorithm 3 on $C$, $v$, and $v^{\prime}$;

12: if $z^{\prime}=z$ then

13: $\quad$ return $\mathcal{P}_{u}$ and $\mathcal{P}_{v}$;

14: else

15: $\quad$ if $\sigma\left(z^{\prime}\right)$ contains $z$ and contains neither $u$ nor $v$ then

16: $\quad \mathcal{P}_{z} \leftarrow$ the path obtained by Algorithm 3 on $C, z$, and $z^{\prime}$;

17: $\quad$ return $\mathcal{P}_{u}, \mathcal{P}_{v}$, and $\mathcal{P}_{z}$;

18: $\quad$ end if

19: $\quad$ if $\sigma\left(z^{\prime}\right)=\sigma(u, v, z)$ then

20: $\quad\left(z^{\prime}, z_{2}\right) \leftarrow$ edge such that $z_{2}$ is in a cluster containing $z$ and neither $u$ nor $v$;

21: $\quad\left(z_{2}, z_{3}, \ldots, z_{Z}\right) \leftarrow$ path obtained by Algorithm 3 on $C, z$, and $z_{2}$;

22: $\quad \mathcal{P}_{z} \leftarrow$ the path obtained by Algorithm 4 on $G$ and $\left(z^{\prime}, z_{2}, z_{3}, \ldots, z_{Z}\right)$;

23: $\quad$ return $\mathcal{P}_{u}, \mathcal{P}_{v}$, and $\mathcal{P}_{z}$;

24: $\quad$ end if

25: $\quad$ if $\sigma\left(z^{\prime}\right)$ contains $u$ and $v$ and does not contain $z$ then

26: $\quad z_{j} \leftarrow z^{\prime}$

27: $\quad$ while $\sigma\left(z_{j}\right)$ contains $u$ and $v$ and does not contain $z$ do

28: $\quad z_{i} \leftarrow z_{j} ;\left(z_{i}, z_{i+1}\right) \leftarrow$ edge such that $\sigma\left(z_{i+1}\right)$ is not a descendant of $\sigma\left(z_{i}\right)$

29: $\quad$ add $\left(z_{i}, z_{i+1}\right)$ to the current path; $z_{j} \leftarrow z_{i+1}$;

30: $\quad$ end while

31: $\quad$ if $\sigma\left(z_{i}\right)=\sigma(u, v, z)$ then

32:

33:

34:

35:

36:

$\left(z_{i}, z_{i+1}\right) \leftarrow$ edge such that $\sigma\left(z_{i+1}\right)$ contains $z$ and neither $u$ nor $v$;

add $\left(z_{i}, z_{i+1}\right)$ to the current path;

end if

$\mathcal{P}_{z}^{1}=\left(z_{1}, z_{2}, \ldots, z_{Z^{*}}\right) \leftarrow$ the current path;

$\mathcal{P}_{z}^{2}=\left(z_{Z^{*}}, z_{Z^{*}+1}, \ldots, z_{Z}\right) \leftarrow$ path obtained by Algorithm 3 on $C, z$, and $z Z^{*}$

37: $\quad \mathcal{P}_{z} \leftarrow$ the path obtained by Algorithm 4 on $G$ and $\mathcal{P}_{z}^{1}$ plus $\mathcal{P}_{z}^{2}$;

38: $\quad$ return $\mathcal{P}_{u}, \mathcal{P}_{v}$, and $\mathcal{P}_{z}$;

39:

40: end if

\section{end if}




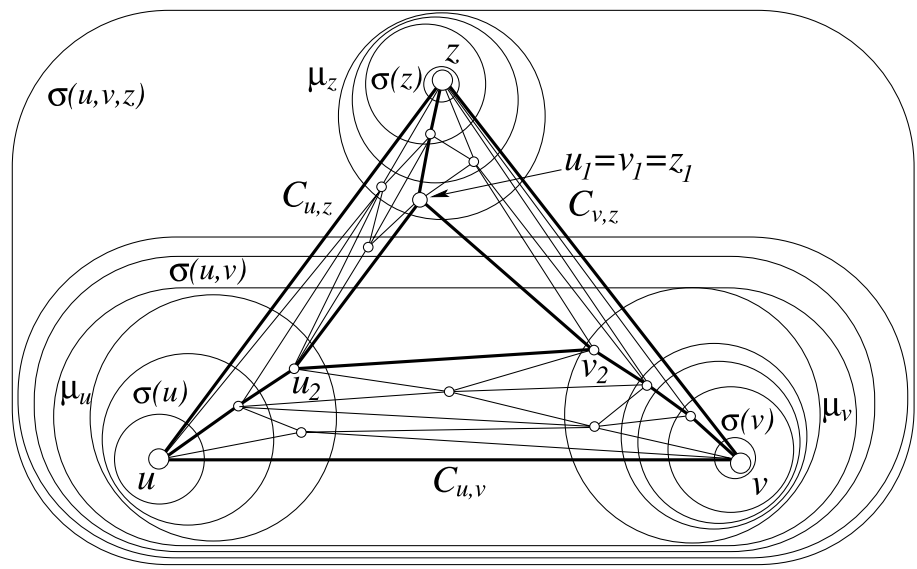

Fig. 24 Graphs $C_{u, v}, C_{u, z}$, and $C_{v, z}$ when Condition 1 of Lemma 12 holds. Thick edges show paths $\mathcal{P}_{u}$, $\mathcal{P}_{v}, \mathcal{P}_{z}$, and edges $(u, v),(u, z),(v, z)$, and $\left(u_{2}, v_{2}\right)$

$\sigma(v) \neq \sigma(z)$; (ii) if there exists a cluster containing exactly two vertices incident to $o(G)$, then such vertices are $u$ and $v$; (iii) if the smallest containing cluster of one of $u$ and $v$ contains the other one, then $\sigma(v)$ contains $u$; and (iv) $G$ has internal vertices.

Suppose that Condition 1 of Lemma 12 holds (see Fig. 24). Denote by $C_{u, v}$, by $C_{u, z}$, and by $C_{v, z}$ the clustered graphs whose underlying graphs $G_{u, v}, G_{u, z}$, and $G_{v, z}$ are the subgraphs of $G$ induced by the vertices incident to and internal to cycle $\mathcal{C}_{u, v} \equiv(u, v) \cup\left(\mathcal{P}_{u} \backslash\left\{u_{1}\right\}\right) \cup\left(u_{2}, v_{2}\right) \cup\left(\mathcal{P}_{v} \backslash\left\{v_{1}\right\}\right)$, incident to and internal to cycle $\mathcal{C}_{u, z} \equiv(u, z) \cup \mathcal{P}_{u} \cup \mathcal{P}_{z}$, and incident to and internal to cycle $\mathcal{C}_{v, z} \equiv(v, z) \cup \mathcal{P}_{v} \cup \mathcal{P}_{z}$, and whose inclusion trees $T_{u, v}, T_{u, z}$, and $T_{v, z}$ are the subtrees of $T$ induced by the clusters containing vertices of $G_{u, v}$, of $G_{u, z}$, and of $G_{v, z}$, respectively.

Lemma $13 C_{u, v}, C_{u, z}$, and $C_{v, z}$ are linearly-ordered outerclustered graphs.

Proof We prove the statement for $C_{u, v}$, the proofs for $C_{u, z}$ and $C_{v, z}$ being analogous. Refer to Fig. 25. The outer face of $G_{u, v}$ is delimited by a simple cycle $\mathcal{C}_{u, v}$. We prove that the boundary of each cluster $\mu$ containing vertices of $\mathcal{C}_{u, v}$ and not containing all the vertices of $\mathcal{C}_{u, v}$ intersects $\mathcal{C}_{u, v}$ exactly twice. By construction, each cluster containing both $u$ and $v$ contains all the vertices of $G_{u, v}$ and each cluster containing neither $u$ nor $v$ does not contain any vertex of $G_{u, v}$. Finally, each cluster $\mu$ containing $u$ and not containing $v$ (the arguments for each cluster $\mu$ containing $v$ and not containing $u$ being analogous) intersects edge $(u, v)$ exactly once, intersects path $\left(\mathcal{P}_{u} \backslash\left\{u_{1}\right\}\right) \cup\left(u_{2}, v_{2}\right)$ exactly once, and does not intersect path $\mathcal{P}_{v} \backslash\left\{v_{1}\right\}$. It follows that $\mu$ intersects $\mathcal{C}_{u, v}$ twice. By Lemma $1, C_{u, v}$ is a biconnected internallytriangulated outerclustered graph.

Consider the sequence of clusters $\Sigma=\mu_{1}, \mu_{2}, \ldots, \mu_{k}$ such that: (i) $\mu_{1}=\sigma(u)$, (ii) $\mu_{i+1}$ is the parent of $\mu_{i}$ in $T_{u, v}$, for $i=1,2, \ldots, h-2$, (iii) $\mu_{h-1}$ is the biggest cluster containing $u$ and not containing $v$, (iv) $\mu_{h}$ is $\sigma(u, v)$, (v) $\mu_{h+1}$ is the biggest cluster containing $v$ and not containing $u$, (vi) $\mu_{i+1}$ is the only child of $\mu_{i}$ in $T_{u, v}$ containing $v$, for $i=h+1, h+2, \ldots, k-1$, and (vii) $\mu_{k}=\sigma(v)$. Notice that, if 


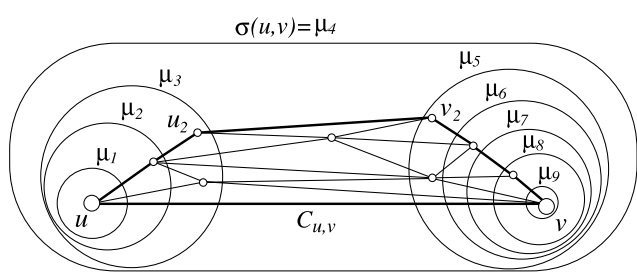

(a)

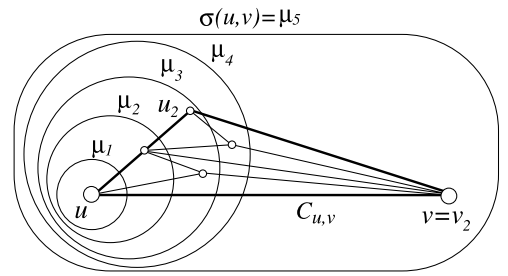

(b)

Fig. 25 A closer look to $C_{u, v}$. Cluster sequence $\Sigma$ is shown. (a) A cluster containing $v$ and not containing $u$ exists. (b) A cluster containing $v$ and not containing $u$ does not exist

a cluster containing $v$ and not containing $u$ does not exist, then $\mu_{h}=\mu_{k}$. In the following we prove that $C_{u, v}$ is linearly-ordered according to $\Sigma$.

We prove that $C_{u, v}$ satisfies Property LO1 of Definition 2. Each vertex $x$ in $G_{u, v}$ either belongs to $\mu_{h-1}$, or to $\mu_{h+1}$, or is such that $\sigma(x)=\sigma(u, v)$. Namely, if $\mu_{v}$ exists, then all the vertices incident to $o\left(G_{u, v}\right)$ either belong to $\mu_{h-1}$ or to $\mu_{h+1}$, by construction; hence, all the vertices of $G_{u, v}$ belong to the smallest cluster containing both $\mu_{h-1}$ and $\mu_{h+1}$, that is $\sigma(u, v)$, and do not belong to any cluster containing neither $u$ nor $v$. If $\mu_{v}$ does not exist, then all the vertices incident to $o\left(G_{u, v}\right)$, except for $v$, belong to $\mu_{h-1}$ and $v$ belongs to $\sigma(u, v)$; hence, all the vertices of $G_{u, v}$ belong to the smallest cluster containing both $\mu_{h-1}$ and $v$, that is $\sigma(u, v)$, and do not belong to any cluster containing neither $u$ nor $v$. Further, $\Sigma$ includes all and only the clusters containing at least one out of $u$ and $v$, with the exception of the clusters containing both $u$ and $v$ and ancestors of $\sigma(u, v)$; however, each cluster containing both $u$ and $v$, and ancestor of $\sigma(u, v)$ is different from $\sigma(x)$, for any vertex $x$ of $G_{u, v}$, since $x$ is also contained in $\sigma(u, v)$. Since, for each internal vertex $x$ of $G, \sigma(x)$ is a cluster containing at least one out of $u$ and $v$, then $\sigma(x)=\mu_{i}$, for some $1 \leq i \leq k$.

We prove that $C_{u, v}$ satisfies Property LO2. Edge $(u, v)$ and the path obtained by concatenating $\mathcal{P}_{u} \backslash\left\{u_{1}\right\},\left(u_{2}, v_{2}\right)$, and $\mathcal{P}_{v} \backslash\left\{v_{1}\right\}$ are monotone paths delimiting $o\left(G_{u, v}\right)$.

We prove that $C_{u, v}$ satisfies Property LO3. By construction, $\mu_{i+1}$ is the parent of $\mu_{i}$, for $i=1,2, \ldots, h-2$, and $\mu_{i+1}$ is a child of $\mu_{i}$, for $i=h+1, h+2, \ldots, k-1$. Hence, in order to prove that $C_{u, v}$ satisfies Property LO3, it suffices to show that $\mu_{h}$ is the parent of $\mu_{h-1}$ and that $\mu_{h}$ is the parent of $\mu_{h+1}$. By construction, $\mu_{h-1}$ (resp. $\mu_{h+1}$ ) is the biggest cluster containing $u$ and not containing $v$ (resp. containing $v$ and not containing $u$ ). Hence, the parent of $\mu_{h-1}$ (resp. of $\mu_{h+1}$ ) is $\sigma(u, v)$, that by definition is $\mu_{h}$.

Suppose that Condition 2 of Lemma 12 holds (see Fig. 26).

Denote by $C_{u, v}$, by $C_{u, z}$, and by $C_{v, z}$ the clustered graphs whose underlying graphs $G_{u, v}, G_{u, z}$, and $G_{v, z}$ are the subgraphs of $G$ induced by the vertices incident to and internal to cycle $\mathcal{C}_{u, v} \equiv(u, v) \cup\left(\mathcal{P}_{u} \backslash\left\{u_{1}\right\}\right) \cup\left(u_{2}, v_{2}\right) \cup\left(\mathcal{P}_{v} \backslash\left\{v_{1}\right\}\right)$, incident to and internal to cycle $\mathcal{C}_{u, z} \equiv(u, z) \cup \mathcal{P}_{u}$, and incident to and internal to cycle $\mathcal{C}_{v, z} \equiv(v, z) \cup \mathcal{P}_{v}$, and whose inclusion trees $T_{u, v}, T_{u, z}$, and $T_{v, z}$ are the subtrees of $T$ induced by the clusters containing vertices of $G_{u, v}$, of $G_{u, z}$, and of $G_{v, z}$, 


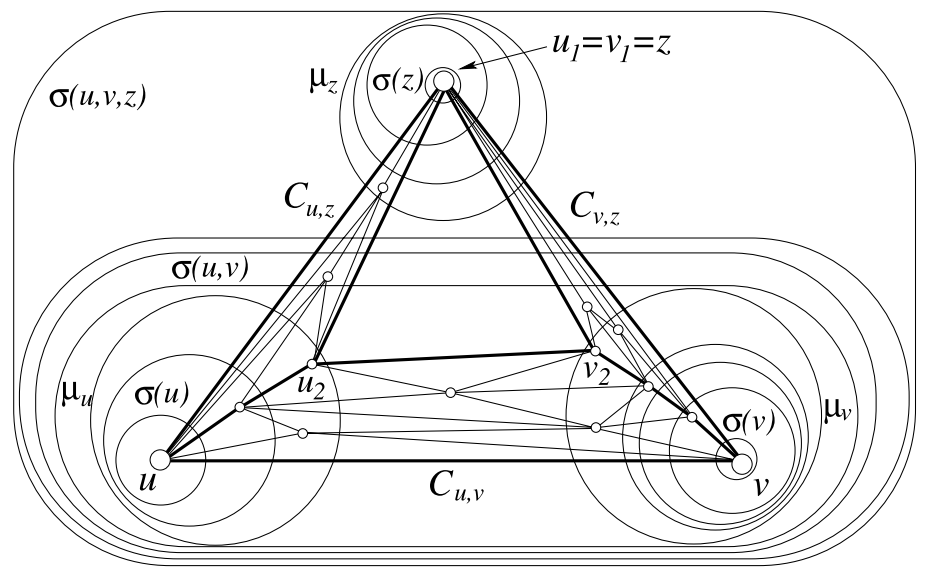

Fig. 26 Graphs $C_{u, v}, C_{u, z}$, and $C_{v, z}$ when Condition 2 of Lemma 12 holds. Thick edges show paths $\mathcal{P}_{u}$, $\mathcal{P}_{v}$, and edges $(u, v),(u, z),(v, z)$, and $\left(u_{2}, v_{2}\right)$

respectively. We have the following lemma, whose proof is analogous to the one of Lemma 13.

Lemma $14 C_{u, v}, C_{u, z}$, and $C_{v, z}$ are linearly-ordered outerclustered graphs.

We are now ready to exhibit the main theorem of this section.

Theorem 2 Let $C(G, T)$ be a maximal outerclustered graph. Then, for every triangular-convex-separated drawing $\Gamma\left(C_{o}\right)$ of $C_{o}$, there exists an internally-convexseparated drawing $\Gamma(C)$ of $C$ completing $\Gamma\left(C_{o}\right)$.

Proof Let $u, v$, and $z$ be the vertices incident to $o(G)$. Suppose that $G$ has internal vertices, otherwise $C_{o}$ and $C$ are the same graph, and the statement trivially follows.

If $\sigma(u)=\sigma(v)$, then we claim that $C$ is a linearly-ordered outerclustered graph. Refer to Fig. 27. Observe that $C$ is a maximal outerclustered graph by hypothesis, hence $G$ is biconnected and internally-triangulated.

Define the sequence of clusters $\Sigma=\mu_{1}, \mu_{2}, \ldots, \mu_{k}$ such that: (i) $\mu_{1}=\sigma(u)=$ $\sigma(v)$, (ii) $\mu_{i+1}$ is the parent of $\mu_{i}$ in $T$, for $i=1,2, \ldots, h-2$, (iii) $\mu_{h-1}$ is the biggest cluster containing $u$ and $v$ and not containing $z$, if any such a cluster exists, (iv) $\mu_{h}=\sigma(u, v, z)$, (v) $\mu_{h+1}$ is the biggest cluster containing $z$ and not containing $u$ and $v$, if any such a cluster exists, (vi) $\mu_{i+1}$ is the only child of $\mu_{i}$ in $T$ containing $z$, for $i=h+1, h+2, \ldots, k-1$, and (vii) $\mu_{k}=\sigma(z)$. We claim that $C$ is linearlyordered according to $\Sigma$.

We prove that $C$ satisfies Property LO1 of Definition 2. By construction, $\Sigma$ includes all the clusters containing at least one out of $u, v$, and $z$, with the exception of the clusters that contain all of $u, v$, and $z$ and that are ancestors of $\sigma(u, v, z)$; however, each cluster containing all of $u, v$, and $z$ and ancestor of $\sigma(u, v, z)$ is different from $\sigma(x)$, for any vertex $x$ of $G$, since $x$ is also contained in $\sigma(u, v, z)$. Since, for 


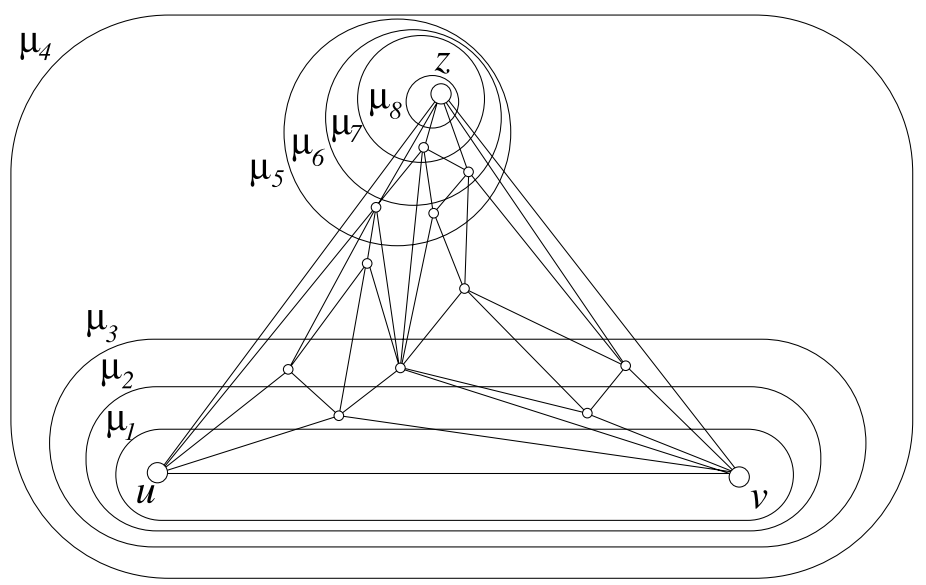

Fig. 27 If $\sigma(u)=\sigma(v)$, then $C$ is a linearly-ordered outerclustered graph

each vertex $x$ of $G, \sigma(x)$ is a cluster containing at least one out of $u, v$, and $z$, by definition of outerclustered graph, then $\sigma(x)=\mu_{i}$, for some $1 \leq i \leq k$.

We prove that $C$ satisfies Property LO2. Path $(u, v, z)$ and edge $(u, z)$ are monotone paths delimiting $o(G)$.

We prove that $C$ satisfies Property LO3. By construction, $\mu_{i+1}$ is the parent of $\mu_{i}$, for $i=1,2, \ldots, h-2$, and $\mu_{i+1}$ is a child of $\mu_{i}$, for $i=h+1, h+2, \ldots, k-1$. Hence, in order to prove that $C$ satisfies Property LO3, it suffices to show that $\mu_{h}$ is the parent of $\mu_{h-1}$ and that $\mu_{h}$ is the parent of $\mu_{h+1}$. By construction, $\mu_{h-1}$ (resp. $\mu_{h+1}$ ) is the biggest cluster containing $u$ and $v$ and not containing $z$ (resp. containing $z$ and not containing $u$ and $v$ ). Hence, the parent of $\mu_{h-1}$ (resp. of $\left.\mu_{h+1}\right)$ is $\sigma(u, v, z)$, that by definition is $\mu_{h}$.

Analogously, if $\sigma(u)=\sigma(z)$ or if $\sigma(v)=\sigma(z), C$ is a linearly-ordered outerclustered graph. By Lemma 4, a triangular-convex-separated drawing of $C_{o}$ is also a convex-separated drawing of $C_{o}$, hence in such cases the theorem directly follows from Theorem 1.

Now suppose that $\sigma(u) \neq \sigma(v), \sigma(u) \neq \sigma(z)$, and $\sigma(v) \neq \sigma(z)$. Suppose also that, if there exists a cluster containing exactly two vertices incident to $o(G)$, then such vertices are $u$ and $v$. The cases in which such vertices are $u$ and $z$, or $v$ and $z$ can be treated analogously. Suppose finally that if the smallest containing cluster of one of $u$ and $v$ contains the other one, then $\sigma(v)$ contains $u$. The case in which $\sigma(u)$ contains $v$ can be treated analogously.

By Lemma 12, there exist three paths $\mathcal{P}_{u}=\left(u_{1}, u_{2}, \ldots, u_{U}\right), \mathcal{P}_{v}=\left(v_{1}, v_{2}\right.$, $\left.\ldots, v_{V}\right)$, and $\mathcal{P}_{z}=\left(z_{1}, z_{2}, \ldots, z_{Z}\right)$ satisfying Condition 1 of Lemma 12, or there exist two paths $\mathcal{P}_{u}=\left(u_{1}, u_{2}, \ldots, u_{U}\right)$ and $\mathcal{P}_{v}=\left(v_{1}, v_{2}, \ldots, v_{V}\right)$ satisfying Condition 2 of Lemma 12.

Suppose that Condition 1 of Lemma 12 holds.

By Lemma 13, $C_{u, v}\left(G_{u, v}, T_{u, v}\right), C_{u, z}\left(G_{u, z}, T_{u, z}\right)$, and $C_{v, z}\left(G_{v, z}, T_{v, z}\right)$ are linearly-ordered outerclustered graphs. 


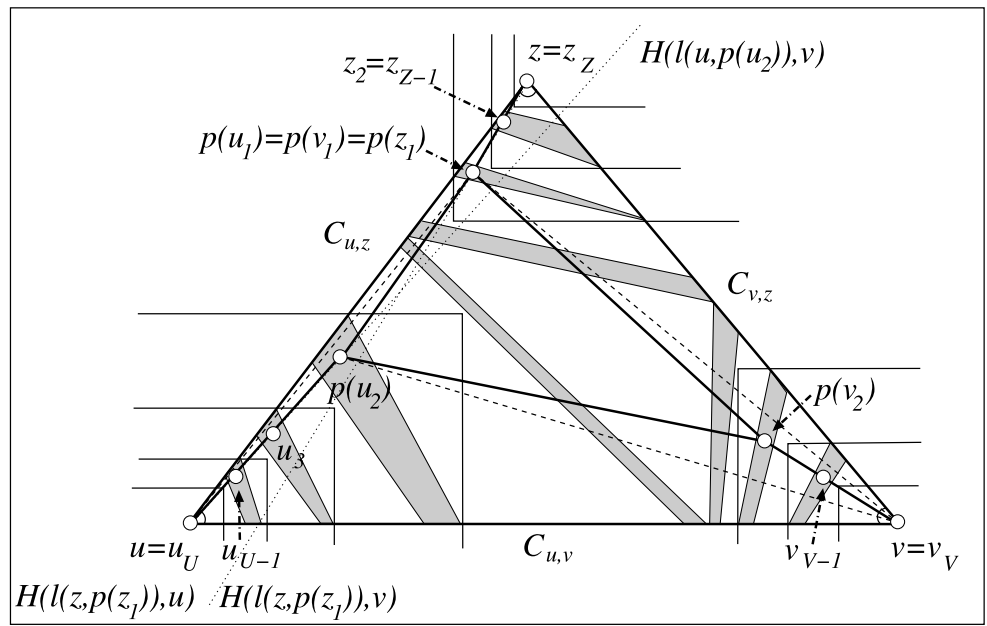

Fig. 28 Construction of drawings $\Gamma\left(\mathcal{C}_{u, v}, T_{u, v}\right), \Gamma\left(\mathcal{C}_{u, z}, T_{u, z}\right)$, and $\Gamma\left(\mathcal{C}_{v, z}, T_{v, z}\right)$ when Condition 1 of Lemma 12 holds

Refer to Fig. 28. Suppose that $U>2$ and that $V>2$. Consider a child $\sigma^{\prime}\left(z_{1}\right)$ of $\sigma\left(z_{1}\right)$ such that: (i) if $\sigma\left(z_{1}\right)$ contains $z$ and does not contain $u$ and $v$, then $\sigma^{\prime}\left(z_{1}\right)$ is the unique child of $\sigma\left(z_{1}\right)$; (ii) if $\sigma\left(z_{1}\right)=\sigma(u, v, z)$, then $\sigma^{\prime}\left(z_{1}\right)$ is the unique child of $\sigma\left(z_{1}\right)$ containing $z$; (iii) if $\sigma\left(z_{1}\right)$ contains $u$ and $v$ and does not contain $z$, then $\sigma^{\prime}\left(z_{1}\right)$ is the unique child of $\sigma\left(z_{1}\right)$ containing $u$. The existence of such clusters in each of the three cases can be proved as in the proof of Lemma 12. Notice that, in any case, one of the two polygonal lines obtained as intersection of the triangle representing $(u, v, z)$ and $R\left(\sigma\left(z_{1}\right), \sigma^{\prime}\left(z_{1}\right)\right)$ lies on edge $(u, z)$. Consider a point $p\left(z_{1}\right)$ of $\operatorname{int}\left(R\left(\sigma\left(z_{1}\right), \sigma^{\prime}\left(z_{1}\right)\right)\right)$ arbitrarily close to edge $(u, z)$. Place $u_{1}=v_{1}=z_{1}$ at $p\left(z_{1}\right)$.

Let $\sigma^{\prime}\left(z_{i}\right)$ be any child of $\sigma\left(z_{i}\right)$ such that the boundary of $\sigma^{\prime}\left(z_{i}\right)$ has intersection with $(u, z)$, for each $2 \leq i \leq Z-1$. Notice that such a child is unique, except for the case in which $\sigma\left(z_{i}\right)=\sigma(u, v, z)$, in which $\sigma\left(z_{i}\right)$ may have two children satisfying the required properties, namely the biggest cluster containing $u$ and not containing $z$ and the biggest cluster containing $z$ and not containing $u$. Draw a straight-line segment $\overline{p\left(z_{1}\right) z}$ and place $z_{i}$ at any point of the segment $\operatorname{int}\left(R\left(\sigma\left(z_{i}\right), \sigma^{\prime}\left(z_{i}\right)\right)\right) \cap \overline{p\left(z_{1}\right) z}$, for each $2 \leq l \leq Z-1$.

Denote by $T\left(u, v, p\left(z_{1}\right)\right)$ the triangle having $u, v$, and $p\left(z_{1}\right)$ as vertices. Denote also by $H\left(l\left(z, p\left(z_{1}\right)\right), u\right)$ (by $\left.H\left(l\left(z, p\left(z_{1}\right)\right), v\right)\right)$ the open half-plane delimited by the line through $z$ and $p\left(z_{1}\right)$, and containing $u$ (resp. containing $v$ ). Consider the unique child $\sigma^{\prime}\left(u_{2}\right)$ of $\sigma\left(u_{2}\right)$. Notice that one of the two polygonal lines obtained as intersection of the triangle representing $(u, v, z)$ and $R\left(\sigma\left(u_{2}\right), \sigma^{\prime}\left(u_{2}\right)\right)$ lies on edge $(u, z)$. Consider a point $p\left(u_{2}\right)$ in $H\left(l\left(z, p\left(z_{1}\right)\right), u\right) \cap \operatorname{int}\left(T\left(u, v, p\left(z_{1}\right)\right)\right) \cap$ $\operatorname{int}\left(R\left(\sigma\left(u_{2}\right), \sigma^{\prime}\left(u_{2}\right)\right)\right)$ arbitrarily close to edge $\left(u, p\left(z_{1}\right)\right)$. Place $u_{2}$ at $p\left(u_{2}\right)$.

Let $\sigma^{\prime}\left(u_{i}\right)$ be the unique child of $\sigma\left(u_{i}\right)$, for each $3 \leq i \leq U-1$. Draw a straightline segment $\overline{p\left(u_{2}\right) u}$ and place $u_{i}$ at any point of the segment $\operatorname{int}\left(R\left(\sigma\left(u_{i}\right), \sigma^{\prime}\left(u_{i}\right)\right)\right) \cap$ $\overline{p\left(u_{2}\right) u}$, for each $3 \leq i \leq U-1$.

Denote by $T\left(p\left(u_{2}\right), v, p\left(z_{1}\right)\right)$ the triangle having $p\left(u_{2}\right), v$, and $p\left(z_{1}\right)$ as vertices. Denote also by $H\left(l\left(u, p\left(u_{2}\right)\right), v\right)$ the open half-plane delimited by the line through 
$u$ and $p\left(u_{2}\right)$, and containing $v$. Consider the unique child $\sigma^{\prime}\left(v_{2}\right)$ of $\sigma\left(v_{2}\right)$. Consider any point $p\left(v_{2}\right)$ in $H\left(l\left(z, p\left(z_{1}\right)\right), v\right) \cap H\left(l\left(u, p\left(u_{2}\right)\right), v\right) \cap \operatorname{int}\left(T\left(p\left(u_{2}\right), v, p\left(z_{1}\right)\right)\right) \cap$ $\operatorname{int}\left(R\left(\sigma\left(v_{2}\right), \sigma^{\prime}\left(v_{2}\right)\right)\right)$. Observe that, since $p\left(z_{1}\right)$ and $p\left(u_{2}\right)$ are arbitrarily close to edge $(u, z)$, both half-planes $H\left(l\left(z, p\left(z_{1}\right)\right), v\right)$ and $H\left(l\left(u, p\left(u_{2}\right)\right), v\right)$ entirely contain triangle $T(u, v, z)$, except for an arbitrarily small strip close to edge $(u, z)$. This guarantees that $H\left(l\left(z, p\left(z_{1}\right)\right), v\right) \cap H\left(l\left(u, p\left(u_{2}\right)\right), v\right) \cap \operatorname{int}\left(T\left(p\left(u_{2}\right), v, p\left(z_{1}\right)\right)\right) \cap$ $\operatorname{int}\left(R\left(\sigma\left(v_{2}\right), \sigma^{\prime}\left(v_{2}\right)\right)\right)$ is a convex non-empty region. Then, place $v_{2}$ at $p\left(v_{2}\right)$.

Let $\sigma^{\prime}\left(v_{i}\right)$ be the unique child of $\sigma\left(v_{i}\right)$, for each $3 \leq i \leq V-1$. Draw a straightline segment $\overline{p\left(v_{2}\right) v}$ and place $v_{i}$ at any point of the segment $\operatorname{int}\left(R\left(\sigma\left(v_{i}\right), \sigma^{\prime}\left(v_{i}\right)\right)\right) \cap$ $\overline{p\left(v_{2}\right) v}$, for each $3 \leq i \leq V-1$. Straightforward modifications make the described algorithm work also for the cases in which $U=2$ and/or $V=2$. Such modifications are described in Algorithm 7 below.

Denote by $\Gamma\left(\mathcal{C}_{u, v}, T_{u, v}\right)$, by $\Gamma\left(\mathcal{C}_{u, z}, T_{u, z}\right)$, and by $\Gamma\left(\mathcal{C}_{v, z}, T_{v, z}\right)$ the constructed drawings of $\left(\mathcal{C}_{u, v}, T_{u, v}\right)$, of $\left(\mathcal{C}_{u, z}, T_{u, z}\right)$, and of $\left(\mathcal{C}_{v, z}, T_{v, z}\right)$, respectively. We have the following:

Lemma $15 \Gamma\left(\mathcal{C}_{u, v}, T_{u, v}\right), \Gamma\left(\mathcal{C}_{u, z}, T_{u, z}\right)$, and $\Gamma\left(\mathcal{C}_{v, z}, T_{v, z}\right)$ are convex-separated drawings of the outer faces of $C_{u, v}\left(G_{u, v}, T_{u, v}\right), C_{u, z}\left(G_{u, z}, T_{u, z}\right)$, and $C_{v, z}\left(G_{v, z}\right.$, $\left.T_{v, z}\right)$, respectively.

Proof We prove the statement for $\Gamma\left(\mathcal{C}_{u, v}, T_{u, v}\right)$, the proof for $\Gamma\left(\mathcal{C}_{u, z}, T_{u, z}\right)$, and $\Gamma\left(\mathcal{C}_{v, z}, T_{v, z}\right)$ being analogous. Denote by $P, P_{u, v}, P_{u, z}$, and $P_{v, z}$ the polygons representing cycles $\mathcal{C}, \mathcal{C}_{u, v}, \mathcal{C}_{u, z}$, and $\mathcal{C}_{v, z}$, respectively. The drawing is straight-line and rectangular by construction. The absence of edge crossings easily descends from the construction. The absence of region-region crossings descends from the fact that no cluster is drawn by the algorithm.

We prove that $\Gamma\left(\mathcal{C}_{u, v}, T_{u, v}\right)$ has no edge-region crossings. Suppose, for a contradiction, that there is an edge-region crossing between an edge $e$ and a cluster $v$. If both end-vertices of $e$ belong to $v$ then, by the convexity of $v, e$ is internal to $v$; if one end-vertex of $e$ belongs to $v$ then, by the convexity of $v, e$ crosses $v$ exactly once; hence, it can be assumed that both the end-vertices of $e$ do not belong to $v$. Any cluster containing both $u$ and $v$ contains all the vertices of $G_{u, v}$, hence it contains all the drawing of $\mathcal{C}_{u, v}$ and does not cross $e$. Hence, it can be assumed that $v$ contains $u$ and does not contain $v$, or vice versa. Suppose that $v$ contains $u$ and does not contain $v$, the other case being analogous. Consider the parent $\mu$ of $v$ in $T_{u, v}$. Such a parent exists since otherwise $v$ would be the root of $T$, contradicting the fact that $v$ does not contain $v$. By definition of triangular-convex-separated drawing, there exists a convex region $R(\mu, v)$ with the properties described in Definition 4; one of the sides of such a region separates $v$ from the rest of the drawing, thus avoiding an edge-region crossing between $e$ and $v$. More precisely, since $C(G, T)$ is an outerclustered graph, $v$ has exactly two incident edges $e_{1}(v)$ and $e_{2}(v)$ incident to $o(G)$. Denote by $u\left(e_{1}(v)\right)$ and $u\left(e_{2}(v)\right)$ the end-vertices of $e_{1}(v)$ and $e_{2}(v)$ belonging to $v$. Denote by $p\left(l_{1}\right)$ the endpoint of $l_{1}(\mu, v)$ that lies on $e_{1}(v)$ (if both endpoints of $l_{1}(\mu, v)$ lie on $e_{1}(v)$, then $p\left(l_{1}\right)$ is the one that is closer to $\left.u\left(e_{1}(v)\right)\right)$. Note that an endpoint of $l_{1}(\mu, v)$ lying on $e_{1}(\nu)$ exists as $\Gamma$ satisfies Property CS3. Analogously define $p\left(l_{2}\right)$. Then, segment $\overline{p\left(l_{1}\right) p\left(l_{2}\right)}$ splits $P$ into two disjoint convex polygons $P^{\prime}$ and $P^{\prime \prime}$, where $P^{\prime}$ contains 


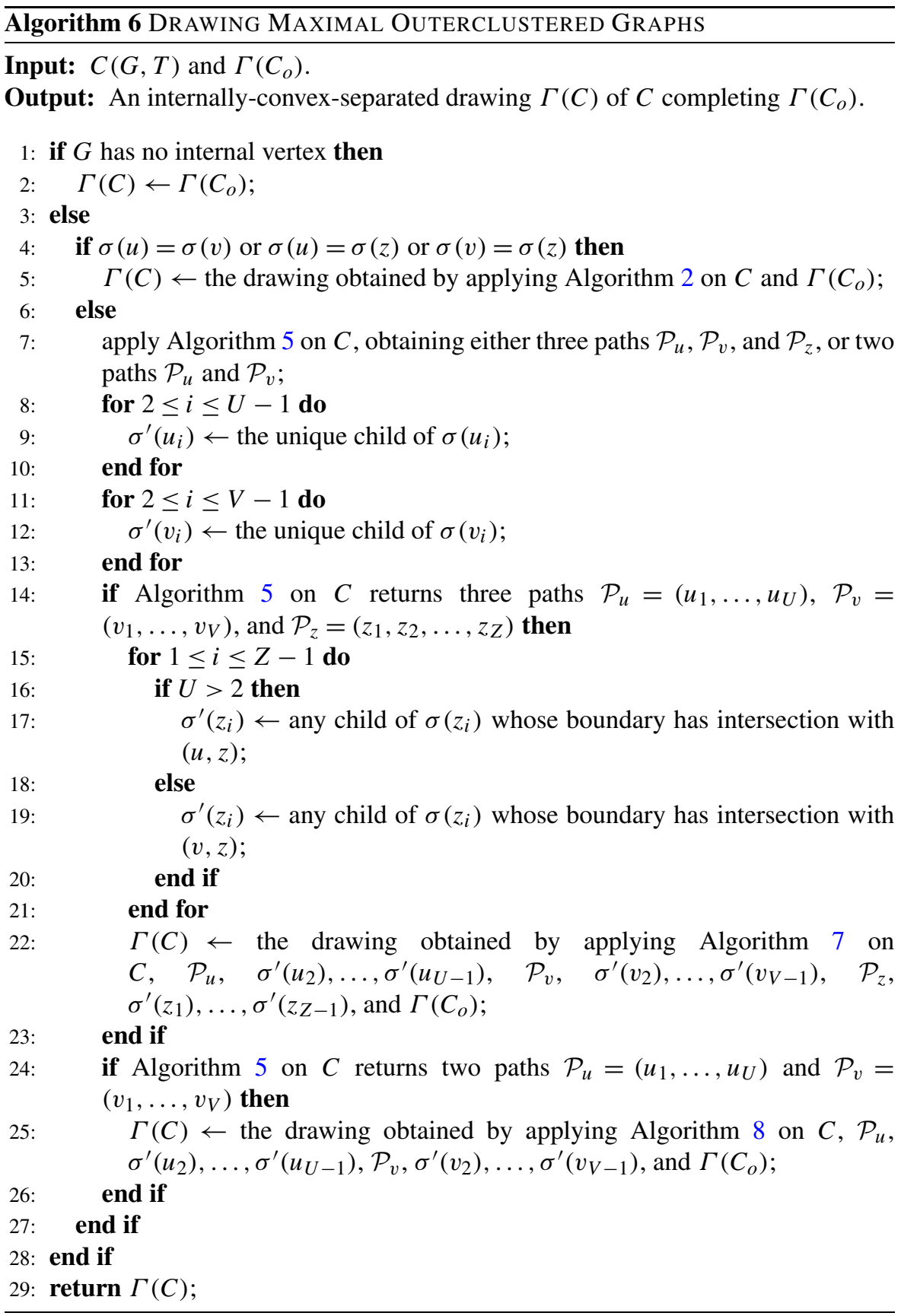

all and only the vertices belonging to $v$ and $P^{\prime \prime}$ contains all and only the vertices not belonging to $v$, as $\Gamma\left(C_{o}\right)$ satisfies Property CS3. By convexity, $e$ is internal to $P^{\prime \prime}$, while the part of $v$ inside $P$ is internal to $P^{\prime}$. Hence, $e$ does not cross $v$. 


\section{$\overline{\text { Algorithm } 7 \text { Drawing Maximal Outerclustered Graphs With Three }}$ PATHS \\ Input: $C, \mathcal{P}_{u}=\left(u_{1}, \ldots, u_{U}\right), \sigma^{\prime}\left(u_{2}\right), \ldots, \sigma^{\prime}\left(u_{U-1}\right), \mathcal{P}_{v}=\left(v_{1}, \ldots, v_{V}\right), \sigma^{\prime}\left(v_{2}\right)$, $\ldots, \sigma^{\prime}\left(v_{V-1}\right), \mathcal{P}_{z}=\left(z_{1}, z_{2}, \ldots, z_{Z}\right), \sigma^{\prime}\left(z_{1}\right), \ldots, \sigma^{\prime}\left(z_{Z-1}\right)$, and $\Gamma\left(C_{o}\right)$;}

Output: An internally-convex-separated drawing $\Gamma(C)$ of $C$ completing $\Gamma\left(C_{o}\right)$.

1: if $U>2$ then

2: $\quad p\left(z_{1}\right) \leftarrow$ point of $\operatorname{int}\left(R\left(\sigma\left(z_{1}\right), \sigma^{\prime}\left(z_{1}\right)\right)\right)$ close to $(u, z)$; place $z_{1}$ at $p\left(z_{1}\right)$;

3: $\quad H\left(l\left(z, p\left(z_{1}\right)\right), u\right), H\left(l\left(z, p\left(z_{1}\right)\right), v\right) \leftarrow$ the open half-planes delimited by the line through $z$ and $p\left(z_{1}\right)$, and containing $u$ and $v$, respectively;

4: $\quad p\left(u_{2}\right) \leftarrow$ point of $H\left(l\left(z, p\left(z_{1}\right)\right), u\right) \cap \operatorname{int}\left(T\left(u, v, p\left(z_{1}\right)\right)\right) \cap$ $\operatorname{int}\left(R\left(\sigma\left(u_{2}\right), \sigma^{\prime}\left(u_{2}\right)\right)\right)$ close to $\left(u, p\left(z_{1}\right)\right)$; place $u_{2}$ at $p\left(u_{2}\right)$;

5: $\quad$ apply Algorithm 9 on $\mathcal{P}_{u} \backslash\left\{u_{1}\right\}, R\left(\sigma\left(u_{3}\right), \sigma^{\prime}\left(u_{3}\right)\right), R\left(\sigma\left(u_{4}\right), \sigma^{\prime}\left(u_{4}\right)\right), \ldots$, $R\left(\sigma\left(u_{U-1}\right), \sigma^{\prime}\left(u_{U-1}\right)\right)$, and $\overline{p\left(u_{2}\right) u}$;

6: $\quad$ if $V>2$ then

7: $\quad H\left(l\left(u, p\left(u_{2}\right)\right), v\right) \leftarrow$ the open half-plane delimited by the line through $u$ and $p\left(u_{2}\right)$ and containing $v$;

8: $\quad p\left(v_{2}\right) \leftarrow$ point of $H\left(l\left(z, p\left(z_{1}\right)\right), v\right) \cap H\left(l\left(u, p\left(u_{2}\right)\right), v\right) \cap$ $\operatorname{int}\left(T\left(p\left(u_{2}\right), v, p\left(z_{1}\right)\right)\right) \cap \operatorname{int}\left(R\left(\sigma\left(v_{2}\right), \sigma^{\prime}\left(v_{2}\right)\right)\right)$; place $v_{2}$ at $p\left(v_{2}\right)$;

9: $\quad$ apply Algorithm 9 on $\mathcal{P}_{v} \backslash\left\{v_{1}\right\}, R\left(\sigma\left(v_{3}\right), \sigma^{\prime}\left(v_{3}\right)\right), R\left(\sigma\left(v_{4}\right), \sigma^{\prime}\left(v_{4}\right)\right), \ldots$, $R\left(\sigma\left(v_{V-1}\right), \sigma^{\prime}\left(v_{V-1}\right)\right)$, and $\overline{p\left(v_{2}\right) v}$;

10:

\section{end if}

11: else

12: $\quad$ if $V>2$ then

13: $\quad p\left(z_{1}\right) \leftarrow$ point of $\operatorname{int}\left(R\left(\sigma\left(z_{1}\right), \sigma^{\prime}\left(z_{1}\right)\right)\right)$ close to $(v, z)$; place $z_{1}$ at $p\left(z_{1}\right)$;

14: $\quad H\left(l\left(z, p\left(z_{1}\right)\right), v\right) \leftarrow$ the open half-plane delimited by the line through $z$ and $p\left(z_{1}\right)$, and containing $v$;

15: $\quad p\left(v_{2}\right) \leftarrow$ point of $H\left(l\left(z, p\left(z_{1}\right)\right), v\right) \cap \operatorname{int}\left(T\left(u, v, p\left(z_{1}\right)\right)\right) \cap$ $\operatorname{int}\left(R\left(\sigma\left(v_{2}\right), \sigma^{\prime}\left(v_{2}\right)\right)\right)$ close to $\left(v, p\left(z_{1}\right)\right)$; place $v_{2}$ at $p\left(v_{2}\right)$;

16: $\quad$ apply Algorithm 9 on $\mathcal{P}_{v} \backslash\left\{v_{1}\right\}, R\left(\sigma\left(v_{3}\right), \sigma^{\prime}\left(v_{3}\right)\right), R\left(\sigma\left(v_{4}\right), \sigma^{\prime}\left(v_{4}\right)\right), \ldots$, $R\left(\sigma\left(v_{V-1}\right), \sigma^{\prime}\left(v_{V-1}\right)\right)$, and $\overline{p\left(v_{2}\right) v}$;

17:

\section{end if}

18: end if

19: if $U=2$ and $V=2$ then

20: $\quad p\left(z_{1}\right) \leftarrow$ point of $\operatorname{int}\left(R\left(\sigma\left(z_{1}\right), \sigma^{\prime}\left(z_{1}\right)\right)\right)$; place $z_{1}$ at $p\left(z_{1}\right)$;

21: end if

22: apply Algorithm 9 on $\mathcal{P}_{z}, R\left(\sigma\left(z_{2}\right), \sigma^{\prime}\left(z_{2}\right)\right), R\left(\sigma\left(z_{3}\right), \sigma^{\prime}\left(z_{3}\right)\right), \ldots$, $R\left(\sigma\left(z_{Z-1}\right), \sigma^{\prime}\left(z_{Z-1}\right)\right)$, and $\overline{p\left(z_{1}\right) z}$

23: draw each edge of $G$ between two vertices belonging to $\mathcal{P}_{u}$, to $\mathcal{P}_{v}$, or to $\mathcal{P}_{z}$ as a straight-line segment;

24: apply Algorithm 2 on each resulting linearly-ordered outerclustered graph completing a drawing of the corresponding outer face;

25: $\Gamma(C) \leftarrow$ the resulting internally-convex-separated drawing of $C$;

26: return $\Gamma(C)$; 
We prove that $\Gamma\left(\mathcal{C}_{u, v}, T_{u, v}\right)$ satisfies Property CS1 of Definition 3. The angles $\widehat{u_{2} u v}$ and $\widehat{v_{2} v u}$ incident to $u$ and $v$ inside $P_{u, v}$ are strictly less than $180^{\circ}$, since they are respectively less than angles $\widehat{z u v}$ and $\widehat{z v u}$, that are angles of $P$, which is a triangle. By construction, $v_{2}$ is contained inside triangle $T\left(p\left(u_{2}\right), v, p\left(z_{1}\right)\right)$, hence $\widehat{u_{2} v_{2} v}$ is the angle of a triangle having $u_{2}, v_{2}$, and $v$ as vertices, hence it is less than $180^{\circ}$. Finally, angle $\widehat{u u_{2} v_{2}}$ is less than $180^{\circ}$, since by construction $v_{2}$ is placed in the halfplane $H\left(l\left(u, p\left(u_{2}\right)\right), v\right)$ delimited by the line through $u$ and $p\left(u_{2}\right)$, and containing $v$.

We prove that $\Gamma\left(\mathcal{C}_{u, v}, T_{u, v}\right)$ satisfies Property CS2. Observe that $\sigma(u)$ and $\sigma(v)$ are the first and the last cluster in $\Sigma$, respectively, and that the angles $\widehat{u_{2} u v}$ and $\widehat{v_{2} v u}$ incident to $u$ and $v$ inside $P_{u, v}$ are strictly less than $180^{\circ}$, as proved above.

We prove that $\Gamma\left(\mathcal{C}_{u, v}, T_{u, v}\right)$ satisfies Property CS3. The existence of regions $R(\mu, v)$ inside $P_{u, v}$ descends from the existence of regions $R(\mu, v)$ inside $P$, where $v$ is any child of $\mu$ in $T_{u, v}$. Namely, the drawn edges cut each convex region $R(\mu, v)$ into two or three convex regions. Such regions satisfy the properties that have to be satisfied by $R(\mu, v)$ inside $P_{u, v}$, as can be easily deduced from the fact that the same properties are satisfied by $R(\mu, \nu)$ inside $P$.

Graphs $C_{u, v}, C_{u, z}$, and $C_{v, z}$ are, in general, not triconnected since there could exist chords: (i) in $\mathcal{C}_{u, v}$ between any vertex in $\mathcal{P}_{u} \backslash\left\{u_{1}\right\}$ and any vertex in $\mathcal{P}_{v} \backslash\left\{v_{1}\right\}$; (ii) in $\mathcal{C}_{u, z}$ between vertex $u_{1}$ and any vertex in $\mathcal{P}_{u} \backslash\left\{u_{1}\right\}$, and between any vertex in $\mathcal{P}_{u} \backslash\left\{u_{1}\right\}$ and any vertex in $\mathcal{P}_{z} \backslash\left\{z_{1}\right\}$; (iii) in $\mathcal{C}_{v, z}$ between vertex $v_{1}$ and any vertex in $\mathcal{P}_{v} \backslash\left\{v_{1}\right\}$, and between any vertex in $\mathcal{P}_{v} \backslash\left\{v_{1}\right\}$ and any vertex in $\mathcal{P}_{z} \backslash\left\{z_{1}\right\}$. By Lemma 2, each of such chords splits a linearly-ordered outerclustered graph into two smaller linearly-ordered outerclustered graphs. Further, by construction the end-vertices of each of such chords are not collinear with any other vertex of the cycle. Hence, by Lemma 3, inserting the chords as straight-line segments into drawings $\Gamma\left(\mathcal{C}_{u, v}, T_{u, v}\right), \Gamma\left(\mathcal{C}_{u, z}, T_{u, z}\right)$, and $\Gamma\left(\mathcal{C}_{v, z}, T_{v, z}\right)$, that are convex-separated by Lemma 15 , splits them into convex-separated drawings. When all the chords have been added, the underlying graphs of the resulting clustered graphs are all triconnected and internally-triangulated. Hence, Theorem 1 applies and an internallyconvex-separated drawing of each of such linearly-ordered outerclustered graphs can be constructed inside the corresponding outer face, thus obtaining an internallyconvex-separated drawing of $C$.

Now suppose that Condition 2 of Lemma 12 holds.

By Lemma 14, $C_{u, v}\left(G_{u, v}, T_{u, v}\right), C_{u, z}\left(G_{u, z}, T_{u, z}\right)$, and $C_{v, z}\left(G_{v, z}, T_{v, z}\right)$ are linearly-ordered outerclustered graphs.

Refer to Fig. 29. Suppose that $U>2$ and that $V>2$. Consider the unique child $\sigma^{\prime}\left(u_{i}\right)$ of $\sigma\left(u_{i}\right)$, for each $2 \leq i \leq U-1$. Notice that one of the two polygonal lines obtained as intersection of the triangle representing $(u, v, z)$ and $R\left(\sigma\left(u_{2}\right), \sigma^{\prime}\left(u_{2}\right)\right)$ lies on edge $(u, z)$. Consider a point $p\left(u_{2}\right)$ of $\operatorname{int}\left(R\left(\sigma\left(u_{2}\right), \sigma^{\prime}\left(u_{2}\right)\right)\right)$ arbitrarily close to $(u, z)$. Place $u_{2}$ at $p\left(u_{2}\right)$. Draw a straight-line segment $\overline{p\left(u_{2}\right) u}$ and place $u_{i}$ at any point of the segment $\operatorname{int}\left(R\left(\sigma\left(u_{i}\right), \sigma^{\prime}\left(u_{i}\right)\right)\right) \cap \overline{p\left(u_{2}\right) u}$, for each $3 \leq i \leq U-1$.

Denote by $T\left(p\left(u_{2}\right), v, z\right)$ the triangle having $p\left(u_{2}\right), v$, and $z$ as vertices. Denote also by $H\left(l\left(u, p\left(u_{2}\right)\right), v\right)$ the open half-plane delimited by the line through $u$ and $p\left(u_{2}\right)$, and containing $v$.

Let $\sigma^{\prime}\left(v_{i}\right)$ be the unique child of $\sigma\left(v_{i}\right)$, for each $2 \leq i \leq V-1$. Consider any point $p\left(v_{2}\right)$ in $H\left(l\left(u, p\left(u_{2}\right)\right), v\right) \cap \operatorname{int}\left(T\left(p\left(u_{2}\right), v, z\right)\right) \cap \operatorname{int}\left(R\left(\sigma\left(v_{2}\right), \sigma^{\prime}\left(v_{2}\right)\right)\right)$ and 


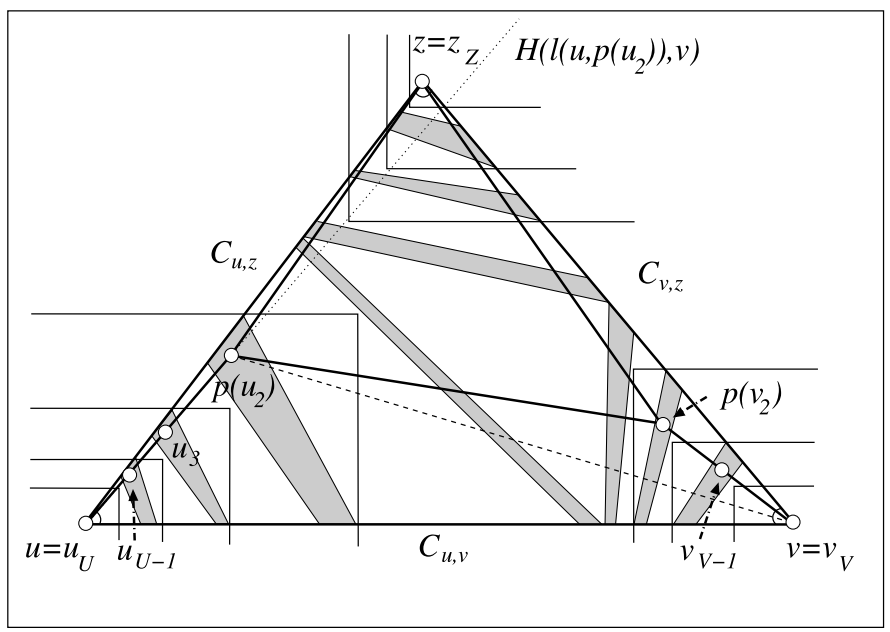

Fig. 29 Construction of drawings $\Gamma\left(\mathcal{C}_{u, v}, T_{u, v}\right), \Gamma\left(\mathcal{C}_{u, z}, T_{u, z}\right)$, and $\Gamma\left(\mathcal{C}_{v, z}, T_{v, z}\right)$ when Condition 2 of Lemma 12 holds

place $v_{2}$ at $p\left(v_{2}\right)$. Draw a straight-line segment $\overline{p\left(v_{2}\right) v}$ and place $v_{i}$ at any point of the segment $\operatorname{int}\left(R\left(\sigma\left(v_{i}\right), \sigma^{\prime}\left(v_{i}\right)\right)\right) \cap \overline{p\left(v_{2}\right) v}$, for each $3 \leq i \leq V-1$. Straightforward modifications make the described algorithm work also for the cases in which $U=2$ or $V=2$ (notice that $U=2$ and $V=2$ do not hold simultaneously when Condition 2 of Lemma 12 holds, otherwise $G$ would not have internal vertices). Such modifications are described in Algorithm 8 below.

Denote by $\Gamma\left(\mathcal{C}_{u, v}, T_{u, v}\right)$, by $\Gamma\left(\mathcal{C}_{u, z}, T_{u, z}\right)$, and by $\Gamma\left(\mathcal{C}_{v, z}, T_{v, z}\right)$ the constructed drawings of $\left(\mathcal{C}_{u, v}, T_{u, v}\right)$, of $\left(\mathcal{C}_{u, z}, T_{u, z}\right)$, and of $\left(\mathcal{C}_{v, z}, T_{v, z}\right)$, respectively. We have the following lemma, whose proof is analogous to the proof of Lemma 15.

Lemma $16 \Gamma\left(\mathcal{C}_{u, v}, T_{u, v}\right), \Gamma\left(\mathcal{C}_{u, z}, T_{u, z}\right)$, and $\Gamma\left(\mathcal{C}_{v, z}, T_{v, z}\right)$ are convex-separated drawings of the outer faces of $C_{u, v}\left(G_{u, v}, T_{u, v}\right), C_{u, z}\left(G_{u, z}, T_{u, z}\right)$, and $C_{v, z}\left(G_{v, z}\right.$, $\left.T_{v, z}\right)$, respectively.

Graphs $C_{u, v}, C_{u, z}$, and $C_{v, z}$ are, in general, not triconnected, since there could exist chords: (i) in $\mathcal{C}_{u, v}$ between any vertex in $\mathcal{P}_{u} \backslash\left\{u_{1}\right\}$ and any vertex in $\mathcal{P}_{v} \backslash\left\{v_{1}\right\}$; (ii) in $\mathcal{C}_{u, z}$ between vertex $u_{1}$ and any vertex in $\mathcal{P}_{u} \backslash\left\{u_{1}\right\}$; (iii) in $\mathcal{C}_{v, z}$ between vertex $v_{1}$ and any vertex in $\mathcal{P}_{v} \backslash\left\{v_{1}\right\}$. By Lemma 2, each of such chords splits a linearlyordered outerclustered graph into two smaller linearly-ordered outerclustered graphs. Further, by construction, the end-vertices of each of such chords are not collinear with any other vertex of the cycle. Hence, by Lemma 3, inserting the chords as straightline segments into drawings $\Gamma\left(\mathcal{C}_{u, v}, T_{u, v}\right), \Gamma\left(\mathcal{C}_{u, z}, T_{u, z}\right)$, and $\Gamma\left(\mathcal{C}_{v, z}, T_{v, z}\right)$, that are convex-separated by Lemma 16 , splits them into convex-separated drawings. When all chords have been added, the underlying graphs of the resulting clustered graphs are all triconnected and internally-triangulated. Hence, Theorem 1 applies and an internally-convex-separated drawing of each of such linearly-ordered outerclustered graphs can be constructed inside the corresponding outer face, thus obtaining an internally-convex-separated drawing of $C$. 
$\overline{\text { Algorithm } 8 \text { DraWing Maximal Outerclustered Graphs With TWO }}$ PATHS

Input: $C, \mathcal{P}_{u}=\left(u_{1}, \ldots, u_{U}\right), \sigma^{\prime}\left(u_{2}\right), \ldots, \sigma^{\prime}\left(u_{U-1}\right), \mathcal{P}_{v}=\left(v_{1}, \ldots, v_{V}\right), \sigma^{\prime}\left(v_{2}\right)$, $\ldots, \sigma^{\prime}\left(v_{V-1}\right)$, and $\Gamma\left(C_{o}\right)$;

Output: An internally-convex-separated drawing $\Gamma(C)$ of $C$ completing $\Gamma\left(C_{o}\right)$.

1: if $U>2$ then

2: $\quad p\left(u_{2}\right) \leftarrow$ point of $\operatorname{int}\left(R\left(\sigma\left(u_{2}\right), \sigma^{\prime}\left(u_{2}\right)\right)\right)$ close to $(u, z)$; place $u_{2}$ at $p\left(u_{2}\right)$;

3: $\quad$ apply Algorithm 9 on $\mathcal{P}_{u} \backslash\left\{u_{1}\right\}, R\left(\sigma\left(u_{3}\right), \sigma^{\prime}\left(u_{3}\right)\right), R\left(\sigma\left(u_{4}\right), \sigma^{\prime}\left(u_{4}\right)\right), \ldots$, $R\left(\sigma\left(u_{U-1}\right), \sigma^{\prime}\left(u_{U-1}\right)\right)$, and $\overline{p\left(u_{2}\right) u}$;

4: $\quad$ if $V>2$ then

5: $\quad H\left(l\left(u, p\left(u_{2}\right)\right), v\right) \leftarrow$ the open half-plane delimited by the line through $u$ and $p\left(u_{2}\right)$, and containing $v$;

6: $\quad p\left(v_{2}\right) \leftarrow$ point of $H\left(l\left(u, p\left(u_{2}\right)\right), v\right) \cap \operatorname{int}\left(T\left(p\left(u_{2}\right), v, z\right)\right) \cap$ $\operatorname{int}\left(R\left(\sigma\left(v_{2}\right), \sigma^{\prime}\left(v_{2}\right)\right)\right)$; place $v_{2}$ at $p\left(v_{2}\right)$;

7: $\quad$ apply Algorithm 9 on $\mathcal{P}_{v} \backslash\left\{v_{1}\right\}, R\left(\sigma\left(v_{3}\right), \sigma^{\prime}\left(v_{3}\right)\right), R\left(\sigma\left(v_{4}\right), \sigma^{\prime}\left(v_{4}\right)\right), \ldots$, $R\left(\sigma\left(v_{V-1}\right), \sigma^{\prime}\left(v_{V-1}\right)\right)$, and $\overline{p\left(v_{2}\right) v}$;

\section{8: $\quad$ end if}

9: else

10: $\quad p\left(v_{2}\right) \leftarrow$ point of $\operatorname{int}\left(R\left(\sigma\left(v_{2}\right), \sigma^{\prime}\left(v_{2}\right)\right)\right)$; place $v_{2}$ at $p\left(v_{2}\right)$;

11: apply Algorithm 9 on $\mathcal{P}_{v} \backslash\left\{v_{1}\right\}, R\left(\sigma\left(v_{3}\right), \sigma^{\prime}\left(v_{3}\right)\right), R\left(\sigma\left(v_{4}\right), \sigma^{\prime}\left(v_{4}\right)\right), \ldots$, $R\left(\sigma\left(v_{V-1}\right), \sigma^{\prime}\left(v_{V-1}\right)\right)$, and $\overline{p\left(v_{2}\right) v}$;

12: end if

13: draw each edge of $G$ between two vertices belonging to $\mathcal{P}_{u}$ or to $\mathcal{P}_{v}$ as a straightline segment;

14: apply Algorithm 2 on each resulting linearly-ordered outerclustered graph completing a drawing of the corresponding outer face;

15: $\Gamma(C) \leftarrow$ the resulting internally-convex-separated drawing of $C$;

16: return $\Gamma(C)$;

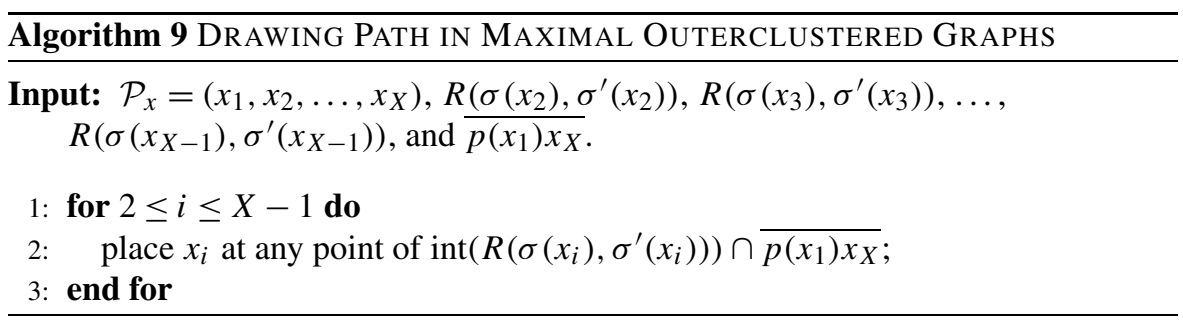

A pseudo-code description of the algorithm for drawing a maximal outerclustered graph $C$ (supposing that, if there exists a cluster containing exactly two vertices incident to $o(G)$, then such vertices are $u$ and $v$, and that, if the smallest containing cluster of one of $u$ and $v$ contains the other one, then $\sigma(v)$ contains $u$ ) is presented in Algorithms 6-9. 
Fig. 30 Illustration for Case 1 of the algorithm for drawing clustered graphs

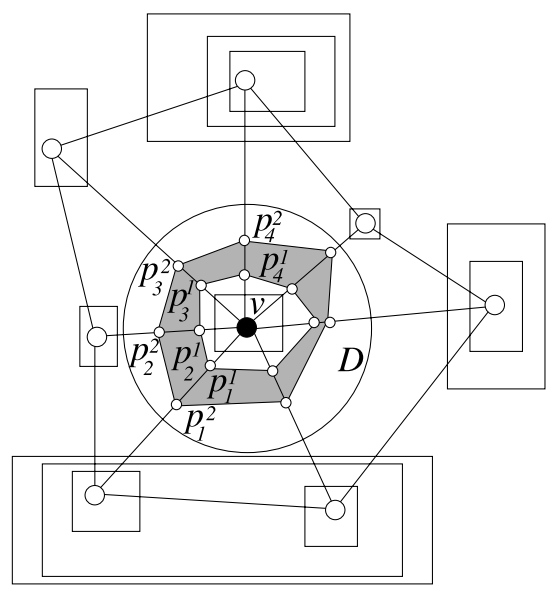

\section{Drawing Clustered Graphs}

In this section we prove that every clustered graph $C(G, T)$ admits an internallyconvex-separated drawing $\Gamma(C)$ completing an arbitrary triangular-convex-separated drawing $\Gamma\left(C_{o}\right)$ of $C_{o}$. Recall that a drawing $\Gamma\left(C_{o}\right)$ of $C_{o}$ consists of a drawing of the outer face $o(G)$ of $G$ and of a drawing of each cluster containing a vertex incident to $o(G)$. We assume that $\Gamma\left(C_{o}\right)$ is any triangular-convex-separated drawing $\Gamma\left(C_{o}\right)$ of $C_{o}$ and we show how to compute a straight-line rectangular drawing of $C$ completing $\Gamma\left(C_{o}\right)$. Such a result is achieved by means of an inductive algorithm, where the induction is on the number of vertices plus the number of clusters of $C$. In the base case, $C$ is a maximal outerclustered graph and the statement follows from Theorem 2. Consider any maximal clustered graph $C(G, T)$.

Case 1: There exists a minimal cluster containing exactly one internal vertex and no external vertex. Refer to Fig. 30. Let $\mu$ be a minimal cluster containing exactly one vertex $v$ internal to $G$ and containing no vertex incident to $o(G)$. Remove $\mu$ from $T$ obtaining a clustered graph $C^{\prime}\left(G, T^{\prime}\right)$. Observe that $C_{o}$ and $C_{o}^{\prime}$ are the same graph, since $\mu$ does not contain vertices incident to $o(G)$. The number of vertices plus the number of clusters of $C^{\prime}$ is one less than the number of vertices plus the number of clusters of $C$. Hence, the inductive hypothesis applies and, for an arbitrary triangularconvex-separated drawing $\Gamma\left(C_{o}\right)$, there exists an internally-convex-separated drawing $\Gamma\left(C^{\prime}\right)$ of $C^{\prime}$ completing $\Gamma\left(C_{o}\right)$. In $\Gamma\left(C^{\prime}\right)$ a small disk $D$ can be drawn with the following properties: it is centered at $v$, it does not intersect the boundary of any cluster, it does not contain any vertex of $G$ different from $v$, and it has intersection only with the edges incident to $v$. For each edge $e_{i}$ incident to $v$, choose two points $p_{i}^{1}$ and $p_{i}^{2}$ inside $D$, where $p_{i}^{1}$ is closer to $v$ than $p_{i}^{2}$. For each two edges $e_{i}$ and $e_{i+1}$ consecutively incident to $v$ denote by $f_{i}$ the face of $G$ incident to edges $e_{i}$ and $e_{i+1}$, and denote by $R(\mu, i)$ the quadrilateral having $p_{i}^{1}, p_{i}^{2}, p_{i+1}^{1}$, and $p_{i+1}^{2}$ as vertices. Finally, insert a drawing of $\mu$ in $\Gamma\left(C^{\prime}\right)$ as a rectangle containing $v$ and contained inside the polygon $\left(p_{1}^{1}, p_{2}^{1}, \ldots, p_{k}^{1}, p_{1}^{1}\right)$, thus obtaining a drawing $\Gamma(C)$.

Lemma $17 \Gamma(C)$ is an internally-convex-separated drawing of $C$. 
Proof $\Gamma(C)$ has no edge crossing, by induction. $\Gamma(C)$ has no edge-region crossing since any cluster different from $\mu$ has no crossing with any edge by induction, and since $\mu$ does not intersect any edge not incident to $v$, because it completely lies inside $D . \Gamma(C)$ has no region-region crossing since the boundaries of any two clusters different from $\mu$ have no crossing by induction, and since the boundary of $\mu$ does not intersect the boundary of any cluster, because it completely lies inside $D$. The drawing is straight-line and rectangular by construction. Further, every internal face of $G$ not incident to $v$ is triangular-convex-separated by induction. Finally, for each face $f_{i}$, define region $R(\nu, \mu)$, where $v$ is the parent of $\mu$ in the inclusion tree $T$ of $C(G, T)$, as coincident with region $R(\mu, i)$. Then, $R(\nu, \mu)$ satisfies Property TCS1 of a triangular-convex-separated drawing, due to the fact that such a region is completely contained inside $D$, and that $D$ is completely contained inside $v$.

Case 2: There exists a separating 3-cycle. Suppose that $G$ contains a separating 3-cycle $\left(u^{\prime}, v^{\prime}, z^{\prime}\right)$. Let $C^{1}\left(G^{1}, T^{1}\right)$ be the clustered graph defined as follows. $G^{1}$ is the subgraph of $G$ induced by all the vertices external to $\left(u^{\prime}, v^{\prime}, z^{\prime}\right)$, by $u^{\prime}$, by $v^{\prime}$, and by $z^{\prime} . T^{1}$ is the subtree of $T$ whose clusters contain at least one vertex of $G^{1}$. Observe that $C_{o}$ and $C_{o}^{1}$ are the same graph. Further, let $C^{2}\left(G^{2}, T^{2}\right)$ be the clustered graph defined as follows. $G^{2}$ is the subgraph of $G$ induced by all the vertices internal to $\left(u^{\prime}, v^{\prime}, z^{\prime}\right)$, by $u^{\prime}$, by $v^{\prime}$, and by $z^{\prime} . T^{2}$ is the subtree of $T$ whose clusters contain at least one vertex of $G^{2}$. Since $\left(u^{\prime}, v^{\prime}, z^{\prime}\right)$ is a separating 3 -cycle, the number of vertices plus the number of clusters of each of $C^{1}$ and $C^{2}$ is strictly less than the number of vertices plus the number of clusters of $C$. Hence, the inductive hypothesis applies and, for an arbitrary triangular-convex-separated drawing $\Gamma\left(C_{o}\right)$, there exists an internally-convex-separated drawing $\Gamma\left(C^{1}\right)$ of $C^{1}$ completing $\Gamma\left(C_{o}\right)$. Cycle $\left(u^{\prime}, v^{\prime}, z^{\prime}\right)$ is a face $f$ of $G^{1}$. By definition of internally-convex-separated drawing of a graph, the drawing $\Gamma\left(C_{f}\right)$ of $C_{f}$ in $\Gamma\left(C^{1}\right)$ is a triangular-convex-separated drawing. Observe that $C_{f}$ and $C_{o}^{2}$ are the same graph. Hence, the inductive hypothesis applies again and an internally-convex-separated drawing $\Gamma\left(C^{2}\right)$ can be constructed completing $\Gamma\left(C_{o}^{2}\right)$. Plugging $\Gamma\left(C^{2}\right)$ into $\Gamma\left(C^{1}\right)$ provides a drawing $\Gamma(C)$ of $C$.

\section{Lemma $18 \Gamma(C)$ is an internally-convex-separated drawing of $C$.}

Proof $\Gamma(C)$ has no edge crossing. Namely, any edge belonging to $G^{1}$ (resp. to $G^{2}$ ) does not cross any edge belonging to $G^{1}$ (resp. to $G^{2}$ ) by induction. Further, any edge belonging to $G^{1}$ and not belonging to $G^{2}$ does not cross any edge belonging to $G^{2}$ and not belonging to $G^{1}$ since such edges are separated by cycle $\left(u^{\prime}, v^{\prime}, z^{\prime}\right) . \Gamma(C)$ has no edge-region crossing. Namely, any edge belonging to $G^{1}$ (resp. to $G^{2}$ ) does not cross the boundary of any cluster of $T^{1}$ (resp. of $T^{2}$ ) by induction. Further, any edge belonging to $G^{1}$ (resp. to $G_{2}$ ) and not belonging to $G^{2}$ (resp. to $G^{1}$ ) does not cross the boundary of any cluster belonging to $T^{2}$ (resp. to $T^{1}$ ) and not belonging to $T^{1}$ (resp. to $T^{2}$ ), since such an edge and such a cluster are separated by cycle $\left(u^{\prime}, v^{\prime}, z^{\prime}\right) . \Gamma(C)$ has no region-region crossing. Namely, the boundary of any cluster belonging to $T^{1}$ (resp. to $T^{2}$ ) does not cross the boundary of any cluster belonging to $T^{1}$ (resp. to $T^{2}$ ) by induction. Further, the boundary of any cluster belonging to $T^{1}$ (resp. to $T^{2}$ ) and not belonging to $T^{2}$ (resp. to $T^{1}$ ) does not cross the boundary of 

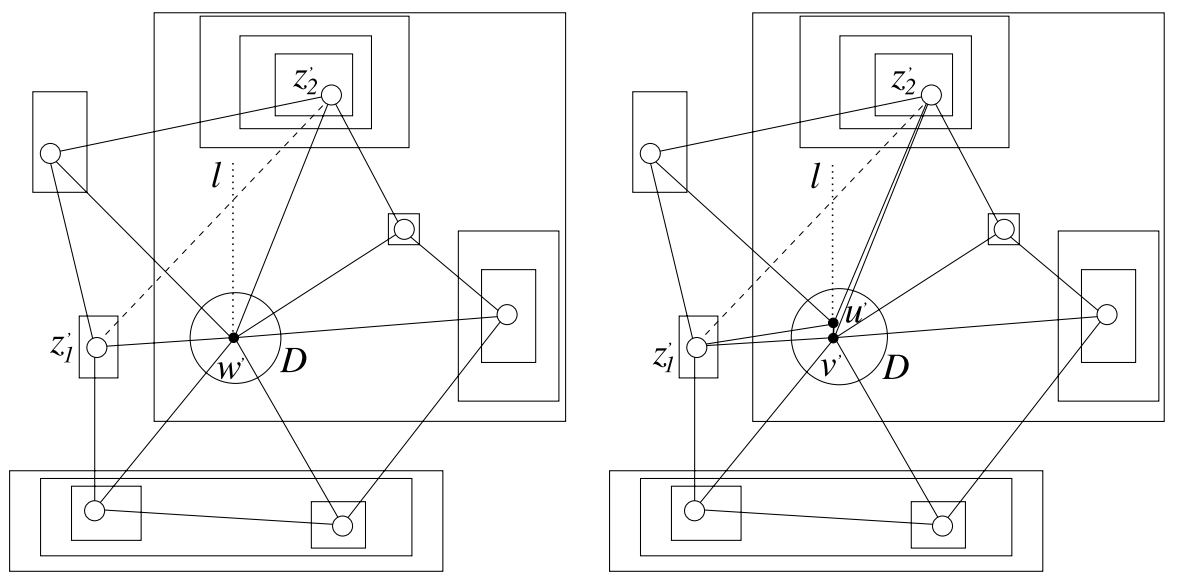

Fig. 31 Illustration for Case 3 of the algorithm for drawing clustered graphs

any cluster belonging to $T^{2}$ (resp. to $T^{1}$ ) and not belonging to $T^{1}$ (resp. to $T^{2}$ ), since such clusters are separated by cycle $\left(u^{\prime}, v^{\prime}, z^{\prime}\right) . \Gamma(C)$ is straight-line and rectangular by construction. Further, the drawing of any internal face $f$ of $G$ is triangular-convexseparated since it is triangular-convex-separated in $\Gamma\left(C^{1}\right)$ (if $f$ is also a face of $G^{1}$ ) or in $\Gamma\left(C^{2}\right)$ (if $f$ is also a face of $G^{2}$ ).

Case 3: There exists no separating 3-cycle and there exists an edge $\left(u^{\prime}, v^{\prime}\right)$ not incident to $o(G)$ such that $\sigma\left(u^{\prime}\right)=\sigma\left(v^{\prime}\right)$. Refer to Fig. 31. Suppose that $G$ contains an edge $\left(u^{\prime}, v^{\prime}\right)$ with $\sigma\left(u^{\prime}\right)=\sigma\left(v^{\prime}\right)$ such that $u^{\prime}$ is internal and suppose that there exists no separating 3-cycle containing edge $\left(u^{\prime}, v^{\prime}\right)$. Since $G$ is internally-triangulated, $u^{\prime}$ and $v^{\prime}$ have exactly two common neighbors $z_{1}^{\prime}$ and $z_{2}^{\prime}$. Contract edge $\left(u^{\prime}, v^{\prime}\right)$ to a vertex $w^{\prime}$, that is, replace vertices $u^{\prime}$ and $v^{\prime}$ with a single vertex $w^{\prime}$ that is connected to all the vertices $u^{\prime}$ and $v^{\prime}$ are connected to. Vertex $w^{\prime}$ belongs to cluster $\sigma\left(u^{\prime}\right)$ and to all the ancestors of $\sigma\left(u^{\prime}\right)$ in $T$. The resulting clustered graph $C^{\prime}\left(G^{\prime}, T^{\prime}\right)$ is easily shown to be a maximal $c$-planar clustered graph. In particular, the absence of separating 3-cycles in $G$ guarantees that $G^{\prime}$ is simple and internally-triangulated. Observe that $C_{o}$ and $C_{o}^{\prime}$ are the same graph. Hence, the inductive hypothesis applies and, for an arbitrary triangular-convex-separated drawing $\Gamma\left(C_{o}\right)$, there exists an internallyconvex-separated drawing $\Gamma\left(C^{\prime}\right)$ of $C^{\prime}$ completing $\Gamma\left(C_{o}\right)$. Then, consider a small disk $D$ centered at $w^{\prime}$ and consider any line $l$ from $w^{\prime}$ to an interior point of the segment between $z_{1}^{\prime}$ and $z_{2}^{\prime}$. Replace $w^{\prime}$ with $u^{\prime}$ and $v^{\prime}$ so that such vertices lie on $l$ and inside $D$. Connect $u^{\prime}$ and $v^{\prime}$ to their neighbors, obtaining a drawing $\Gamma(C)$ of $C$.

Lemma $19 \Gamma(C)$ is an internally-convex-separated drawing of $C$.

Proof In [14] the following property of planar straight-line drawings has been proved. In a planar straight-line drawing, for any vertex $w^{\prime}$, there exists a disk $D$ centered at $w^{\prime}$ such that moving $w^{\prime}$ to any point inside $D$ leaves the straight-line drawing planar. The proof of this property takes into account the set of points from 
which all the neighbors of $w^{\prime}$ are visible, i.e., straight-line segments can be drawn without causing crossings. This property of planar straight-line drawings has been exploited in $[9,10,14]$ to argue that, in a graph $G$ with no separating 3-cycle, an edge $\left(u^{\prime}, v^{\prime}\right)$ that has been contracted to a single vertex $w^{\prime}$ (obtaining a graph $G^{\prime}$ ) can suitably replace $w^{\prime}$ so that the resulting straight-line drawing of $G$ is planar. Here the continuity arguments used in [14] to prove the existence of $D$ are still valid; however, the visibility between any point $p$ of $D$ and any neighbor $z^{\prime}$ of $w^{\prime}$ means that it is possible to draw a straight-line segment from $p$ to $z^{\prime}$ not crossing any edge of $G$ and not crossing twice the boundary of the same cluster; further, $D$ has to be so small that it does not intersect the boundary of any cluster.

Then, the placement of $u^{\prime}$ and $v^{\prime}$ guarantees that $\Gamma(C)$ has no edge crossing and no edge-region crossing. Further, $\Gamma(C)$ has no region-region crossing, by induction. $\Gamma(C)$ is a straight-line rectangular drawing, by construction. Finally, for each internal face $f$ of $G$ not incident to $u^{\prime}$ and $v^{\prime}$ regions $R(\mu, v)$ can be drawn as in $\Gamma\left(C^{\prime}\right)$, since $f$ has the same drawing in $\Gamma(C)$ and in $\Gamma\left(C^{\prime}\right)$; for each internal face $f$ of $G$ incident to $u^{\prime}$ and not to $v^{\prime}$, or vice versa, regions $R(\mu, v)$ can be drawn similarly to $\Gamma\left(C^{\prime}\right)$, since the drawings of $f$ in $\Gamma(C)$ and in $\Gamma\left(C^{\prime}\right)$ differ for an arbitrary small displacement of an incident vertex (in $C^{\prime}$, face $f$ is incident to vertex $w^{\prime}$, that replaces the one out of $u^{\prime}$ and $v^{\prime}$ that is incident to $f$ in $\left.C\right)$; faces $\left(u^{\prime}, v^{\prime}, z_{1}^{\prime}\right)$ and $\left(u^{\prime}, v^{\prime}, z_{2}^{\prime}\right)$ are so thin that no vertex of any rectangle representing a cluster lies inside such faces, hence regions $R(\mu, v)$ can easily be drawn.

It remains to prove that the case in which $C$ is a maximal outerclustered graph is the base case.

Lemma 20 Suppose that none of Cases 1, 2, and 3 applies. Then $C$ is a maximal outerclustered graph.

Proof That $G$ is a maximal plane graph is easily proved by the observation that each of Cases 1, 2, and 3 transforms a clustered graph whose underlying graph is a maximal plane graph into one or two clustered graphs whose underlying graphs are maximal plane graphs. We now prove that $C$ is an outerclustered graph.

Suppose, for a contradiction, that none of Cases 1, 2, and 3 applies, and that $C$ is not an outerclustered graph. By the maximality of $G, o(G)$ is delimited by a 3-cycle. By the $c$-planarity of $C$, each cluster that contains some but not all the vertices incident to $o(G)$ intersects $o(G)$ exactly twice, thus proving Property O2 of Definition 1 .

Suppose that $C$ contains a cluster $\mu$ not containing any vertex incident to $o(G)$. Then, $C$ contains a minimal cluster $\mu^{\prime}$ not containing any vertex incident to $o(G)$, namely $\mu^{\prime}=\mu$ if $\mu$ is minimal, and $\mu^{\prime}$ is any minimal cluster descendant of $\mu$, if $\mu$ is not minimal. If $\mu^{\prime}$ contains exactly one vertex $v$, then $\mu^{\prime}$ is a minimal cluster containing only $v$, and Case 1 applies. If $\mu^{\prime}$ contains more than one vertex, then, by the $c$-connectivity of $C$, there exists at least one edge $(u, v)$ such that $\sigma(u)=$ $\sigma(v)=\mu^{\prime}$. If $(u, v)$ is an edge of a separating 3-cycle, then Case 2 applies. Otherwise, Case 3 applies. This proves Property O1.

Suppose that $C$ contains an edge $(u, v)$ such that $\sigma(u)=\sigma(v)$ and suppose that at least one out of $u$ and $v$ is an internal vertex of $G$. If edge $(u, v)$ belongs to a 


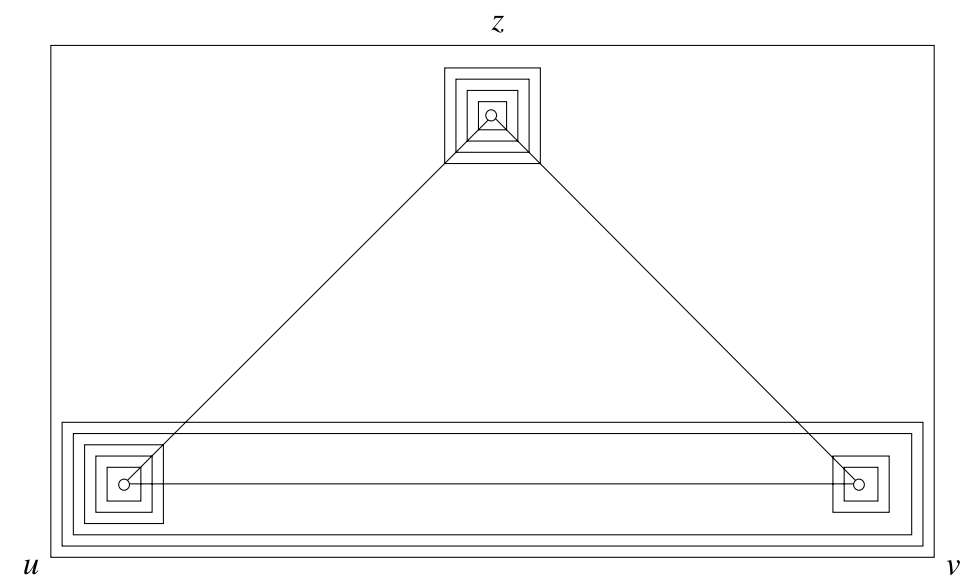

Fig. 32 The triangular-convex-separated drawing of the outer face of a clustered graph $C$ constructed by Algorithm 11

separating 3-cycle, then Case 2 applies. Otherwise, Case 3 applies. This proves Property $\mathrm{O} 3$.

Hence, we get the following:

Theorem 3 Let $C(G, T)$ be a maximal c-planar clustered graph. Then, for every triangular-convex-separated drawing $\Gamma\left(C_{o}\right)$ of $C_{o}$, there exists an internally-convexseparated drawing $\Gamma(C)$ of $C$ completing $\Gamma\left(C_{o}\right)$.

A pseudo-code description of the algorithm for drawing a clustered graph $C$, given an arbitrary triangular-convex-separated drawing $\Gamma\left(C_{o}\right)$ of $C_{o}$ is presented in Algorithm 10. A pseudo-code description of the algorithm for drawing a clustered graph $C$ is presented in Algorithm 11. An example of the triangular-convex-separated drawing of the outer face of a clustered graph $C$ constructed by Algorithm 11 is depicted in Fig. 32.

\section{Conclusions}

In this paper we have shown that every $c$-planar clustered graph admits a $c$-planar straight-line rectangular drawing. Actually, the algorithms we proposed do not exploit at all the fact that clusters are drawn as rectangles (except for the construction of a triangular-convex-separated drawing of the outer face of the input graph). Hence, such algorithms can be modified in order to construct a $c$-planar straight-line drawing of a given clustered graph for an arbitrary assignment of convex shapes to the clusters.

The algorithm we described in this paper uses real coordinates, hence it requires exponential area to be implemented in a system with a finite resolution rule. However, this drawback is unavoidable, since it has been proved by Feng et al. [16] that there 


\section{Algorithm 10 DraWing Clustered Graphs With Given Outer FaCe}

Input: A $c$-planar embedding of a clustered graph $C(G, T)$ and a triangular-convexseparated drawing $\Gamma\left(C_{o}\right)$ of $C_{o}$.

Output: An internally-convex-separated drawing $\Gamma(C)$ of $C$ completing $\Gamma\left(C_{o}\right)$.

1: if a minimal cluster $\mu$ exists containing exactly one internal vertex $v$ and no external vertex then

2: $\quad C^{\prime} \leftarrow$ the graph obtained from $C$ by removing $\mu$;

3: $\quad \Gamma\left(C^{\prime}\right) \leftarrow$ the drawing obtained by applying Algorithm 10 on $C^{\prime}$ and $\Gamma\left(C_{o}\right)$;

4: $\quad \Gamma(C) \leftarrow$ the drawing obtained by inserting $\mu$ in $\Gamma\left(C^{\prime}\right)$ as a small rectangle containing $v$;

5: else

6: $\quad$ if there exists a separating 3-cycle $f=\left(u^{\prime}, v^{\prime}, z^{\prime}\right)$ then

7: $\quad G^{1} \leftarrow$ the subgraph of $G$ induced by the vertices external to or belonging to $\left(u^{\prime}, v^{\prime}, z^{\prime}\right)$;

8: $\quad T^{1} \leftarrow$ the subtree of $T$ whose clusters contain vertices of $G^{1}$;

9: $\quad C^{1} \leftarrow\left(G^{1}, T^{1}\right)$;

10: $\quad G^{2} \leftarrow$ the subgraph of $G$ induced by the vertices internal to or belonging to $\left(u^{\prime}, v^{\prime}, z^{\prime}\right)$;

11: $\quad T^{2} \leftarrow$ the subtree of $T$ whose clusters contain vertices of $G^{2}$;

12: $\quad C^{2} \leftarrow\left(G^{2}, T^{2}\right)$;

13: $\quad \Gamma\left(C^{1}\right) \leftarrow$ the drawing obtained by applying Algorithm 10 on $C^{1}$ and $\Gamma\left(C_{o}\right)$;

14: $\quad \Gamma\left(C_{f}\right) \leftarrow$ the drawing of $C_{f}$ in $\Gamma\left(C^{1}\right)$;

15: $\quad \Gamma\left(C^{2}\right) \leftarrow$ the drawing obtained by applying Algorithm 10 on $C^{2}$ and $\Gamma\left(C_{f}\right)$;

16: $\quad \Gamma(C) \leftarrow$ the drawing obtained by plugging $\Gamma\left(C^{2}\right)$ into $\Gamma\left(C^{1}\right)$;

17: $\quad$ else

18: $\quad$ if an edge $\left(u^{\prime}, v^{\prime}\right)$ exists not incident to $o(G)$ such that $\sigma\left(u^{\prime}\right)=\sigma\left(v^{\prime}\right)$ then

$z_{1}^{\prime}$ and $z_{2}^{\prime} \leftarrow$ the common neighbors of $u^{\prime}$ and $v^{\prime}$;

$G^{\prime} \leftarrow$ the graph obtained from $G$ by contracting $\left(u^{\prime}, v^{\prime}\right)$ to a vertex $w^{\prime}$;

$T^{\prime} \leftarrow$ the tree obtained from $T$ by assigning $w^{\prime}$ to $\sigma\left(u^{\prime}\right)$;

$C^{\prime} \leftarrow\left(G^{\prime}, T^{\prime}\right)$

$\Gamma\left(C^{\prime}\right) \leftarrow$ the drawing obtained by applying Algorithm 10 on $C^{\prime}$ and $\Gamma\left(C_{o}\right)$;

24: $\quad l \leftarrow$ a line from $w^{\prime}$ to an interior point of $\overline{z_{1}^{\prime} z_{2}^{\prime}}$ in $\Gamma\left(C^{\prime}\right)$;

25: $\quad \quad \quad \quad C(C) \leftarrow$ the drawing obtained from $\Gamma\left(C^{\prime}\right)$ by replacing $w^{\prime}$ with $u^{\prime}$ and $v^{\prime}$ so that $u^{\prime}$ and $v^{\prime}$ lie on $l$ inside a small disk centered at $w^{\prime}$;

$\Gamma(C) \leftarrow$ the drawing obtained by applying Algorithm 6 on $C$ and $\Gamma\left(C_{o}\right)$; end if

31: return $\Gamma(C)$; 
Algorithm 11 DRAWING ClUSTERED GRAPHS

Input: A $c$-planar embedding of a clustered graph $C(G, T)$.

Output: A straight-line rectangular drawing $\Gamma(C)$ of $C$.

1: add dummy edges to $G$ until $C$ is maximal;

2: $u, v, z \leftarrow$ the vertices incident to $o(G)$;

3: if a cluster $\mu$ containing exactly two out of $u, v$, and $z$ exists then

4: $\quad u, v \leftarrow$ be the vertices out of $u, v$, and $z$ belonging to $\mu$;

5: end if

6: $\epsilon \leftarrow 1 / n^{2}$

7: Place $u$ at $(0,0)$; place $v$ at $(2,0)$; place $z$ at $(1,1)$;

8: $i, j \leftarrow 1$;

9: while a not drawn cluster exists containing $u$ and not containing $v$ do

10: $\quad \mu \leftarrow$ smallest cluster in $T$ containing $u$, not containing $v$, and not drawn;

11: Draw $\mu$ with corners $(i \epsilon, i \epsilon),(i \epsilon,-i \epsilon),(-i \epsilon,-i \epsilon),(-i \epsilon, i \epsilon)$;

12: $\quad i \leftarrow i+1$;

13: end while

14: while a not drawn cluster exists containing $v$ and not containing $u$ do

15: $\quad \mu \leftarrow$ smallest cluster in $T$ containing $v$, not containing $u$, and not drawn;

16: Draw $\mu$ with corners $(2+j \epsilon, j \epsilon),(2+j \epsilon,-j \epsilon),(2-j \epsilon,-j \epsilon),(2-j \epsilon, j \epsilon)$;

17: $\quad j \leftarrow j+1$;

18: end while

19: $k \leftarrow \max \{i, j\}$;

20: while a not drawn cluster exists containing $u$ and $v$ and not containing $z$ do

21: $\quad \mu \leftarrow$ smallest cluster in $T$ containing $u$ and $v$, not containing $z$, and not drawn;

22: Draw $\mu$ with corners $(2+k \epsilon, k \epsilon),(2+k \epsilon,-k \epsilon),(-k \epsilon,-k \epsilon),(-k \epsilon, k \epsilon)$;

23: $\quad k \leftarrow k+1$;

24: end while

25: $l \leftarrow 1$;

26: while a not drawn cluster exists containing $z$ and not containing $u$ and $v$ do

27: $\quad \mu \leftarrow$ smallest cluster in $T$ containing $z$, not containing $u$ and $v$, and not drawn;

28: Draw $\mu$ with corners $(1+l \epsilon, 1+l \epsilon),(1+l \epsilon, 1-l \epsilon),(1-l \epsilon, 1-l \epsilon),(1-$ $l \epsilon, 1+l \epsilon)$;

29: $\quad l \leftarrow l+1$;

30: end while

31: $m \leftarrow \max \{k, l\}$;

32: while a not drawn cluster exists containing $u, v$, and $z$ do

33: $\quad \mu \leftarrow$ smallest cluster in $T$ containing $u, v$, and $z$, and not drawn;

34: Draw $\mu$ with corners $(2+m \epsilon, 1+m \epsilon),(2+m \epsilon,-m \epsilon),(-m \epsilon,-m \epsilon)$, $(-m \epsilon, 1+m \epsilon)$;

35: $\quad m \leftarrow m+1$;

36: end while

37: $\Gamma\left(C_{o}\right) \leftarrow$ the resulting drawing of $C_{o}$;

38: $\Gamma(C) \leftarrow$ the drawing obtained by applying Algorithm 10 on $C$ and $\Gamma\left(C_{o}\right)$;

39: remove dummy edges from $\Gamma(C)$;

40: return $\Gamma(C)$; 
exist clustered graphs requiring exponential area in any straight-line drawing in which clusters are represented by convex regions.

\section{References}

1. Cornelsen, S., Wagner, D.: Completely connected clustered graphs. J. Discrete Algorithms 4(2), 313323 (2006)

2. Cortese, P.F., Di Battista, G., Frati, F., Patrignani, M., Pizzonia, M.: C-planarity of c-connected clustered graphs. J. Graph Algorithms Appl. 12(2), 225-262 (2008)

3. Cortese, P.F., Di Battista, G., Patrignani, M., Pizzonia, M.: Clustering cycles into cycles of clusters. J. Graph Algorithms Appl. 9(3), 391-413 (2005)

4. Cortese, P.F., Di Battista, G., Patrignani, M., Pizzonia, M.: On embedding a cycle in a plane graph. Discrete Math. 309(7), 1856-1869 (2009)

5. Dahlhaus, E.: A linear time algorithm to recognize clustered graphs and its parallelization. In: Lucchesi, C.L., Moura, A.V. (eds.) Latin American Symposium on Theoretical Informatics (LATIN '98), pp. 239-248 (1998)

6. Di Battista, G., Drovandi, G., Frati, F.: How to draw a clustered tree. J. Discrete Algorithms 7(4), 479-499 (2009)

7. Di Battista, G., Eades, P., Tamassia, R., Tollis, I.G.: Graph Drawing. Prentice Hall, Upper Saddle River (1999)

8. Di Battista, G., Frati, F.: Efficient c-planarity testing for embedded flat clustered graphs with small faces. J. Graph Algorithms Appl. 13(3), 349-378 (2009)

9. Di Battista, G., Frati, F., Patrignani, M.: Non-convex representations of graphs. In: Tollis, I., Patrignani, M. (eds.) Graph Drawing (GD ’08), pp. 390-395 (2008)

10. Di Battista, G., Tamassia, R.: Algorithms for plane representations of acyclic digraphs. Theor. Comput. Sci. 61, 175-198 (1988)

11. Eades, P., Feng, Q., Lin, X., Nagamochi, H.: Straight-line drawing algorithms for hierarchical graphs and clustered graphs. Algorithmica 44(1), 1-32 (2006)

12. Eades, P., Feng, Q., Nagamochi, H.: Drawing clustered graphs on an orthogonal grid. J. Graph Algorithms Appl. 3(4), 3-29 (1999)

13. Eades, P., Feng, Q.W., Lin, X.: Straight-line drawing algorithms for hierarchical graphs and clustered graphs. In: North, S. (ed.) Graph Drawing (GD ’96), pp. 113-128 (1996)

14. Fáry, I.: On straight line representations of planar graphs. Acta Sci. Math. 11, 229-233 (1948)

15. Feng, Q.: Algorithms for drawing clustered graphs. Ph.D. Thesis, The University of Newcastle, Australia (1997)

16. Feng, Q., Cohen, R.F., Eades, P.: How to draw a planar clustered graph. In: Du, D., Li, M. (eds.) Computing and Combinatorics (COCOON'95), pp. 21-30 (1995)

17. Feng, Q., Cohen, R.F., Eades, P.: Planarity for clustered graphs. In: Spirakis, P.G. (ed.) European Symposium on Algorithms (ESA '95), pp. 213-226 (1995)

18. Goodrich, M.T., Lueker, G.S., Sun, J.Z.: C-planarity of extrovert clustered graphs. In: Healy, P., Nikolov, N.S. (eds.) Graph Drawing (GD ’05), pp. 211-222 (2005)

19. Gutwenger, C., Jünger, M., Leipert, S., Mutzel, P., Percan, M., Weiskircher, R.: Advances in c-planarity testing of clustered graphs. In: Kobourov, S.G., Goodrich, M.T. (eds.) Graph Drawing (GD '02), pp. 220-235 (2002)

20. Jelínek, V., Jelínková, E., Kratochvíl, J., Lidický, B.: Clustered planarity: Embedded clustered graphs with two-component clusters. In: Tollis, I.G., Patrignani, M. (eds.) Graph Drawing (GD '08), pp. 121-132 (2008)

21. Jelínek, V., Suchý, O., Tesar, M., Vyskocil, T.: Clustered planarity: Clusters with few outgoing edges. In: Tollis, I.G., Patrignani, M. (eds.) Graph Drawing (GD '08), pp. 102-113 (2008)

22. Jelínková, E., Kára, J., Kratochvíl, J., Pergel, M., Suchý, O., Vyskocil, T.: Clustered planarity: Small clusters in cycles and eulerian graphs. J. Graph Algorithms Appl. 13(3), 379-422 (2009)

23. Jünger, M., Leipert, S., Percan, M.: Triangulating clustered graphs. Technical Report, Zentrum für Angewandte Informatik Köln, Lehrstuhl Jünger, December 2002

24. Kaufmann, M., Wagner, D. (eds.): Drawing Graphs, Methods and Models. Lecture Notes in Computer Science. Springer, Berlin (2001)

25. Nagamochi, H., Kuroya, K.: Drawing c-planar biconnected clustered graphs. Discrete Appl. Math. 155(9), 1155-1174 (2007) 